Sous la direction de claire Nguyen 


\title{
Mettre en œuvre un service de questions-réponses en ligne
}

\author{
Claire Nguyen (dir.)
}

DOI : 10.4000/books.pressesenssib.162

Éditeur : Presses de l'enssib

Année d'édition : 2010

Date de mise en ligne : 4 avril 2017

Collection : La Boîte à outils

ISBN électronique : 9782375460313

\section{QbOOOKS}

http://books.openedition.org

\section{Édition imprimée}

ISBN : 9782910227821

Nombre de pages : 210

\section{Référence électronique}

NGUYEN, Claire (dir.). Mettre en œuvre un service de questions-réponses en ligne. Nouvelle édition [en ligne]. Villeurbanne: Presses de l'enssib, 2010 (généré le 01 février 2021). Disponible sur Internet: <http://books.openedition.org/pressesenssib/162>. ISBN : 9782375460313. DOI : https://doi.org/ 10.4000/books.pressesenssib.162.

(c) Presses de l'enssib, 2010

Conditions d'utilisation

http://www.openedition.org/6540 


\section{\#20}

METTRE EN CEUVRE UN SERVICE DE QUESTIONS-RÉPONSES EN LIGNE

sous la direction de claire Nguyen 
METTRE EN CEUVRE

UN SERVICE DE

QUESTIONS-RÉPONSES

EN LIGNE 


\section{LA BOÎTE À OUTILS}

Les manuels de cette collection visent à fournir aux professionnels des ouvrages pratiques pour conduire des projets bibliothéconomiques d'actualité concernant aussi bien des bibliothèques publiques que des bibliothèques universitaires ou de recherche. Écrit à plusieurs mains, chaque volume est coordonné par un professionnel des bibliothèques.

\section{Catherine Jackson}

directrice de la collection
Déjà parus :

$+++++++++++++++++++++++++++++++++++++++$

BA0 \#16

Gérer les périodiques

sous la direction de Géraldine Barron (2008)

\section{BA0 \#17}

Favoriser la réussite des étudiants sous la direction de Carine El BekriDinoird (2009)

BA0 \#18

Mettre en œuvre un plan de classement sous la direction de Bertrand Calenge (2009)

\section{BA0 \#19}

Numériser et mettre en ligne sous la direction de Thierry Claerr et Isabelle Westeel (2010)

À paraître :

$+++++++++++++++++++++++++++++++++++++++$ BA0 \#21

Communiquer!

Les bibliothécaires, les décideurs et les journalistes sous la direction de Jean-Philippe Accart

\section{PRESSES DE L'enssib}

École nationale supérieure des sciences de l'information et des bibliothèques 17-21 boulevard du 11 novembre 1918 69623 Villeurbanne Cedex Tél. 0472444343 - Fax 0472444344 < http://www.enssib.fr/presses > 
METTRE EN CEUVRE UN SERVICE DE QUESTIONS-RÉPONSES EN LIGNE

Sous la direction de claire Nguyen 
Mes plus chaleureux remerciements vont à Catherine Jackson, directrice de la collection, qui a eu une écoute attentive et constructive et dont le soutien moral et amical sans faille a permis l'élaboration de cet ouvrage. Je remercie également Caroline Bruley, David Benoist, Nicolas Beudon, David Soret qui ont bien voulu relire mes propres contributions. Enfin, je suis vivement reconnaissante envers tous les auteurs, qui ont collaboré avec sérieux et empathie à leurs parties respectives mais aussi à toute l'architecture de l'ouvrage.

Claire Nguyen

Ont contribué à cet ouvrage :

$\begin{array}{ll}\text { Jean-Philippe Accart } & \begin{array}{l}\text { Pierre Moison } \\ \text { Marie Montano } \\ \text { Nicolas Alarcon } \\ \text { Caroline Bruley }\end{array} \\ \text { Bertrand Calenge } & \begin{array}{l}\text { Tù-Tâm Nguyen } \\ \text { Annie Prunet } \\ \text { Anabelle Copin }\end{array} \\ \text { Nathalie Daigne } & \text { Anna Svenbro } \\ \text { Marie-Françoise Defosse } & \text { Hélène Tardif } \\ \text { Christelle Di Pietro } & \\ \text { Hélène Gautier-Gentès } & \\ \text { Jean-Charles Houpier } & \\ \text { Nadine Kiker } & \\ \text { Sandrine Lebastard } & \\ \text { François Michaud } & \end{array}$

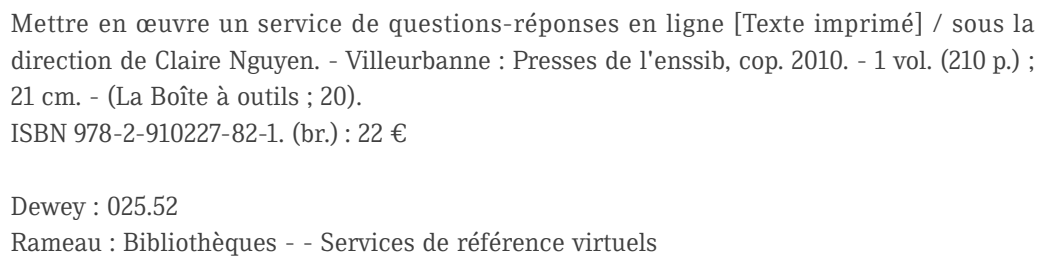

(c) enssib, 2010. 


\section{SOMMAIRE}

MODE D'EMPLOI

p. 9

par Claire Nguyen

PARTIE I : CONSTRUIRE LE PROJET .

p. 15

$+++++++++++++++++++++++++++++$

LA DÉMARCHE PROJET EN VUE DE LA CRÉATION D'UN SERVICE DE QUESTIONS-RÉPONSES EN LIGNE

par Jean-Philippe Accart p. 16

POLITIQUe DE SERVICE ET MODĖLES D'ORGANISATION

par Claire Nguyen p. 34
ENCADRÉS :

La politique de service en 9 points p. 34

Les modèles collaboratifs p. 45

LE RENSEIGNEMENT EN LIGNE AU SCD LYON 1 : LA DÉMARCHE PROJET

par Caroline Bruley

p. 46
COMMENT PROMOUVOIR UN SERVICE DE RENSEIGNEMENT EN LIGNE?

par Nicolas Alarcon p. 51

SERVICE DE QUESTIONSRÉPONSES ET ENVIRONNEMENT DE L'USAGER : QUELLE INTÉGRATION ? par Jean-Charles Houpier p. 59

VALORISER LE SERVICE DE QUESTIONS-RÉPONSES ET L'INTÉGRER AUX AUTRES SERVICES DE LA BIBLIOTHĖQUE par Bertrand Calenge p. 68

PARTIE II : RÉPONDRE : OUI, MAIS COMMENT ? ...

p. 77

COMMENT RÉPONDRE ET QU'EST-CE QUE BIEN RÉPONDRE ? CONSTRUIRE LE SAVOIR-RÉPONDRE par Marie-Françoise Defosse p. 78

OFFRIR UNE BASE DE CONNAISSANCES : POURQUOI, POUR QUI, COMMENT ?

par Marie Montano p. 89 
LE CADRE JURIDIQUE DES QUESTIONS ET DES RÉPONSES par Anna Svenbro p. 92

QUELS OUTILS POUR QUELS SERVICES ? QUELLE TECHNOLOGIE CHOISIR ? par Hélène Tardif p. 94

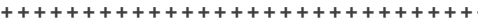
ENCADRÉ : Bibliothèques utilisant les logiciels présentés p. 109

\section{COMMENT ÉVALUER UN} SERVICE DE QUESTIONSRÉPONSES EN LIGNE ? par Nicolas Alarcon p. 110
PARTIE III : ORGANISER SON SERVICE p. 119

$++++++++++++++++++++++++++++++$

COMMENT S'ORGANISER EN INTERNE ; LE SAVOIR-ÊTRE DU RÉPONDEUR

par Sandrine Lebastard

et Tù-Tâm Nguyên p. 120

ENCADRÉ : 5 types de formations à mettre en œuvre p. 122
TRAVAILLER ENSEMBLE :

L'EXEMPLE D'UBIB.FR

par Nadine Kiker p. 130

RUE DES FACS

par François Michaud p. 137

LE SI@DE, UN LABEL ET UN RÉSEAU DE COOPÉRATION ENTRE SERVICES par Isabelle Copin p. 142
PARTIE IV : ADAPTER SON SERVICE À L'ENVIRONNEMENT ET À LA NATURE DE SES USAGERS ET DE SES COLLECTIONS p. 145

TYPOLOGIE DES SERVICES DE QUESTIONS-RÉPONSES

par Claire Nguyen p. 146

CONSTRUIRE UN SERVICE DE RÉFÉRENCE GÉNÉRALISTE : L'EXEMPLE DU GUICHET DU SAVOIR DE LA BIBLIOTHÈQUE MUNICIPALE DE LYON par Christelle Di Pietro p. 152

SINDBAD, LES PUBLICS ET LES COLLECTIONS DE LA BnF par Isabelle Copin p. 166 
DE BIBLIOSÉS@ME À BIBLIOSÉSAME.ORG : UN RÉSEAU GÉNÉRALISTE DE QUESTIONS-RÉPONSES DE BIBLIOTHĖQUES PUBLIQUES, PREMIER BILAN

par Nathalie Daigne p. 169
MÉMENTO

par Claire Nguyen ............................. p. 195

SIGLES ET ACRONYMES .... p. 201

GLOSSAIRE

BIBLIOGRAPHIE p. 205

LISTE DES AUTEURS

VOUS AVEZ DES QUESTIONS?

LES BIBLIOTHÉCAIRES DU RÉSEAU BIBLIOSÉSAME VOUS RÉPONDENT !

L'EXEMPLE DE LA BMVR DE MARSEILLE

par Annie Prunet p. 174

UN SERVICE DE QUESTIONSRÉPONSES EN LIGNE DE BIBLIOTHĖQUE SPÉCIALISÉE : BIUMINFO, LE SERVICE DE LA BIBLIOTHĖQUE INTERUNIVERSITAIRE DE MÉDECINE ET D'ODONTOLOGIE par Hélène Gautier-Gentès p. 177

QUESTIONS-SANTÉ, LE SERVICE DE RÉPONSES EN LIGNE DE LA CITÉ DE LA SANTÉ par Sandrine Lebastard et Tù-Tâm Nguyên p. 185

LE SERVICE QUESTIONS ? RÉPONSES ! DE L'ENSSIB par Pierre Moison p. 191 



\section{MODE D'EMPLOI}

par Claire Nguyen

En 1996, dans "Créer et gérer un service de référence ", dans cette même collection et sous la direction de Corinne Verry-Jolivet, Claire Stra et JeanPhilippe Lamy évoquent encore succinctement le renseignement en ligne ${ }^{1}$. À cette époque les services de référence virtuels, en plein développement aux États-Unis, n’ont pas encore abordé nos côtes. Claire Stra, par-delà le temps, semble nous passer le relais : «Ce pourrait être l'objet d'une réflexion plus approfondie, au sein des bibliothèques, dans les prochaines années ». C'est surtout à partir de 2004 que cette réflexion s'amplifie en France, accompagnant la mise en place de grands services généralistes visibles (Le Guichet du Savoir, BiblioSésame), nourrissant articles et également mémoires d'étudiants².

Des réticences ont en effet pu retarder la généralisation des services de questions-réponses en ligne ${ }^{3}$.

Il faut encore attendre 2008 pour qu'une monographie, écrite par JeanPhilippe Accart fasse la part belle aux services de référence virtuels, sans pour autant en être le sujet exclusif. Deux années plus tard, le présent ouvrage leur est entièrement consacré. Alors, pourquoi avoir fait le choix de « détacher » les services de référence virtuels des services physiques?

- Parce que ces services ont largement dépassé leur statut de "nouveau service », certains sont désormais bien rodés et leur recul et leur expérience sont indéniables. D'ailleurs, ils ne sont pas si nouveaux qu'on veut parfois le croire. Alain Jacquesson ${ }^{4}$ le redit :

1. Voir p. 22 et p. 117 de cet ouvrage. Cf. Bibliographie p. 205.

2. Dont le mien, dans le cadre de ma scolarité d'élève-conservateur à l'enssib : Mettre en place et développer un service de référence virtuel dans une perspective d'intégration à un réseau collaboratif. Cf. Bibliographie p. 205.

3. Certaines réticences ont mis du temps à se lever, notamment la peur, bien compréhensible, de se sentir envahi par tous les questionnements sauvages de la jungle du Web. L'expérience a montré que les flux étaient gérables à l'échelle de nos bibliothèques, rarement au-delà de 90 questions par mois.

4. Marie-Christine Linck, « Le renseignement personnalisé à distance », Bulletin des bibliothèques de France, t. 50, n 2, 2005, p. 99-100. [En ligne] < http://bbf.enssib.fr > (consulté le 4 avril 2010). 
« [Ce] n'est pas une pratique nouvelle. Si sa modernité tient à l'utilisation des nouvelles technologies, il n'en reste pas moins qu'il s'inscrit dans l'offre de service traditionnelle proposée aux usagers ». La référence virtuelle dans son essence est, comme la référence physique: "fonction organisée de réponses personnalisées à une demande explicite d'information documentaire ou de documentation $»^{5}$.

- Parce que les services virtuels sont autonomes, séparés ou séparables du service physique de renseignement dont ils ne sont plus seulement un appendice ou un prolongement. Il faudrait en profiter par ailleurs pour évacuer le "fantasme » qui consiste à croire que ces services à distance vont faire revenir en nombre les usagers dans les bibliothèques ${ }^{6}$. Un service de questions-réponses en ligne est donc un service ${ }^{7}$ identifié, basé sur une organisation spécifique et utilisant les nouveaux médias pour renseigner les internautes-usagers. C'est un service qui dispose de ses propres outils, stratégies, politiques, et usagers.

En outre, pourquoi ne pas avoir repris le terme, consacré par les publications successives, de "services de référence virtuels » et l'avoir remplacé par « services de questions-réponses en ligne »?

D’une part, nous avons voulu élargir la notion de " référence ", souvent restreinte au renseignement bibliographique, et l'inscrire dans la tradition anglo-saxonne, plus générale. D'autre part, nous avons voulu apparenter ces services aux autres services « questions-réponses » proposés sur le Web n'émanant pas nécessairement des bibliothèques, et qui font partie du paysage habituel des internautes. Par cette « identification d'origine contrô-

5. Bertrand Calenge. Accueillir, orienter, informer. L'organisation des services aux publics dans les bibliothèques. Cf. Bibliographie p. 205.

6. Certes, à la marge, ces services, en valorisant les ressources de la bibliothèque peuvent attirer les lecteurs, mais cela n'est ni quantifiable ni finalement si pertinent.

7. On n'abordera que peu la question du téléphone. 
lée ", les bibliothèques se posent et s'imposent dans ce monde très concurrentiel. Sans pour autant évacuer les bibliothèques ni les bibliothécaires du champ. Au contraire.

Les autres enjeux de ces nouveaux services à souligner sont justement :

- la re-intermédiation du bibliothécaire, investi du rôle d'expert validant l'information, et pas uniquement «passeur de documentation »;

- l'intégration des services de Q/R dans le fonctionnement quotidien des bibliothèques mais aussi l'intégration dans l'environnement des usagersinternautes ; les bibliothécaires vont jusqu'à s'inviter dans leur monde en utilisant le Web 2.0 (et maintenant le Web 3.0) ;

- l'incorporation du renseignement en ligne dans l'offre de service de l'établissement. Voilà l'occasion de concrétiser le nouvel esprit qui souffle dans les bibliothèques depuis quelques années : remettre l'usager au centre de nos activités ;

- l'opportunité de mutualiser les moyens et les compétences des bibliothécaires grâce aux réseaux de (questions-)réponses. Le saut collaboratif permet le bond qualitatif.

Cet ouvrage se présente donc comme :

- un manuel retraçant, décrivant, commentant toutes les étapes de la mise en place d'un service de $Q / R$ en ligne; il est construit autour de quatre parties: « Construire le projet », "Répondre: oui, mais comment ? », « Organiser son service », « Adapter son service à l'environnement et à la nature de ses usagers et de ses collections »;

- un guide des bonnes pratiques tiré d'un kaléidoscope d'expériences variées, tant françaises qu'étrangères, concernant des bibliothèques académiques ou publiques grandes ou petites, mettant en valeur des services 
spécialisés ou au contraire généralistes. Nous avons tenté de ne pas faire l'économie des cheminements, questionnements ou doutes ayant pu accompagner ces projets afin de mieux éclairer et armer la communauté des lecteurs.

La mise en place d'un service de Q/R en ligne nécessite une véritable démarche projet assise sur une réflexion préalable méthodique : analyse des besoins, état des lieux, rédaction du projet, évaluation des coûts : étapes que Jean-Philippe Accart retrace et que Caroline Bruley illustre par l'exemple de l'université de Lyon 1.

Un soin tout particulier doit être pris pour l'élaboration de la politique de service qui donne les objectifs, oriente et définit la prestation proposée. De même, il faut choisir un modèle d'organisation, adapté à la réalité de son établissement. Nous tentons de guider le lecteur dans ces deux choix. Avoir un service de Q/R, c'est bien, encore faut-il le promouvoir afin qu'il soit utilisé à la hauteur des investissements consentis. Nicolas Alarcon donne toutes les armes pour valoriser et promouvoir ces services, en s'appuyant entre autres sur l'exemple d'Ubib.fr. Grâce à Jean-Charles Houpier, nous comprenons comment l'intégration de la référence virtuelle dans l'environnement des usagers peut créer une dissémination par le biais du Web 2.0.

Pour asseoir le nouveau service et le pérenniser, il est nécessaire de l'intégrer aux autres services de la bibliothèque, notamment documentaires, ce que Bertrand Calenge démontre à partir de l'exemple du Guichet du Savoir $\left(\mathrm{GdS}^{8}\right)$ de la Bibliothèque municipale de Lyon $(\mathrm{BmL})$.

Répondre à l'usager, oui mais comment ? Marie-Françoise Defosse propose une méthodologie générale du " savoir-répondre »; celle-ci est bien particulière car il s'agit de répondre, en différé ou en temps réel, à un usager distant. Les bases de connaissances, capitalisant les questions-réponses, sont un des outils à utiliser pour mieux répondre. Marie Montano fait une 
synthèse sur ce point. Anna Svenbro donne les cadres juridiques de la réponse, que tout répondant doit avoir en tête au moment de la rédaction de sa réponse.

Il reste encore le choix de l'outil ou de l'interface de réponse. Hélène Tardif, depuis le Canada, décline toutes les solutions, de la plus simple à la plus sophistiquée, en rappelant bien que la politique et la stratégie ne doivent pas être dépendantes de l'outil. Selon les besoins et les moyens de chacun, il sera utile d'établir un tableau comparatif décliné en fonctionnalités souhaitées. Enfin, l'évaluation, développée par Nicolas Alarcon, permet d'améliorer le service et la qualité de réponse.

Comment s'organiser au mieux pour assurer la meilleure qualité de service? Sandrine Lebastard et Tù-Tâm Nguyên proposent un éventail de réunions, formations, partenariats thématiques et ciblés garants des compétences de l'équipe et du traitement de la question.

Une organisation collaborative représente un puissant moyen d'étendre et d'améliorer la qualité du service. Les réseaux collaboratifs ont en effet une carte à jouer : rendus nécessaires par la rationalisation économique, bénéficiant d'un contexte favorable (regroupements divers en PRES, communautés d'agglomérations, etc.), assis sur des infrastructures parfois existantes (consortiums d'achats, réseau de pôles associés de la Bibliothèque nationale de France $[\mathrm{BnF}] .$. ), valorisant politiquement, ils permettent l'éclosion du renseignement en ligne qui sans cela ne serait pas proposé, pas à cette échelle en tout cas ni avec cette puissance, à un échelon local. Nadine Kiker revient sur l'expérience du réseau Ubib.fr en insistant sur l'avantage et la difficulté de travailler à plusieurs voix. François Michaud expose le cas de Rue des Facs, qui s'est étendu du Quartier Latin à la région francilienne. Isabelle Copin explique le fonctionnement de Si@de, label de Q/R original.

Enfin, comment adapter son service à l'environnement, à la nature de ses usagers et de ses collections ? Quels services offrir aux usagers-internautes, selon les missions, les statuts, les collections de nos établissements ? Nous avons tenté de dresser une typologie des services de Q/R, dont les présentations de quelques réalisations «typiques » apporteront ensuite chair et 
nuance. Ainsi, pour les services généralistes, l'exemple bien connu du Guichet du Savoir (GdS) développé par Christelle Di Pietro, suivi par le SINDBAD de la BnF présenté par Isabelle Copin, et enfin le réseau BiblioSésame, projet associant la Bpi et plusieurs bibliothèques publiques, dont l'histoire et les enjeux sont narrés à deux voix, celles de Nathalie Daigne et d'Annie Prunet. Viennent ensuite en contrepoint trois services spécialisés : deux services médicaux, BIUMINFO plutôt destiné à un public d'universitaires, de chercheurs et de praticiens, analysé par Hélène GautierGentès et Questions-Santé, plus orienté grand public, que Sandrine Lebastard et Tù-Tâm Nguyên décrivent. Enfin, Pierre Moison présente le cas très spécifique du service Questions ? Réponses ! de l'enssib, destiné aux professionnels que nous sommes.

C'est donc une approche très concrète et illustrée qui est proposée ici, dans l'optique d'une Boîte à outils qui servira à toute personne intéressée par les services de $Q / R$, quelle que soit l'étape de sa réflexion : envie d'en savoir plus, projet envisagé, en préparation ou lancé, volonté d'évaluer son service ou encore de le faire évoluer. 


\section{PARTIE I}

\section{CONSTRUIRE LE PROJET}

1. LA DÉMARCHE PROJET EN VUE DE LA CRÉATION D'UN SERVICE DE QUESTIONS-RÉPONSES EN LIGNE par Jean-Philippe Accart

2. POLITIQUE DE SERVICE ET MODĖLES D'ORGANISATION par Claire Nguyen

3. LE RENSEIGNEMENT EN LIGNE AU SCD LYON 1 : LA DÉMARCHE PROJET par Caroline Bruley $++++++++++++++++++++++++++++++++++++++++++++++++$ 4. COMMENT PROMOUVOIR UN SERVICE DE RENSEIGNEMENT EN LIGNE?

par Nicolas Alarcon

5. SERVICE DE QUESTIONS-RÉPONSES ET ENVIRONNEMENT DE L'USAGER : QUELLE INTÉGRATION ? par Jean-Charles Houpier $++++++++++++++++++++++++++++++++++++++++++++++$

6. VALORISER LE SERVICE DE QUESTIONS-RÉPONSES ET L'INTÉGRER AUX AUTRES SERVICES DE LA BIBLIOTHÈQUE par Bertrand Calenge 


\section{1}

\section{LA DÉMARCHE PROJET EN VUE par Jean-Philippe Accart DE LA CRÉATION D'UN SERVICE DE QUESTIONS-RÉPONSES EN LIGNE}

\section{UNE RÉFLEXION PRÉALABLE}

Toute démarche projet induit nécessairement une réflexion préalable qui donne les bases pour comprendre les enjeux et les développements possibles dudit projet : penser et réfléchir d'emblée à un certain nombre de solutions possibles, prendre exemple sur d'autres services proposés en utilisant la méthode du benchmarking ${ }^{1}$ peuvent être très bénéfiques pour le projet. L'exemple du service de référence virtuel n'échappe pas à cette réflexion, car c'est un "nouveau » service à proposer aux usagers et utilisateurs des bibliothèques². Il doit être réfléchi, pensé, construit en fonction d'un certain nombre de paramètres. En outre, ce service se prête à toutes sortes de développements et d'enrichissements. Il utilise une approche classique de la documentation (avec les aspects " accueil », " recherche d'information ", « relation de service ») tout en étant fortement lié à la technologie (du courriel classique en passant par le chat, le forum jusqu'aux applications du Web 2.0) en vue d'établir un contact virtuel avec l'utilisateur.

\section{DÉGAGER UNE VISION ET UNE STRATÉGIE POUR LA RÉFÉRENCE VIRTUELLE}

Tout projet, quel qu'il soit, s'appuie en premier lieu sur une " vision » : il est nécessaire avant de s'engager dans la voie de la création d'un service ou d'une prestation de savoir vers quoi on tend, quel est « l'idéal souhaité ».

1. Le benchmarking est une démarche d'analyse et d'observation des meilleures pratiques employées par la concurrence et les autres secteurs sur le sujet étudié.

2. Ce service est dit « nouveau » surtout en France où il connaît un développement certain depuis quelques années. De nombreux projets sont en cours. 
Par rapport au sujet qui nous occupe, la vision du projet peut reposer sur la stratégie globale de l'institution (bibliothèque, service de documentation, archives...) en matière de service aux utilisateurs.

Prenons l'exemple de la charte Marianne de la $\mathrm{BnF}^{3}$ qui vise à simplifier et améliorer les relations entre les services publics et leurs usagers.

\section{La charte Marianne et la BnF}

Entrée officiellement en vigueur au début de l'année 2005, elle énonce cinq grands engagements qui sont déclinés en tenant compte du contexte de la $\mathrm{BnF}$ :

- « un accès plus facile aux services ;

- un accueil attentif et courtois ;

- une réponse compréhensible aux demandes dans un délai annoncé ;

- une réponse systématique aux réclamations ;

- une écoute pour progresser ».

À partir de la vision globale telle qu'énoncée ci-dessus et qui définit en même temps des objectifs généraux très utiles par la suite, il faut préciser la vision du service à développer : " La vision doit correspondre à l'image que l'on se fait du produit fini et de son concept. Pour que le projet conserve son originalité, son caractère, et qu'il atteigne ses objectifs, il est fortement conseillé qu'une personne de l'équipe soit le «porteur de la vision», afin d'assurer sa communication au reste de l'équipe, ainsi que la cohérence du produit conçu $»^{4}$. À titre d'exemple, une vision pour un service de référence virtuel peut être : « Le service de référence... est créé dans l'objectif de l'accès à l'information à distance pour tous, en fonction de la politique documentaire mise en place, dans les limites définies par le règlement ».

À l'inverse, lorsque la vision n'est pas centralisée et que chacun fait impacter sa propre vision sur le projet, on parle de design by commitee (qu'on peut traduire par « consensus mou »). Les experts s'accordent pour dire

3. La charte Marianne de la Bibliothèque nationale de France, 2010. [En ligne] < http://www.bnf.fr/fr/collections_et_services/services_lecteurs/a.accueil_orientation_public.html > (consulté le 25 février 2010).

4. In Ergologique : conseils en conception centrée utilisateur. «Porter la vision d'un projet », 2010. [En ligne] < http://www.ergologique.com/actualites/actus.php?id_actu=781 > (consulté le 25 février 2010). 
que cela a bien souvent des conséquences catastrophiques sur un projet. Afin que le projet de création de service de référence virtuel prenne corps, un certain nombre d'étapes doivent être remplies :

- la rédaction et la publication de la lettre de mission qui définit le mandat de projet ;

- la mise en place du groupe de projet avec un chef de projet ;

- l'élaboration du plan de projet et la planification du projet.

\section{LA LETTRE DE MISSION}

La lettre de mission est un document qui témoigne de l'appui de la direction, qui autorise le chef de projet à mener le projet et à attribuer les ressources nécessaires. La rédaction de la lettre de mission n'est pas compliquée. Il suffit d'indiquer le nom et le but du projet ainsi que le nom du chef de projet et une déclaration de soutien de la part de la direction. Elle est signée par la direction de l'organisme en charge et les organismes participants.

La lettre de mission doit être diffusée largement à tous ceux qui sont touchés par le projet. Cela aidera à donner un élan au projet, à renforcer l'autorité de son responsable et, possiblement, à intéresser d'autres personnes dont la collaboration pourrait s'avérer précieuse, à faire partie du groupe de projet.

\section{METTRE EN PLACE UN GROUPE DE PROJET}

La composition du groupe de projet est primordiale : c'est généralement la direction de l'institution ou un comité de pilotage des projets au sein de celle-ci qui met en place un tel groupe. Un chef de projet est désigné ${ }^{5}$ et

*. Les termes suivis d'un astérisque (à leur première occurence) sont définis dans le glossaire en fin d'ouvrage.

5. Celui-ci peut être le responsable du service physique de référence ou des services aux publics mais pas nécessairement ; on a vu des chefs de projet responsables en charge des ressources électroniques, du portail*, de la formation aux usagers... 
reçoit une lettre de mission. Peuvent être intégrés au groupe de projet un membre de la direction (qui n'est pas membre du comité de pilotage), un ou deux professionnels de référence, un membre du service de la communication, un membre du service informatique... Des personnes extérieures peuvent être consultées ponctuellement sur certains points du projet.

Une fois le groupe mis en place et le chef de projet désigné, il convient d'être très attentif à la demande de la direction de l'institution et de s'assurer de la clarté de ses engagements et de ses exigences.

Le rôle du chef de projet est essentiel : il administre les détails du projet, au jour le jour. Il s'agit là d'un défi constant qui demande une compréhension du contexte plus général du projet et la capacité de concilier des exigences contradictoires telles que :

- les ressources disponibles et les attentes (un service de référence virtuel qui vient compléter un service en présentiel peut se voir attribuer peu de moyens, hormis ceux existants. Aux professionnels de référence de trouver une solution adaptée avec un redéploiement des ressources) ;

- les priorités différentes des parties prenantes (certains peuvent voir dans le projet de service virtuel un moyen de promouvoir la bibliothèque ; d'autres une manière de remédier à une désaffection du public) ;

- les besoins définis et à la portée du projet ;

- la qualité et la quantité.

\section{CONSTITUER UN PLAN DE PROJET}

Pour qu'il soit d'une utilité maximum, un plan de projet doit être pertinent, compréhensible et tenir compte de l'importance et de la complexité du projet. Il est remis à jour régulièrement.

Il inclut les éléments suivants :

- la lettre de mission ;

- le calendrier des activités prévues ; 
- les personnes concernées et le temps de travail consacré au projet par personne (un jour par mois pendant une année : au final, cela permet de calculer régulièrement le temps global pris par le projet et ainsi de l'évaluer en termes financiers) ;

- les responsabilités attribuées (au comité de pilotage, au groupe de projet, au chef de projet) ;

- le budget prévu ;

- les étapes importantes, avec les dates cibles (contacts à prendre ; études et analyses à réaliser ; rapports intermédiaires et final à rendre) ;

- la stratégie de gestion du risque (en cas de non faisabilité du projet, de défection du chef de projet...).

Le plan de projet devra être remis à chaque partenaire et à tous les principaux membres du groupe de projet. Il s'agit d'un outil qui peut permettre d'éviter la confusion quant à la portée du projet et les malentendus sur les responsabilités, les échéanciers ou la gestion des ressources.

\section{LA PLANIFICATION DU PROJET}

Chaque projet a un cycle de vie composé d'étapes à franchir du début à la fin. Les grandes étapes de la planification d'un projet sont les suivantes :

- l'élaboration du concept, des buts et des objectifs du projet en incluant les besoins en technologie (principalement informatique), en ressources humaines et documentaires si nécessaire ;

- la réalisation de la phase initiale (évaluation des besoins, inventaire des ressources) ;

- la réflexion et la réalisation du projet pilote ;

- les tests du pilote ;

- la rédaction du rapport final et la diffusion des résultats ;

- après acceptation du projet par le comité de pilotage et/ou la direction, l'implémentation du service de référence virtuel ; 
- la mise en place de la stratégie marketing du service.

Chaque étape est concrétisée par un rapport intermédiaire rédigé par le chef de projet et approuvé par le comité de pilotage qui délivre l'autorisation de passer à l'étape suivante.

Un projet comme celui du service de questions-réponses en ligne peut faire partie d'un projet plus global, par exemple la refonte du site Web de l'institution, ce qui est le cas actuellement pour les bibliothèques de l'université de Genève qui prévoient un site Web avec un seul service de référence, point d'accès unique pour les utilisateurs ${ }^{6}$. Dans cette configuration, le projet d'origine devient un sous-projet qui doit tenir compte des échéances du projet global (par exemple, l'ouverture du service de référence virtuel coïncidant avec celle du site Web général), avec d'autres implications techniques et financières. Il faut également tenir compte des autres projets de la bibliothèque afin de déterminer au mieux le calendrier idéal (par exemple : réinformatisation, extension des horaires...).

Pour un projet touchant le public, avec la mise à disposition d'un nouveau service, il est primordial de réaliser une analyse des besoins des utilisateurs et un état des lieux de l'existant.

\section{RÉALISER L'ÉTUDE DE FAISABILITÉ ET L'ANALYSE DES BESOINS ET DE L'EXISTANT}

Un état des lieux et une analyse de la situation en matière de référence et de services en ligne proposés par la bibliothèque constituent les premières étapes du projet. Elles servent à étayer le bien-fondé d'une éventuelle création de service représentant un certain investissement, et une redistribution des ressources et du personnel. Il faut préciser d'emblée que les coûts peuvent être très variables en fonction de la solution choisie?

\footnotetext{
6. À l'heure actuelle, chacune des 8 bibliothèques de facultés dispose de son propre site Web et 4 ont créé un service de référence virtuel (droit, médecine, sciences économiques et sociales et sciences). 7. Voir le paragraphe «Les coûts de mise en place et le budget », p. 30.
} 
La proposition de créer un nouveau service pour les usagers de la bibliothèque (réguliers ou non) est la conséquence d'un certain nombre de questionnements: pourquoi créer un nouveau service? Quels sont les développements en cours dans les bibliothèques? Comment évolue le paysage informationnel ? La littérature, les journées d'étude et les congrès professionnels sont d'excellents moyens de s'informer. Cela peut être aussi le résultat du constat d'une fréquentation en baisse, du sentiment que les usagers trouvent d'autres moyens de s'informer, du souhait de compléter une offre de services déjà existante, de la volonté de travailler en réseau avec d'autres institutions et pourquoi pas au niveau de la référence virtuelle? Les raisons sont donc multiples. La demande de création peut provenir de l'équipe de référence en place ou de la direction de l'institution, ou éventuellement du souhait d'une tutelle de rendre plus visible la bibliothèque sur Internet.

\section{L'ÉTAT DES LIEUX EN MATIÈRE DE RÉFÉRENCE}

La création ou le développement d'un service de référence virtuel nécessite la prise en compte de la situation dans son ensemble et l'étude des divers aspects, à la fois des ressources humaines et documentaires, et des aspects techniques.

L'état des lieux se déroule selon plusieurs étapes, sur le plan interne et sur le plan externe.

Au plan interne :

- dresser le bilan du service de référence en présentiel quand il existe : ses forces, ses faiblesses, son utilisation ou sa non-utilisation... Le plus souvent, une bibliothèque cherche à remédier à la désaffection du service présentiel par le virtuel. Cela peut aussi représenter un développement utile et complémentaire à la fois pour l'institution et pour le personnel de référence ;

- procéder au recensement et à l'évaluation du personnel de référence en place, qui va de pair avec un recru- 
tement éventuel : niveau de formation requis, qualifications et compétences nécessaires. L'équipe de référence est-elle prête à répondre à ce nouveau défi, sachant qu'il peut y avoir des contraintes différentes d'un service présentiel?

- de plus, il faut se poser la question de l'équipe de répondants. Élargit-on le cercle des bibliothécaires faisant du renseignement présentiel (traditionnellement les catégories A et B) ? Les personnels de catégorie $\mathrm{C}$ par exemple pourraient répondre aux questions de localisation et de réorientation. Certains peuvent avoir développé des compétences en recherche documentaire. Inclut-on également des personnels de statut autre que bibliothèques (PRCE, ITRF, AENES...) qui travaillent dans les bibliothèques ; voire d'autres services non documentaires ${ }^{8}$ ? Incluton les bibliothèques associées (d'UFR pour les bibliothèques universitaires ou associatives pour les bibliothèques publiques par exemple) ? Tout dépendra de la politique et de la culture de l'établissement;

- dresser l'inventaire des ressources documentaires existantes: en termes de finances (budget), de matériels (mobiliers, fournitures), de locaux, de moyens informatiques (nombre de postes informatiques alloués, accès à des banques de données, mise en réseau des données existantes, connexion Internet), de collections de référence (ouvrages, revues, objets, documents électroniques...) : ces dernières ressources seront utiles pour répondre aux questions posées en ligne ; si elles existent en mode électronique, elles peuvent être proposées sous forme de répertoire de liens, ce qui présente l'avantage d'orienter les 
recherches autrement et de ne recueillir que des recherches plus complexes. Une Frequently Ask Questions (FAQ) ${ }^{9}$ peut aussi répondre aux questions les plus souvent posées. Cela montre qu'un service de référence en ligne peut être accompagné par différentes offres documentaires ;

- mettre en balance les divers services en ligne proposés par la bibliothèque, voir comment ils s'articulent entre eux et quelle est leur cohérence ;

- réfléchir à l'interactivité proposée par le site de la bibliothèque : existe-t-il déjà un forum en ligne, un blog* où les usagers peuvent déposer leurs commentaires?

- considérer les développements techniques nécessaires selon le type de service virtuel retenu: un chat demande une infrastructure technique différente d'un simple service d'échange de courriels ; une base de connaissances en ligne qui référence les questions et les réponses est une option plus lourde à gérer qu'une FAQ par exemple, mais apporte une réelle valeur ajoutée.

Au plan externe :

- consulter la littérature professionnelle : même s'il n'existe pas en français de titres spécialisés sur la référence ou la référence virtuelle, des articles sont publiés régulièrement dans la presse professionnelle francophone. Des titres en anglais existent ${ }^{10}$;

9. Par exemple, la FAQ d'@zimut (université de Provence) : < http://www.univ-provence.fr/gsite/ document.php?pagendx=7665\&project=scd $>$ (consulté le 3 mai 2010).

10. Voir D-Lib Magazine : < http://www.dlib.org > ;

Internet Reference Services Quarterly : <http://www.haworthpress.com > ;

Medical Reference Services Quarterly: < http://www.haworthpress.com/store/product.asp?sku=J115 > ;

Reference \& User Services Quarterly: < http://rusq.org > ;

Reference Services Review: <http://www.emeraldinsight.com/info/journals/rsr/rsr.jsp > ;

The Reference Librarian: < http://www.haworthpress.com > (sites consultés le 3 mai 2010). 
- réaliser une étude de benchmarking des services de référence existants et équivalents à celui que l'on souhaite mettre sur pied, ce qui revient à dresser un tableau comparatif avec les avantages et les inconvénients, ou les points forts et les points faibles ;

- se référer aux normes actuelles en matière de référence, normes établies par des organes officiels tels la section Reference and Information Services $\left(\right.$ RISS) ${ }^{11}$ de l'International Federation of Library Associations (IFLA) ou l'association Reference and User Services Association (RUSA) ${ }^{12}$ de l'American Library Association (ALA). RUSA publie des directives (RUSA Guidelines) ; ou l'Association of College and Research Libraries (ACRL) qui publie un guide pour les services à distance, Guidelines for Distance Learning Library Services ${ }^{13}$;

- contacter d'autres professionnels ayant déjà mis en place un tel service, les questionner pour qu'ils fassent part de leur expérience ;

- assister à des formations continues sur le sujet (notamment celle de l'enssib).

En parallèle à cet état des lieux, la collecte des besoins et des souhaits des utilisateurs à l'aide d'une enquête de besoins permet d'appréhender les différents niveaux, la diversité et la pluralité des attentes, éléments importants pour apprécier la situation et offrir des solutions.

11. RISS : < http://www.ifla.org/VII/s36/index.htm > (consulté le 3 mai 2010).

12. RUSA : < http://www.ala.org/ala/mgrps/divs/rusa/ > (consulté le 3 mai 2010).

13. ACRL : < http://www.ala.org/ala/acrl/acrlstandards/guidelinesdistancelearning.cfm > (consulté le 3 mai 2010). 


\section{L'ANALYSE DES BESOINS DES UTILISATEURS EN MATIÈRE DE RÉFÉRENCE}

Plusieurs techniques peuvent être utilisées pour l'analyse des besoins l'observation directe, l'entretien et l'enquête - soit séparément, soit en conjonction. Elles visent à effectuer une analyse des besoins d'information, mais également à mettre en avant les manques si le service existe déjà et demande à être développé. Elles permettent de comprendre de quelle manière les moyens mis à disposition sont utilisés, si ceux-ci correspondent aux attentes.

\section{L'OBSERVATION DIRECTE}

Dans le cas qui nous occupe, l'observation directe se concentre sur les usagers de la bibliothèque qui s'adressent au service de référence ou consultent en salle de lecture ou dans les rayonnages ou sont en train d'interroger un moteur de recherche en vue d'une recherche d'information. Comment opèrent-ils ? Quelle est leur méthode ? Sont-ils autonomes ou ont-ils manifestement besoin d'aide ? Quelles questions posent-ils, comment les formulent-ils ? La plupart des professionnels de référence font de l'observation directe, même sans s'en rendre compte : ils sont tout à fait capables de dresser un état des besoins de leurs utilisateurs, par constat ou déduction. L'observation directe est d'autant plus efficace si des objectifs sont fixés à l'avance. Une prise de note est utile pour se rappeler le contexte, comment les usagers réagissent ou agissent... Elle s'accompagne d'un entretien pour être plus efficace et éviter de mauvaises interprétations.

\section{L'ENTRETIEN}

Trois types d'entretien sont répertoriés :

- l'entretien non directif, celui qui est interrogé est laissé libre de s'exprimer sans limitation ;

- l'entretien directif, avec des questions directes qui laissent peu de choix à la personne interrogée ; 
- l'entretien semi-directif, intermédiaire, qui permet d'équilibrer l'entretien.

Ces deux techniques - l'observation directe et l'entretien - permettent un contact direct avec l'usager, et donc une interaction possible avec lui : le faire réagir en situation à des fonctionnalités proposées, étudier son mode de travail, considérer ensuite l'utilité d'un service de référence. Mais ils demandent du temps et de l'investissement. Le questionnaire d'enquête est pour sa part très utilisé.

\section{LE QUESTIONNAIRE D'ENQUÊTE}

Un questionnaire comprend tout ou partie des éléments suivants, dans la mesure où un questionnaire est plus ou moins anonyme :

- la fonction exercée (technique, administrative, commerciale, recherche...);

- l'âge ;

- la formation ;

- les usages documentaires : régulier ou irrégulier ; expérimenté ou inexpérimenté ; sur site ou hors site ;

- les intérêts en matière culturelle, d'éducation, de formation, de loisirs...

- les attentes en matière de services et de prestations du futur service de référence.

Pour un questionnaire entièrement anonyme, le plan général suivant peut être adopté :

1. Sources d'information utilisées (internes, externes).

2. Avis sur les services et produits documentaires actuels.

3. Besoins d'information, leur évolution.

La formulation doit être claire, les questions précises et peu nombreuses, sinon les utilisateurs ne répondent pas. 


\section{Exemple de questionnaire dans le cas d'une bibliothèque universitaire}

1. Dans quelle université êtes-vous inscrit?

2. Quelle est votre filière d'étude?

3. Quel diplôme préparez-vous?

4. En moyenne, combien de fois par semaine travaillez-vous à la bibliothèque?

5. Sinon, où travaillez-vous?

6. Utilisez-vous les ressources en ligne proposées par la bibliothèque (banque de données, revues électroniques...)?

7. Quels services de la bibliothèque sont les plus utiles pour vous ? Prêt, prêt entre bibliothèques, places de travail, cabines de travail, renseignements, recherches, formations documentaires...

8. Pouvoir interroger la bibliothèque à distance et bénéficier de l'assistance d'un bibliothécaire vous paraît-il intéressant pour votre travail ?

L'analyse des besoins des utilisateurs est différente selon qu'il s'agit d'une bibliothèque publique (l'éventail est alors très large), d'une bibliothèque universitaire (le public - étudiant, enseignant, chercheur - est plus ciblé) ou d'un service de documentation (généralement spécialisé) ou d'archives. Dans le cas de la bibliothèque publique ou d'une médiathèque qui ne peut analyser tous les besoins potentiels, la détermination de groupes cibles d'utilisateurs est une solution : par tranches d'âge, par intérêts, par professions...

Une fois testé auprès des utilisateurs et validé par les instances concernées (direction, service communication/marketing, commission de la documentation...), le questionnaire d'enquête peut être soumis aux utilisateurs par entretien (sur place), par courrier ou courriel, ou par consultation sur le site Internet de la bibliothèque. Le dépouillement du questionnaire demande $\mathrm{du}$ temps, des moyens financiers et matériels. Certaines bibliothèques et certains services de documentation sous-traitent tout ou partie de l'opération à un prestataire extérieur, et un budget doit être dégagé. Les résultats de l'enquête sont ensuite analysés et permettront d'orienter la politique de référence. C'est au professionnel que revient la décision finale de donner 
telle ou telle orientation au service de référence grâce aux indications fournies par l'enquête de besoins.

\section{LA RÉDACTION DU PROJET}

L'analyse des besoins et l'état des lieux réalisés, le chef de projet réunit et analyse l'ensemble des éléments puis propose un projet qui doit remplir les conditions suivantes :

- dresser l'historique et offrir un bilan détaillé et chiffré de la situation : personnel, budget, espaces, collection de référence, services et prestations offerts... ;

- proposer une politique de référence en accord avec les orientations de la politique documentaire ;

- fixer des objectifs selon les besoins exprimés (utilisateurs anciens et nouveaux, champs de la référence à investir, services à développer : accès en ligne, produits documentaires...) ;

- fixer des critères de choix (priorisation), examiner en détail les ressources humaines (l'aspect « compétences » est primordial) et les contraintes matérielles (budget et locaux alloués) ;

- élaborer un modèle de service de référence en adéquation avec le système d'information mis en place ;

- proposer des objectifs et des moyens en personnel (formation professionnelle ; renforcement des compétences ; nouveaux recrutements ; travail partagé en collaboration avec d'autres institutions...) et en documentation (sélection, acquisition, équipement, indexation, production, marketing) ;

- déterminer les coûts que le projet engendre ;

- fixer des échéances précises à court, à moyen et à long terme ;

- planifier les travaux à entreprendre ; 
- choisir les outils qui serviront de support au renseignement en ligne.

\section{LA PRÉSENTATION DU PROJET, LA NÉGOCIATION DES MOYENS ET LA MISE EN CEUVRE DU PROJET}

Le projet finalisé est présenté au comité de pilotage puis à la direction et aux interlocuteurs concernés, notamment l'équipe de référence. Cela sous forme écrite (par l'envoi du rapport final) et orale. À cette étape s'engage généralement celle de la négociation des moyens : il est donc important que le projet finalisé comporte plusieurs options qui peuvent être discutées, avec des implications financières différentes selon la solution technique choisie (acquisition ou non d'un logiciel de référence par exemple...). Une fois accepté, le projet du service de Q/R en ligne sera présenté en interne, dans l'institution afin d'en montrer les avantages et de rallier les éventuels indécis : un nouveau service doit être porté par l'ensemble de l'institution et de ses membres qui, ensuite, le feront vivre et se développer.

Il convient ensuite de présenter la mise en œuvre du projet selon le modèle définitivement retenu et de procéder à une évaluation des coûts définitifs. Le projet peut alors entrer en phase de réalisation avec des échéances fixées (à court, moyen et long termes) en fonction des moyens attribués et des développements prévus.

\section{LES COÛTS DE MISE EN PLACE ET LE BUDGET}

Dans la majorité des cas, la création d'un service à l'usager bénéficie d'un budget pris sur celui d'un service ou d'un département et plus globalement sur celui de l'institution. Avec le service de référence virtuel, le cas peut se présenter différemment s'il s'agit de participer à un réseau existant ou d'en être à l'origine. Le service Rue des Facs par exemple a bénéficié d'une subvention de la Ville de Paris. Une agglomération, un département, une région peuvent tout à fait être sollicités pour financer le projet. Aux coûts de mise en place avec l'équipement du service, il faudra évaluer le budget de fonctionnement annuel nécessaire incluant les éléments ci-dessous. 
Un service de référence, qu'il soit en présentiel ou virtuel, a des charges identiques à celles d'un service administratif : le personnel, l'espace, les fournitures, l'entretien des matériels en font partie. Il faut ajouter à cela les budgets nécessaires au maintien de la collection de référence comprenant des outils papier et numériques à mettre à jour régulièrement afin de répondre aux questions de manière efficace.

\section{LES COÛTS EN PERSONNEL}

Ils sont constitués par la masse salariale et les charges non salariales :

- le salaire, incluant les charges salariales, égales à $20 \%$ du salaire brut, le nombre d'heures annuelles de travail (congés compris), et les charges patronales, soit $50 \%$ du salaire pour un plein-temps ;

- les charges non salariales: inhérentes au fonctionnement de l'entreprise (comité d'entreprise, comité d'hygiène, de sécurité et des conditions de travail [CHSCT]...).

Selon le type d'organisation choisie, les implications financières ne sont pas identiques : l'étude de faisabilité doit permettre d'évaluer les coûts à mesurer, notamment en termes de personnel supplémentaire, ou de division du travail au sein de l'équipe de référence. Elle doit comporter un volet financier qui détaille les postes de travail nécessaires, ainsi que les pourcentages de travail pour que le service virtuel puisse fonctionner efficacement. Un réseau de référence virtuel, quant à lui, permet de répartir les coûts en personnel et techniques sur un certain nombre de partenaires. Le temps de travail est à calculer avec soin pour chaque membre de l'équipe de référence, en fonction des heures d'ouverture du service de référence virtuel. Le temps passé à répondre aux questions est également à mesurer, afin de ne pas excéder les possibilités du service : le fait de déterminer à quoi le service répond et à quoi il ne répond pas présente une incidence financière non négligeable. Le coût moyen d'une réponse peut être calculé en divisant le nombre de questions par le coût total généré par les temps de travail consacrés aux réponses. Aux États-Unis, le coût d'une question 
(incluant les éléments cités précédemment) est évalué à 80 euros (108 dollars) ${ }^{14}$. D'autres tâches sont à évaluer :

- l'actualisation régulière des pages Web ;

- la mise à jour des FAQ ;

- la mise à jour des signets ; la veille ;

- le contrôle régulier de la base de connaissances...

\section{LES COÛTS LIÉS À L'ESPACE}

Il s'agit du loyer, de l'électricité, du chauffage, de l'entretien, de la participation aux surfaces communes non affectées. Ces coûts se traduisent par une évaluation au mètre carré et une répartition au millième.

\section{LES COÛTS LIÉS AUX FOURNITURES, À L'ENTRETIEN DU MATÉRIEL ET AUX PRESTATIONS DIVERSES}

Ce sont : les fournitures de bureau, la location éventuelle et la maintenance de photocopieurs, les frais de courrier et de téléphone, la maintenance informatique, la maintenance de certains appareils particuliers (lecteurs de microfiches...), les frais d'amortissement issus des investissements précédents, les frais de sous-traitance.

\section{LE COÛT DES ACQUISITIONS POUR LA COLLECTION DE RÉFÉRENCE}

Il peut s'agir : des achats ponctuels d'ouvrages, de numéros spéciaux de revues, de supports particuliers (cédéroms, DVD, microfiches...) ou des achats dits permanents, comme les abonnements aux périodiques ou les interrogations de banques de données.

Les frais de personnel sont généralement estimés à $60 \%$ du total, l'espace de 25 à $30 \%$ et les fournitures à $10 \%$.

Selon la solution technique choisie, le budget et l'investissement financier seront différents.

14. «Quel est le coût d'une question de référence ? » Voir la réponse de William H. Wisner dans Library Journal du $1^{\text {er }}$ octobre 2008 : < http://www.libraryjournal.com/article/CA6598070.html > (consulté le 3 mai 2010). 
- L'emploi d'un logiciel de messagerie simple pour les transactions de référence n'induit pas de coûts supplémentaires.

- L'acquisition d'un logiciel commercial ou d'une plateforme Web présente un coût très différent selon les fonctionnalités offertes : le logiciel QuestionPoint - qui en offre de nombreuses - a un coût de 730 euros par année (chiffre 2008). Une étude comparative, des tests, une mise en concurrence doivent être entrepris. À ce coût d'achat doivent être ajoutés les coûts de formation du personnel, ceux de la maintenance et des mises à jour tant de l'équipement que du logiciel luimême. Un serveur dédié, selon l'importance du service en ligne, est à prévoir ${ }^{15}$.

- Le développement d'un logiciel open source implique la collaboration du service informatique et représente également un coût à évaluer.

\section{EN CONCLUSION}

La démarche de projet du service de référence virtuel est comparable à celle d'un autre projet, elle répond à des règles précises, suit des étapes prédéfinies et s’inscrit dans un processus qui en garantit la réussite. Même si elle prend du temps et qu'elle mobilise un nombre de personnes parfois important, elle est nécessaire pour envisager tous les aspects du projet, son développement tout en considérant le meilleur service possible à rendre à l'utilisateur.

15. Claire Nguyen estime que les coûts peuvent varier « de 0 à 80000 euros » in Mettre en place et développer un service de référence virtuel dans une perspective d'intégration à un réseau collaboratif, ibid., p. 22. Cf. Bibliographie p. 205. 


\section{POLITIQUE DE SERVICE ET \\ par Claire Nguyen MODĖLES D'ORGANISATION}

L'un des préalables de tout projet de service de questions-réponses en ligne est la définition d'une politique de service, qui influera de manière plus ou moins directe sur son organisation.

\section{LA POLITIQUE DE SERVICE}

La politique du service définit les missions et les objectifs, le périmètre du service (en termes de publics et de thématiques), le niveau des réponses, les engagements des bibliothécaires et le cas échéant du lecteur.

Elle détermine la prestation qu'un utilisateur peut attendre. Elle peut se présenter sous forme de charte suivant là l'exemple des chartes documentaires ; elle peut en outre reprendre tout ou partie des éléments de la charte interne du service (qui définit les règles et l'orientation des réponses et des répondants). Point essentiel qui permet de lever les éventuelles ambiguïtés entre bibliothécaires et usagers, ou même entre bibliothécaires : la politique doit être élaborée et discutée bien en amont de l'ouverture du service, avec la direction de la bibliothèque, voire de l'institution, et de l'équipe des répondants.

\section{ENCADRÉ 1 LA POLITIQUE DE SERVICE EN 9 POINTS}

Quelles missions?

Elles peuvent être très générales, par exemple : "le service aide/assiste les usagers dans leur recherche et leur évaluation d'information ». On peut les formuler en termes d'objectifs, sous forme de dialogue avec le lecteur «À quoi ça sert »? «Qu'est-ce que le service X... ?» «Pourquoi ?».
À qui répond-on?

Il faut réfléchir au public que l'on vise : membres d'une communauté universitaire, lecteurs inscrits en bibliothèque publique, voire citoyens d'une ville, d'une région, d'un pays, d'un bassin linguistique. $\mathrm{Ou}$, le plus largement possible, tout internaute désireux de trouver une information validée et pertinente... 
Ce qui nous amène à poser une question, un peu différente : est-ce un public exclusif qui pourra poser des questions ? Poser ce principe revient à restreindre le service a priori. À l'heure du Web, il peut sembler étonnant de limiter ce type de service en ligne. Certaines bibliothèques restreignent l'accès de leur service et demandent une authentification. Il est en effet dommage qu'un établissement avec une mission de service public soit fermé de cette manière ; cela peut être même discriminant $^{1}$. Cette restriction handicape des lecteurs extérieurs qui demanderaient des informations sur des collections non localisées ailleurs. Selon moi, cela donne l'image d'un service-forteresse, ce qui va à l'encontre de la philosophie de ce type de service. On voit rarement le cas inverse, comme par exemple le service Questions? Réponses! de l'enssib qui exclut ses élèves... supposés solliciter les ressources de l'école sur place.

Afficher des priorités en termes de public cible peut tout à fait aller de pair avec l'ouverture à tous du service. Si besoin, on réorientera les usagers extérieurs, ou on modulera par exemple le niveau de la réponse, qui serait moins approfondie. Certains services réservent certains modes de questions à leurs affiliés : à l'université de Cornell $^{2}$, le chat est réservé à la communauté universitaire, le courriel est par contre à la disposition de tous. L'école polytechnique de Lausanne répondra différemment selon et le profil et le statut de l'usager: on ne répond pas à un étudiant non affilié comme à un chercheur de l'école, ou à un étudiant affilié et à un chercheur affiliés. Toute autre discrimination est à considérer avec une extrême précaution $^{4}$.

Il faut donc bien clarifier la politique de réorientation ${ }^{5}$ des questions reçues ou des utilisateurs. La manière dont on régule les flux le cas échéant doit être indiquée (ainsi Le GdS ferme son service à partir d'un certain seuil de questions en cours de traitement).

\section{Quand l'usager peut-il poser sa question, quand peut-il avoir une réponse ?}

Cela dépendra du mode choisi, synchrone ou asynchrone (cf. le tableau 1 p. 148). En mode asynchrone, l'usager peut poser sa question à tout moment sauf en cas de fermeture du service (congés de Noël ou d'été) pendant laquelle il ne pourra pas interroger les bibliothécaires. En mode synchrone, l'usager pourra poser sa question selon des jours et une plage horaire définis, plus ou moins étendus (ex. pour

1. Cf. l'article de C. Di Pietro, « Construire un service de référence généraliste : l'exemple du Guichet du Savoir de la Bibliothèque municipale de Lyon », p. 152.

2. Cornell (E.-U.) : < http://www.library.cornell.edu/ask > (consulté le 27 mars 2010).

3. L'école polytechnique : < http://library.epfl.ch/docs/pdf/vid_charter.fre.pdf > (consulté le 29 mars 2010).

4. Et poserait des questions déontologiques.

5. Cf. l'article de S. Lebastard et T.-T. Nguyên, « Comment s'organiser en interne ; le savoir-être du répondeur », p. 120. 
Ubib.fr : $9 \mathrm{~h}-18 \mathrm{~h}$ du lundi au vendredi). Il est plus intéressant en termes de qualité, de visibilité et de pertinence du service d'avoir une plage horaire suffisamment longue et bien placée dans la journée, sachant que le chat est plus généralement utilisé l'après-midi ou en début de soirée. Si le chat ne dispose pas d'une très grande plage d'ouverture, il est souhaitable de bien informer des autres modes de communication et de leurs heures d'ouverture, sur une même page Web si possible $^{6}$.

$\mathrm{Au}$ niveau du délai de réponse - qui concerne la réponse en différé - il faut être très clair : donner un délai maximum en nombre de jours ouvrés. En général, il s'agit de 2 ou 3 jours (48 ou 72 heures). Les utilisateurs peuvent trouver ce délai de 72 heures un peu long, mais dans le cadre d'un réseau de réponses où réorientation et réattribution des questions sont pratiquées, ce délai peut parfois être compliqué à tenir. Même difficulté si la question est complexe, et/ou posée à une bibliothèque spécialisée. Si la réponse excède le délai annoncé, il faut en avertir l'usager. Certains services conseillent même de reposer la question si elle n'a pas été traitée dans les délais impartis ${ }^{7}$ ! Pour le chat, si le bibliothécaire ne peut prendre en charge l'utilisateur, il faut envoyer un message d'attente.

\section{À quoi répond-on... et pas ?}

Ce choix dépend de chaque service. On peut définir soit les questions auxquelles on répond soit définir les questions auxquelles on ne répond pas, soit les deux. En général, sont exclues pour des raisons légales les questions s'apparentant à des consultations médicales ou juridiques ; sont exclues pour des raisons de périmètre les questions relatives à des jeux concours, aux devoirs scolaires (mais pas toujours) ${ }^{8}$, aux demandes de généalogies. Des sites de réorientation sont souhaitables (associations de patients pour une BU médicale...). On peut aussi réorienter des questions qui pourraient s'adresser à d'autres services internes de l'établissement (scolarité, informatique) et de la bibliothèque (suggestions d'achat, $\mathrm{PEB}$, circulation des documents...), sauf si on choisit de faire du service de Q/R le point de contact principal de la bibliothèque.

On peut aussi exclure des questions ne relevant pas des spécialités ou thématiques de la bibliothèque ou tout simplement de sa politique. Dans un large sens d'accessibilité, la BmL a décidé par exemple de n'exclure aucune question arrivant sur Le GdS (sauf pour des raisons légales) questions pratiques comprises.

On peut aussi choisir sa langue de réponse (couverture linguistique) : on répondra en anglais, allemand, etc. - cela dépendra

6. Par exemple, à l'université de Concordia : < http://library.concordia.ca/help/questions/ > (consulté le 27 mars 2010).

7. AskScotland (GBR) : < http://scone.strath.ac.uk/ask/hfaq.cfm > (consulté le 20 mars 2010).

8. Anyquestion (Nouvelle-Zélande) : < http://www.anyquestions.co.nz/?page_id=14 > (consulté le 3 mai 2010). 
des compétences du personnel. Le niveau de la réponse dépend également de la politique de service (cf. infra) ${ }^{9}$.

\section{Que répond-on, comment répond-on?}

Avant tout, il faut décider si on donne une réponse brute à la question et/ou si on indique la méthodologie à suivre. Ce choix dépend de chaque bibliothèque.

Quelle que soit la profondeur de la réponse, les bibliothécaires, prudence oblige, se défendent de prétendre à l'exhaustivité et à la vérité. En outre, la profondeur, la complétude, est à moduler selon le profil de chacun ${ }^{10}$. On peut l'exprimer en termes chiffrés : SINDBAD et Rue des Facs indiquent des limites en termes de temps de recherche et de contenu ( 1 à 2 heures et 15 références pour le premier; 10 références pour le second).

\section{La protection des données}

Il convient d'afficher clairement la politique de protection des données (privacy policy) et leur anonymisation ${ }^{11}$.

\section{Engagements du lecteur}

Certains services demandent au lecteur de signer "virtuellement » une charte où il garantit son bon comportement et/ou accepte les conditions d'utilisation, comme on le voit sur certains forums. Comme tout service sur le Web, les services de Q/R sont exposés à tous. Le service, en droit d'exiger un comportement acceptable, peut afficher les conditions d'exclusion du lecteur (insultes, diffamation...). Dans les faits, de tels agissements restent rares.

\section{Une politique de service volontaire}

Distiller d'autres informations comme indiquer qui répond (avec ou sans photos) - sans donner nécessairement d’informations nominatives - valorise l'équipe de bibliothécaires. Dans la même logique, donner de brefs éléments du fonctionnement du service à titre informatif.

Une rubrique d'aide au service («Comment poser ma question »), des informations sur l'équipement nécessaire le cas échéant (logiciel, navigateur, plug$i n^{*}$ ) sont souhaitables. Cette rubrique peut se décliner sous forme de FAQ. Autre type de FAQ, celle qui sous forme de questions typiques ou des questions les plus posées donne à l'usager une idée de l'éventail des questions-réponses possibles.

\section{La politique des réseaux de (questions-)réponses}

Dans le cadre de réseaux collaboratifs, le souci majeur est d'harmoniser les politiques des uns et des autres, sans tomber dans une politique minimaliste en cherchant des dénominateurs communs ${ }^{12}$. La

9. Cf. l'article de M.-F. Defosse, « Comment répondre et qu'est-ce que bien répondre ? Construire le savoirrépondre », p. 78.

10. Cf. l'article de M.-F. Defosse, ibid.

11. Voir l'article d'A. Svenbro, « Le cadre juridique des questions et des réponses », p. 92.

12. Cf. l'article de N. Kiker, « Travailler ensemble : l'exemple d'Ubib.fr », p. 130. 
charte interne est particulièrement à soigner pour rendre le service homogène. Les politiques des réseaux, par rapport aux politiques des services locaux, mettent plutôt l'accent sur les missions de ce réseau et donnent des informations sur les participants. Ils disposent en général de sites Web dédiés. AskScotland a ainsi imaginé une carte interactive des bibliothèques participantes, beaucoup plus ergonomique qu'un fastidieux annuaire ${ }^{13}$.

\section{VISIBILITÉ DE LA POLITIQUE}

La Charte doit être rédigée en termes très clairs, compréhensibles par l'utilisateur, en évitant tout jargon ; on peut la formuler sous forme de questions-réponses dynamiques. Même si des limites - ne décourageant pas le lecteur ! - sont posées, la politique ne doit pas être conçue en termes négatifs.

On peut la fractionner, la répartir sous divers onglets ou rubriques, séparer la politique de confidentialité. La politique peut s'appeler « en savoir plus », "FAQ » (terme très polysémique !), " conditions d'utilisation », " service » tout simplement, « charte » (dont l'appellation appelle un engagement de la bibliothèque) ou sans nom spécifique (reprise du titre du service en haut de page). Plusieurs choix d'accès à cette politique sont possibles : mettre sur la même page Web le formulaire de réponse, faire une page intermédiaire obligatoire avant d'accéder à ce dernier, ou à l'inverse valoriser le formulaire de réponse en « rejetant » la politique dans le menu, donc en « lecture optionnelle $»^{14}$.

Pour gagner en clarté sans perdre en quantité d'informations, on pourra exposer en quelques points percutants la politique de service, et proposer un lien vers une charte plus longue (où pourra s'insérer tout ou partie des éléments de la charte interne utiles aux professionnels).

\section{... ET APPLICATION !}

Une charte engage à une prestation minimum qui ne signifie pas minimale. Libre aux bibliothécaires de l'assouplir s'ils le jugent nécessaire ; en

13. AskScotland : < http://www.scotlandsinformation.com/SIShow.cfm?TI=18\&ST=1\&MC=sca,typ,ove,pzl > (consulté le 3 mai 2010).

14. Voir l'article de N. Alarcon, « Comment promouvoir un service de renseignement en ligne? ? p. 52. 
revanche, la durcir serait moins heureux. Il restera toujours des situations délicates, comme le cas de classes entières qui viennent poser des questions au service, cas dont il faudrait prévoir l'éventualité dans la charte interne (quels seront les niveaux de réponse et la réorientation apportés ?).

\section{ORGANISATION DU SERVICE}

Comme pour la politique de service, il faut décider suffisamment tôt de l'organisation. Cette dernière dépendra, entre autres, des modes choisis (synchrone et/ou asynchrone), de la taille de l'équipe et de sa structuration (équipe spécialisée de référence dédiée, préexistante ou non), du type d'établissement, de l'équipement et de l'espace mis à disposition et du nombre de questions susceptibles d'être posées.

Il faut prévoir un modèle pilote à réajuster, ne pas hésiter à amender, corriger, changer l'organisation.

\section{UN SERVICE PARTICULIER ?}

Intègre-t-on le service de $Q / R$ au service " physique » de renseignement sous l'égide du même responsable ? Cela impliquerait que tous les bibliothécaires qui répondent en présentiel répondraient systématiquement à distance. $\mathrm{Ou}$ en fait-on un service autonome avec un recrutement propre (sur la base du volontariat ou pas) ? Ces choix relèvent de l'établissement. Il faut avoir à l'esprit plusieurs considérations :

- le volontariat est séduisant, notamment au moment du lancement du nouveau service qui bénéficiera de l'enthousiasme de l'équipe. Cependant, c'est un élément de fragilisation pour sa pérennité qui variera au gré des mutations ou des changements d'intérêt. La stabilité d'un service doit passer par l'inscription dans la fiche de poste. De plus, si la charge de travail devient trop importante, cela peut entraîner des tensions entre répondants et non-répondants ;

- l'inscription dans la fiche de poste est importante : elle permet de quantifier et de formaliser la participation du répondant. Elle ne sera que plus efficace si elle 
prend en compte un allégement des autres tâches. Trop souvent malheureusement, en cas de surcharge de travail, le renseignement en ligne passe à la trappe ;

- le nombre de répondants est à bien évaluer. Si les répondants sont trop nombreux, ils répondront peu souvent, et risqueront de " perdre la main » et de se démotiver ; pas assez de répondants, et c'est la panique, le surmenage et, résultat identique, la démotivation. Répondre à au moins une question par semaine permet de maintenir son savoir-faire ;

- il est préférable de ne pas handicaper d'emblée les répondants : mettre en place, à l'image du service physique, un système de suppléance et de remplacements, de renforts lors des absences (congés, maladies) ;

- il faut choisir si on assigne les réponses selon les spécialisations des bibliothécaires, leur activité et leurs compétences et connaissances personnelles.

\section{MODES D'ORGANISATION EN FONCTION DU LIEU DE RÉPONSE ET DU MOMENT}

\section{UNITÉ DE LIEU}

\section{Mode intégré : à partir du bureau de renseignement}

Le service de Q/R est intégré au bureau physique de renseignement (BR) : les bibliothécaires répondent de cet endroit, en mode asynchrone ou synchrone : les permanences coïncident. Cela permet de meubler les temps morts - à condition que les pics d'activité du renseignement virtuel et physique soient différents -, d'être proche des sources imprimées de référence. Cependant, ce modèle est potentiellement générateur de stress si le bibliothécaire doit jongler entre deux usagers - minimum - (« physique » et « virtuel ») ; alors, à qui donner la priorité ? Le lecteur pourrait voir d'un mauvais œil le bibliothécaire chatter en ligne et se sentir délaissé (quoique, la promotion de ce service serait aussi faite !). D'autre part, on ne finit pas forcément sa réponse dans les limites de sa permanence, il faut rester 
au BR après sa plage horaire, ou terminer dans son bureau (pas commode en mode synchrone). D'ailleurs, le mode de réponse en différé se prête plutôt bien au mode intégré et dans des bibliothèques dont les BR ne sont pas très fréquentés.

\section{Mode centralisé : à partir d'un bureau virtuel séparé}

Le bureau virtuel dédié au renseignement en ligne est séparé, le bibliothécaire se consacre aux usagers-internautes. Les répondants, en alternance, peuvent travailler d'un local spécifique (d'où ils répondent aussi au téléphone). Ils sont plus au calme, avec des documents de référence également à portée de main. Cependant, il faut du personnel et de l'espace ; il peut immobiliser des agents si aucun usager virtuel ne se présente. Il est donc plutôt adapté aux grandes structures qui disposent de personnel conséquent et dont le service de $Q / R$ est déjà bien sollicité, notamment lorsque les périodes de pointe du renseignement présentiel et distantiel coïncident.

\section{Mode affranchi : à partir d'un lieu non affecté}

Il n'y a pas de lieu spécifique affecté aux répondants. Ce modèle est plus souple : le répondant effectue ses tâches depuis son bureau. Il peut donc s'affairer à ses autres activités quand il ne répond pas. Il doit donc être attentif aux nouvelles questions (signal sonore, visuel...). Ce modèle est économique en personnel, en espace et en équipement. Cependant, le travail se fait de manière impalpable, " cachée »; des frustrations sont susceptibles de naître du manque de visibilité, et a fortiori de reconnaissance, de cette tâche.

Des variantes de ces modèles existent bien entendu. Ainsi, à l'université de Duke (États-Unis), les bibliothécaires répondent de leur bureau, puis au BR à partir de 18 heures.

Un ordinateur portable connecté au Wifi pourrait être dédié au service, permettant aux répondants de se déplacer dans la bibliothèque si la question de l'usager le requiert.

\section{sine loco : hors les murs...}

Le répondant peut répondre à distance avec certaines interfaces. Avec l'augmentation des ressources électroniques accessibles à distance, on peut être 
tenté - ou non - de répondre de chez soi... ${ }^{15}$ Certains établissements pour assurer des plages de chat très étendues rémunèrent des bibliothécaires en heures supplémentaires pendant la fermeture de la bibliothèque, d'autres ont recours à des répondants " mercenaires » (les after dark operators d'Ohiolink).

\section{UNITÉ DE TEMPS : RÉPARTITION DES QUESTIONS ET PLANNINGS Le mode dédié}

Le temps de la réponse est dédié ; on répond selon un système de planning ; les plages horaires peuvent coïncider avec celles du bureau de renseignement (cf. mode intégré) ou non. La longueur des plages peut varier selon :

- le mode. Exemple : un jour pour le différé, 2 heures pour le chat, 1 semaine pour Facebook... ;

- la fréquentation: plages plus courtes ou doublées en cas d'affluence ;

- renfort le lundi pour répondre aux questions arrivées pendant le week-end.

\section{Le « mode partagé » ou au fil de l'eau}

On répond au fur et à mesure de l'arrivée des questions, selon ses disponibilités. C'est le cas pour BIUMINFO - même si chacun est tenu de consulter avec régularité l'interface de gestion. Le coordinateur assigne les questions laissées en suspens. Ce mode très souple s'appuie surtout sur la bonne volonté et n'est applicable que si le nombre de questions reste gérable.

\section{Le mode distribué}

Les questions sont centralisées par un coordinateur qui les réaffecte selon les spécialités ou les disponibilités de chacun (SCD de Reims, de l'enssib et du réseau BiblioSésame).

15. Je me souviens d'un stagiaire participant à la même formation que moi, en 2009, qui a dû s'éclipser pour assurer sa plage de chat, à des centaines de kilomètres de son établissement. 


\section{Le mode réparti}

Les questions sont attribuées a priori, sans l'intervention d'un coordinateur, à partir par exemple de la sélection de l'utilisateur dans le menu déroulant thématique ou disciplinaire (s'il choisit par exemple " Histoire », sa question sera envoyée aux personnes en charge des questions de la discipline). C'est le cas de SINDBAD qui s'appuie sur la départementalisation de la BnF ou de Rue des Facs.

Là aussi, les variantes sont nombreuses. Le GdS repose sur un modèle hybride, en même temps centralisé et décentralisé, puisque les questions, qui ne sont pas traitées par le service dédié (3 personnes), sont redirigées vers les départements dont c'est le domaine d'expertise. Ces modes ne sont pas non plus exclusifs : Rue des Facs fonctionne en mode réparti (questions envoyées au pétale thématique et partagé au sein du pétale).

Ces différents modes sont aussi adaptables aux réseaux collaboratifs de réponse.

\section{LES RÉSEAUX COLLABORATIFS ${ }^{16}$}

Au sein d'un réseau chaque établissement est, en général, libre de son organisation interne ; la responsabilité du service se fait au niveau de l'établissement.

Les contributions sont proportionnelles à la taille de l'établissement ou à ses possibilités ${ }^{17}$.

\section{Quelle coordination ?}

Toute la richesse et la difficulté des réseaux résident dans le travail à plusieurs. En général, se constituent des comités à deux niveaux de responsabilité : le comité de pilotage qui définit la politique, fixe objectifs et orientations (directeurs des bibliothèques ou leurs représentants), un ou des groupes de travail, plus techniques qui s'occupent par exemple du fonctionnement, des formations, des interfaces, de la gestion... Il y a 1 groupe de travail pour Ubib.fr, 7 groupes thématiques AskUsNow du Maryland ${ }^{18}$. La

\footnotetext{
16. Voir l'article de C. Nguyen, « Typologie des services de questions-réponses », p. 146.

17. Par exemple, la Bibliothèque nationale australienne assure 20 heures de permanence par semaine, les petites bibliothèques 1 heure, dans le cadre du réseau AskNow.

18. AskUsNow : < http://www.askusnow.info > (consulté le 28 mars 2010).
} 
coordination générale peut être assurée par un ou plusieurs établissements (comme pour la Deustche Internet Bibliothek en Allemagne, entre la bibliothèque publique de Dresde et de l'État du Bade-Wurtemberg). Les bibliothèques coordinatrices peuvent bénéficier de décharges horaires (moins de permanences de réponse) ou de réductions tarifaires sur l'acquisition du logiciel collaboratif. Certains réseaux se dotent de têtes de réseau comme c'est le cas de certaines bibliothèques nationales (Australie, etc.) ${ }^{19}$. Ce terme peut gêner, entre établissements de taille comparable, ou pour des raisons politiques ; on préférera alors le terme coordination, voire de secrétariat, assumés à des degrés divers.

\section{Penser à l'articulation entre local et global}

Les membres d'un réseau ont parfois des services locaux pré-existants qu'ils peuvent vouloir garder, totalement ou partiellement ; par exemple, le chat peut être local et le différé collaboratif (Bpi) ou inversement. Rien n'interdit non plus la participation à plusieurs réseaux.

L'articulation entre local et global doit aussi être pensée en termes d'investissement : des bibliothèques qui répondraient en majorité à des usagers non locaux pourraient être mécontentes de ce déséquilibre.

C'est cette crainte et la volonté forte d'ancrer localement les services de la bibliothèque qui peuvent expliquer le maintien de services locaux et déterminer des degrés d’implication variables. Je vais présenter les modèles collaboratifs par ordre « croissant » d'implication (qui ne rime pas avec motivation!). 


\section{ENCADRÉ 2 LES MODĖLES COLLABORATIFS}

\section{La logique d'appoint}

La bibliothèque répond à sa « file » locale et partage les questions avec les autres membres en dehors des heures d'ouverture (bibliothèque américaine de Portland - Oregon Libraries Network). Elle est parallèle à la logique de secours : chaque bibliothèque gère sa file et partage le surnombre avec les autres bibliothèques en cas de saturation.

\section{La logique coopérative}

La bibliothèque traite les questions qui lui sont adressées et renvoie les questions auxquelles elle n'est pas parvenue à répondre ou pour lesquelles elle n'est pas spécialisée (Si@de).

Le logiciel QuestionPoint prévoit pour ses abonnés la réorientation automatique des questions sur le réseau mondial à la bibliothèque la plus à même de répondre.

\section{La logique répartitive}

Les questions sont envoyées a priori aux bibliothèques/bibliothécaires selon leur nature et le profil de la bibliothèque/du bibliothécaire membres (ex: pétales thématiques de Rue des Facs ou d'Ubib.fr en mode différé).
La logique est distribuée quand le coordinateur général répartit les questions à leur réception (Bibliosésame).

\section{La logique mutualiste}

Les bibliothèques répondent aux questions à tour de rôle selon des plages variables (en 2006: une fois par semaine pour Pregunte, Esp). Le réseau médical britannico-australien Chasing the Sun se répartit les plages selon les fuseaux horaires. Cette organisation est plutôt utilisée pour les services en temps réel.

On voit là différents modes, modèles, modalités de l'organisation de ces services. À chacun de puiser selon son environnement, ses objectifs, sa culture d'établissement et sa propre réalité. 


\section{3}

\section{LE RENSEIGNEMENT EN par Caroline Bruley LIGNE AU SCD LYON 1: LA DÉMARCHE PROJET}

Service de renseignement en ligne (REL)

$<$ http://mvdoc.univ-lyonl.fr/Portail/Site/Typo3.asp?lang=FR\&id=ucbl-reldemande >

\section{ANALYSE DES BESOINS ET DE L'EXISTANT}

Le service de renseignement en ligne (REL) était l'un des nouveaux services prévus dans le cadre de la mise en place du portail documentaire de Lyon 1. Il a répondu à un double besoin.

Celui de renseigner le lecteur : avec l'utilisation massive d'Internet et des ressources électroniques en bibliothèque, il nous semblait logique et nécessaire de prolonger le renseignement au-delà des murs de la bibliothèque et d'être également présent en ligne. Un besoin plus ponctuel, découlant de la fermeture de la bibliothèque des sciences durant les travaux de réhabilitation du bâtiment (fermeture en juin 2007, ouverture d'une BU provisoire en octobre 2007, réouverture de la BU Sciences en septembre 2009). Durant ces 4 premiers mois sans BU, puis durant les mois de travaux où les conditions d'accueil ont été difficiles, il fallait que les étudiants de sciences puissent facilement poser leurs questions tant pratiques que documentaires, et un REL semblait la solution idéale.

\section{QUEL CALENDRIER ?}

L'ouverture du REL devait coïncider avec l'ouverture du portail documentaire de Lyon 1 et la fermeture de la BU Sciences (juin 2007). Mais le portail a connu de gros retards, il a donc fallu monter un premier REL sur le site Web en fin de vie de la BU et le transposer ensuite dans le nouvel environnement du portail documentaire de Lyon 1 . 
$\mathrm{Au}$ final, le calendrier a été le suivant :

- janvier/février 2007: début de la réflexion autour d'un REL par le groupe projet portail documentaire ;

- mars 2007 : constitution d'un groupe de travail REL, aussi diversifié que possible en termes de section d'appartenance, de statut et de compétences ;

- mars/mai 2007 : travail autour de l'organisation du service, des interfaces publique et professionnelle ;

- juin 2007 : création du formulaire et de la page d'accueil du service sur le site Web du SCD ;

- 18 juin 2007 : ouverture du service au public et campagne de communication via des flyers, des actualités sur les portails étudiants et documentaires, des dépliants ;

- octobre/décembre 2007: étude de la transition du REL $\mathrm{du}$ site Web vers le portail documentaire par les groupes projet portail et REL ;

- début février 2008 : ouverture du portail documentaire et du REL associé.

\section{QUELS LOGICIELS ET QUELS COÛTS ?}

Pour le REL site Web (juin 2007/janvier 2008), aucun logiciel n'a été nécessaire, nous avons simplement créé un formulaire en ligne sur une page KSup du site Web (2 jours de travail pour la $\mathrm{BAS}^{1}$ membre du groupe projet portail).

Pour le REL portail documentaire (à partir de février 2008), nous avons utilisé l'outil à workflow* simple fourni par la société INEO Media System, détentrice du marché. Il est impossible de chiffrer le coût de l'outil, de la maintenance associée et de la formation au logiciel, ces frais étant totalement imbriqués dans le coût du projet. Les seuls coûts liés ont été les coûts humains : travail du groupe projet et participation des bibliothécaires au REL. 


\section{QUELLE POLITIQUE DE SERVICE ?}

Le public cible : il a rapidement été décidé de laisser ce REL ouvert à tous, et pas seulement aux usagers de Lyon 1, pour deux raisons. Premièrement, nous n'étions pas une BM, le nombre de questions s'annonçait donc limité, même en mode ouvert à tous, d'autant que nous ne traiterions que les questions de niveau universitaire et relevant des domaines disciplinaires de Lyon 1. Deuxièmement, le statut de CADIST Chimie et Pharmacie du SCD Lyon 1 impliquait que des étudiants d'autres universités viendraient taper à notre porte pour des renseignements documentaires dans ces domaines, il convenait donc qu'ils puissent le faire.

\section{LE TYPE DE QUESTION ATTENDU}

Trois domaines devaient être couverts :

- pratique : sur le fonctionnement des BU de Lyon 1 et leur organisation ;

- documentaire : pour trouver une référence, démarrer une bibliographie, utiliser une base de données, etc. ;

- informatique : sur l'utilisation et la connexion aux postes en libre accès à la BU, la connexion au Wifi, l'accès à la documentation électronique, etc.

Le type de réponse visé : nous ne cherchions pas l'exhaustivité : il s'agissait de répondre de façon pédagogique, en proposant un parcours de recherche mettant en avant les outils documentaires de la BU.

Le délai de réponse : une question pouvait être posée à n'importe quel moment, mais le service n'était ouvert que du lundi au vendredi et hors périodes de fermeture des BU de Lyon 1 . La question était traitée dans les 48 heures ouvrées suivant sa réception.

\section{QUELLE ORGANISATION ?}

Les bibliothécaires de Lyon 1 craignaient de devoir affronter un surcroît de travail important. Or, pour un REL de BU, le nombre de questions posées 
est en général d'une dizaine à une cinquantaine par mois et la charge de travail par personne se révèle peu importante. Par ailleurs, il existait déjà au SCD un système de plages de renseignement aux banques de prêts sur lequel on pouvait s'appuyer. 3 propositions d'organisation ont été faites aux membres du groupe de travail REL :

- proposition 1 : les catégories A et B répondent aux questions qui arrivent durant leurs plages de permanence. Ils peuvent ne pas y répondre tout de suite mais les questions arrivées durant cette période leur sont rattachées ;

- proposition 2 : nommer des responsables disciplinaires qui répondront aux questions de leur domaine de compétence ;

- proposition 3 : constituer un pool santé et un pool science, chacun de 3 à 5 personnes, toutes catégories confondues, qui se partageront les réponses.

La solution 3 a été adoptée, la participation se faisant sur la base du volontariat. 5 catégories de questions, correspondant à 5 groupes de "répondants », ont été constituées.

Les groupes :

- renseignements pratiques BU Sciences ;

- renseignements pratiques BU Santé ;

- renseignements en documentation électronique et informatique ;

- renseignements bibliographiques dans les domaines des sciences et du sport ;

- renseignements bibliographiques dans les domaines de la santé. À ces 5 groupes de 4 à 7 participants s'ajoutaient 2 modératrices.

\section{LORSQUE LE REL ÉTAIT SUR LE SITE WEB}

Les modératrices étaient en copie systématique des questions et réponses échangées. Leur rôle était de vérifier que les questions posées avaient bien reçu une réponse dans les délais et de constituer un fichier recensant toutes 
les questions posées et les réponses données, classées de façon thématique, afin de constituer une base de connaissances. Les 5 groupes étaient dotés de 5 adresses génériques. En fonction du type de question posée (à cocher par l'utilisateur), cette dernière était automatiquement envoyée au groupe correspondant. Tous les membres du groupe et les modératrices recevaient la question. Sa prise en charge se faisait librement : celui qui désirait répondre envoyait un message à l'ensemble de son groupe en précisant « je m'occupe de cette question ». Il avait alors 48 heures pour répondre à l'usager en mettant en copie les modératrices.

\section{LORSQUE LE REL EST PASSÉ SUR LE PORTAIL}

Le back office a basculé sur l'interface du logiciel INEO et le fonctionnement en a été modifié, mais il est resté le même pour l'usager (remplir un formulaire). L'ensemble des participants recevait une notification dans sa messagerie pour chacune des demandes, avec indication du groupe concerné et un lien vers le back office. À chaque arrivée, la modératrice vérifiait le contenu de la demande et intervenait si nécessaire pour réorienter la question. Chaque membre du groupe concerné était libre de choisir de répondre ou non, et un workflow se mettait alors en place. Toutes les questions et réponses étaient conservées dans le système pour servir de base de connaissances. Une FAQ était également proposée aux usagers.

À ce jour, le service fonctionne bien et les groupes sont rodés. Ils ont répondu à 280 questions en 2008 et 210 en 2009 . 


\section{COMMENT PROMOUVOIR UN
SERVICE DE RENSEIGNEMENT \\ COMMENT PROMOUVOIR UN
SERVICE DE RENSEIGNEMENT EN LIGNE?}

par Nicolas Alarcon

\section{4}

Nous ne renseignons plus nos usagers comme il y a 15 ans. Internet est passé par là, en bouleversant autant nos pratiques de renseignements que les pratiques de recherche et les attentes de nos publics. Alors, pourquoi la promotion de nos services de Q/R n'a-t-elle pas suivi la même (r)évolution ? L'existence et le rôle du bureau de renseignements seraientils une évidence pour nos publics? D’ailleurs, que représente un bureau d'accueil/information pour un étudiant de la génération $\mathrm{Y}^{1}$, pour qui Internet et Google ont toujours existé ? Le marketing des services de Q/R est un champ encore peu exploité en France, c'est aussi une formidable occasion de faire basculer la promotion de l'activité renseignement des bibliothèques dans le XXI ${ }^{\mathrm{e}}$ siècle.

\section{PROMOUVOIR UN SERVICE DE RENSEIGNEMENT VIRTUEL, POURQUOI ?}

La promotion d'un service, quel qu'il soit, peut d'abord se justifier par sa nouveauté. Il convient de l'inscrire dans l'ADN de l'établissement, de le rapprocher ou de le distinguer des services existants, de le placer durablement dans le champ de vision de l'usager et de créer, dans le meilleur des cas, un noyau d'habitués assurant, par là même, un usage régulier.

La création d'un service de renseignement en ligne collaboratif (c'est-àdire regroupant plusieurs bibliothèques) nécessite un investissement de départ conséquent aussi bien financier, qu'en temps et en ressources humaines employés pour monter le projet et le gérer quotidiennement : formation des répondants, organisation des équipes, gestion des plannings, mise en place technique, évaluation, etc. En visant l'augmentation de la

1. Génération Y : terme utilisé pour désigner les jeunes d’une classe d'âge, nés entre 1979 et 1994 . On parle aussi de digital natives ou net generation. 
fréquentation du service, la promotion est l'un des moyens d'assurer un minimum de retour sur cet investissement.

Enfin, la promotion du service de renseignement en ligne permet de le pérenniser dans un environnement qui pourrait devenir concurrentiel à court ou moyen terme.

Les services de Q/R académiques se sont récemment multipliés. 2009 a vu l'ouverture de deux services collaboratifs regroupant plus d'une trentaine de bibliothèques ou SCD, sans compter les services spécifiques à un établissement d'enseignement supérieur. Or, même si ces services répondent en priorité aux membres de la communauté universitaire qu'ils desservent, ils sont généralement ouverts à tous. Les utilisateurs ont donc une large palette de services à leur disposition. Leur choix pourrait, légitimement, se porter sur un autre service que celui de leur université, selon la qualité ou le délai des réponses, mais avant tout en fonction de la visibilité dudit service dans leur environnement proche.

Une étude menée par Lynn Connaway, Marie Radford et Timothy Dickey sur le non-usage des services de renseignement physiques et virtuels a montré que la première raison de leur non-utilisation était l'absence de connaissance de leur existence. Il faut d'abord savoir qu'un service existe avant de l'utiliser ; la promotion vise cet objectif en premier lieu.

Il n'existe pas encore de réelle concurrence entre service de référence virtuel (SRV) universitaires, ni même entre SRV universitaires et autres services de renseignement de bibliothèque (BiblioSésame, SINDBAD). La concurrence pourrait provenir plutôt de projets libres ou commerciaux ${ }^{2}$ qui ont en commun leur puissance et leur dimension communautaire : les usagers du service répondent à d'autres usagers. Par exemple, l'Oracle ${ }^{3}$, service de renseignement du Wikipédia francophone, ouvert en 2005, recueillait à son acmé plus d'une centaine de questions par semaine. Une récente étude sur la version anglophone de l'Oracle (Reference Desk) conclut même que la qualité des réponses produites par le service de Wikipédia est comparable à celle des services de bibliothèques.

2. Cf. l'article de C. Nguyen, « Typologie des services de questions-réponses », p. 146.

3. L'Oracle : < http://fr.wikipedia.org/wiki/Wikip\%C3\%A9dia:Oracle > (consulté le 3 mai 2010). 
Après une tentative avortée en 2004, Google compte réinvestir ce « marché » après le rachat en février 2010 de la société Aardvark et de son site communautaire de renseignements. Le leader incontesté reste Yahoo Questions/Réponses, service aux statistiques vertigineuses ${ }^{4}: 1$ milliard de questions/réponses postées dans le monde, 179 millions de visiteurs uniques, 15 millions d'utilisateurs quotidiens. Yahoo Q/R tente de nouer des partenariats avec des sociétés privées ou des organisations publiques. Enquire (service de Q/R en ligne britannique est un contributeur actif depuis 2007 et jouit d'une très bonne réputation parmi les participants du site anglais). Ces sites sont déjà ou seront prochainement utilisés par nos usagers. Face à ces sites, si les bibliothèques veulent s'engager et perdurer dans ce secteur, elles devront jouer, sans complexe, la carte du marketing afin de se positionner auprès de leur public et de se singulariser par rapport aux services existants.

\section{IDENTIFIER LES PUBLICS, MOBILISER LES RELAIS}

Avant de mettre en place la campagne de promotion, il convient de déterminer à qui elle s'adressera en priorité. En effet, l'orientation du message et les modalités de la communication (supports, fréquence, lieu) différeront selon que l'on s'adresse à des étudiants de niveau Licence, des publics empêchés, des doctorants ou des enseignants-chercheurs. Un choix s'impose, une communication visant trop large risque de rater toutes ses cibles. L'e-mail, par exemple, est en perte de vitesse chez les plus jeunes publics des BU ; comme vecteur de communication, on pourra lui préférer les réseaux sociaux (une page de fans Facebook par exemple), très fortement utilisés par cette tranche d'âge. Le chat est une pratique courante des étudiants à l'heure actuelle ; en insistant sur le renseignement par messagerie instantanée dans une campagne, on tirera avantage d'une activité immédiatement identifiable par le public-cible.

4. Service sur < http://fr.answers.yahoo.com/ > (consulté le 3 mai 2010). Données au 15 octobre 2009 issues du blog Yahoo answers < http://www.yanswersblogfr.com/b3/2009/10/05/le-saviez-vous > (consulté le 3 mai 2010). 
Si les primo-arrivants représentent les usagers prioritaires pour le SRV, la promotion doit débuter dès les premières visites de la bibliothèque (voire, à la rentrée, lors de la présentation de l'ensemble des services universitaires), se poursuivre lors des séances initiales de formation à la recherche documentaire et être distillée chaque fois que nécessaire aux bureaux d'accueil et de renseignement.

Les enseignants-chercheurs pourront être approchés par des méthodes plus officielles, courrier papier et plaquettes de présentation adressés directement ou avalisés par les responsables de département et les directeurs de laboratoires. Les enseignants-chercheurs représentent d'ailleurs un double enjeu. À la fois public potentiel du service, ils constituent également d'excellents relais de communication auprès d'une autre cible, les étudiants. Les actions marketing dans leur direction devront prendre en compte ce paramètre.

Une campagne promotionnelle doit savoir s'appuyer sur des réseaux de personnes participant à la diffusion du message proposé. Par leur proximité avec les étudiants, les moniteurs employés par la bibliothèque sont en mesure de relayer l'information sous une forme moins institutionnelle et prescriptive que les enseignants-chercheurs, plus proche de la recommandation par les pairs.

Les bibliothécaires doivent rester, néanmoins, les principaux relais du service auprès des publics. Si les SRV s'appuient généralement sur une équipe réduite de volontaires, la promotion sera plus efficace si elle est assurée par l'ensemble des personnels. Tous les bibliothécaires devraient être régulièrement informés de l'évolution du service par des présentations et des démonstrations. Ils seront alors à même de le promouvoir activement en situation d'accueil et de renseignement.

\section{SOIGNER SON IMAGE DE MARQUE}

Un service de renseignement virtuel n'est pas un concept immédiatement compréhensible par l'usager, l'image de la bibliothèque véhiculant encore souvent les mêmes poncifs : prédominance du livre papier, vétusté, manque de réactivité. Pour y remédier, le service doit créer, diffuser et entretenir une véritable image de marque auprès de ses publics. 


\section{Qu'est-ce qu'une marque ?}

C'est un nom, un logo, un symbole qui, en identifiant une organisation, ses biens et ses services permet de se distinguer de ses concurrents.

Le contrôle (même partiel) sur l'image perçue par les usagers facilitera la communication entre le ou les prestataires du service et les publics. Le nom doit être le plus explicite possible quant à la fonction du service et la marque gagnera à être associée à sa principale caractéristique à l'aide d'un slogan : "Vous avez une question ? Nous avons la réponse ! (Le Guichet du savoir), « Vos bibliothécaires en ligne » (Ubib.fr), « Un réseau de bibliothécaires à votre écoute » (BiblioSésame). Les bibliothèques académiques nord-américaines emploient généralement le même nom pour désigner les SRV : Ask a Librarian ou Ask us. La clarté et la répétition de l'expression en font une marque descriptive et immédiatement identifiable par les usagers. En France, le nom Questions ? Réponses !

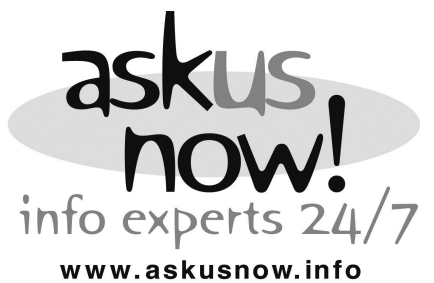

Logo du service collaboratif AskUsNow! (Maryland, États-Unis) est utilisé par quelques services mais ne s'est pas imposé aussi largement. L'arrobase (@) dans un nom de service est une pratique datée qui peut poser des problèmes de référencement aux moteurs de recherches, le logogramme étant principalement utilisé pour les adresses de courrier électronique.

Le nom de la marque sera associé à un élément visuel qui rendra sa mémorisation et sa diffusion sur les supports de communication plus aisées. Ce logo pourra se décliner au gré des campagnes de promotion et des supports employés. Il évoluera également en fonction des tendances et des publicscibles. Les compétences en matière de graphisme et de design étant relativement rares dans les bibliothèques, il ne faudra pas hésiter à faire appel à des professionnels ou mobiliser les filières créatives de l'université. Le coût d'une telle opération sera rapidement rentabilisé en termes d'image du service. 
Une fois le nom et le logo créés, certaines opérations permettront, à moindre coût, de maîtriser sa marque et d'avoir le contrôle total de sa diffusion. Si l'on souhaite préserver l'originalité d'un nom et d'un logo, il est possible de les déposer auprès de l'Institut national de la propriété intellectuelle (INPI). Le coût varie en fonction du nombre de classes (catégories d'activité) dans lesquelles la marque sera déposée mais il reste raisonnable (moins de 400 euros pour 10 ans).

La création d'un site Web propre au service de Q/R augmentera significativement sa visibilité sur les moteurs de recherche mais peut, néanmoins, engendrer une désaffiliation entre le service et le ou les établissements qui y participent au cas où la marque du service supplante celle de la bibliothèque. Pour les bibliothèques universitaires, le dépôt du nom de domaine, étape obligatoire de la création d'un site, peut s'effectuer gratuitement via le réseau RENATER qui assurera également le renouvellement du dossier.

\section{MULTIPLIER LES SUPPORTS}

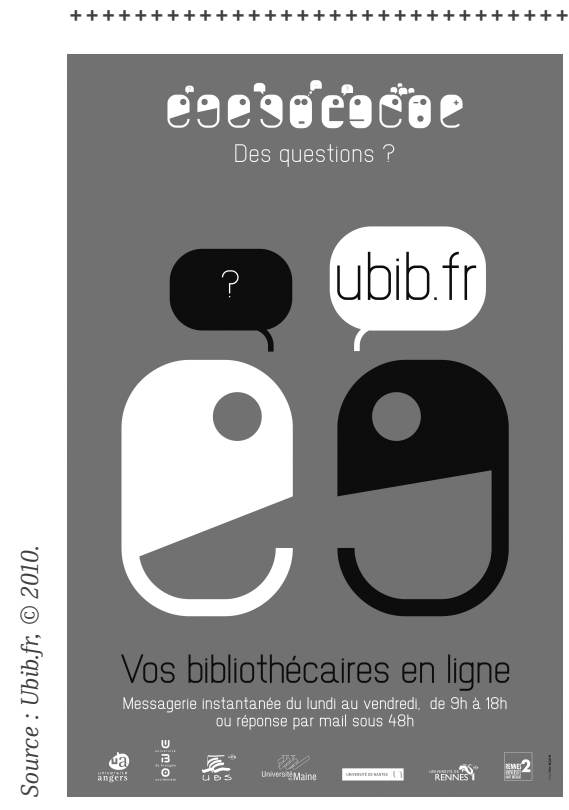

Affiche $40 x 60$ du service en réseau Ubib.fr
Les affiches publicitaires (sur papier ou sur écran) restent un moyen traditionnel mais efficace de faire connầtre l'existence et le rôle d'un service de Q/R. Les salles des bibliothèques seront un espace privilégié d'affichage : à l'accueil, dans les espaces informatiques, à proximité du bureau de renseignement. Néanmoins, une campagne de promotion doit aussi et surtout se jouer hors les murs. Pour les bibliothèques académiques, les espaces de l'université doivent être investis tout en conservant la maîtrise de sa communication. Les espaces d'affichage libre sont donc à éviter, les affiches ne resteront pas assez de temps en place pour être vues par le 
public. Les services universitaires peuvent être de précieux alliés dans cette recherche de visibilité, ils disposent de zones d'affichage contrôlées très fréquentées des étudiants : panneaux d'affichage des scolarités, cités et restaurants universitaires.

Pour accompagner l'usager dans son quotidien, la marque doit également se décliner sur d'autres supports. Les incitatifs ou goodies* sont des objets de la vie courante frappés du logo d'une marque : stylos, pots à crayons, magnets, bloc-notes, stickers, etc. Distribués en formation, aux bureaux

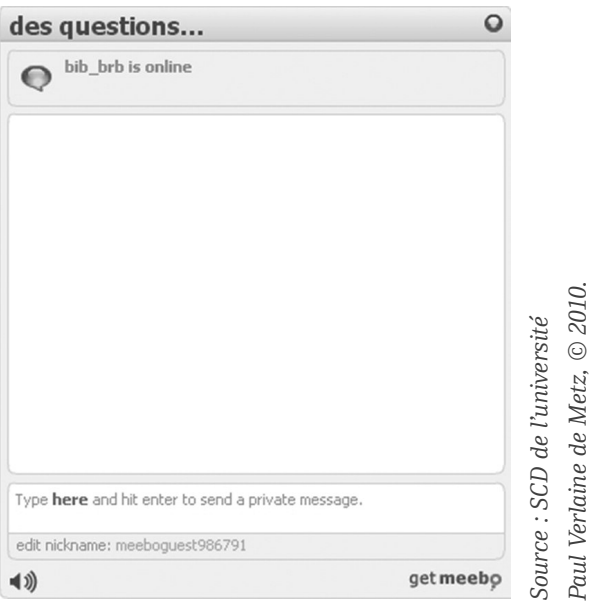

Widget Meebo utilisé par le SRV de l'Université de Metz d'accueil ou à toute autre occasion, ils entreront dans l'environnement du public tout en dépassant le simple marque-page qui perd de son sens pour un service en ligne.

La création de supports de communication a un coût qui peut rapidement augmenter en fonction du type choisi et de la qualité de fabrication. Dans le cas du service en réseau Ubib.fr, l'impression de 1000 affiches, 20000 marque-pages et la fabrication de 10000 crayons et 200 pots à crayons a coûté près de $4700 €$ TTC répartis entre les sept universités participantes.

\section{MARQUER SA PRÉSENCE EN LIGNE}

La meilleure des promotions, et la moins coûteuse, est la multiplication sur le Web du lien vers le service. Si les affiches et les goodies requièrent une présence physique de l'usager, un service de Q/R doit tirer parti de sa dimension virtuelle. Au-delà de la simple promotion, c'est aussi offrir la possibilité d'utiliser le service en un clic, là où l'usager se trouve et pas seulement sur les pages Services ou Renseignements du site de la bibliothèque.

Les SRV proposant la messagerie instantanée peuvent désormais offrir plus qu'un simple lien vers le service. Grâce à une fenêtre facilement importa- 
ble dans un site Web (un widget*) et permettant de " discuter » avec un bibliothécaire, le service devient potentiellement accessible partout sur internet. La disponibilité immédiate du service devient sa propre promotion. La dissémination du widget ${ }^{5}$ là où se trouve l'usager constitue l'action marketing la plus prometteuse pour valoriser un SRV et assurer sa fréquentation. Cette option a également l'avantage de correspondre aux pratiques numériques des étudiants, la fenêtre de chat (MSN, Meebo, Gmail, Facebook) fait pleinement partie de leurs outils de communication quotidiens.

Sur les sites de bibliothèque, le lien, matérialisé par le nom, le logo ou le widget devrait être proposé dès la page d'accueil et sur chaque page pour que l'internaute puisse y accéder quand il en a besoin. La page Renseignement, moins consultée, pourra présenter en détail tous les modes d'interrogation proposés (e-mail, chat, vidéo, téléphone, bureau physique, fax).

Les sites internet " institutionnels " fréquentés par les étudiants devront être massivement investis avec l'accord des services responsables ${ }^{6}$ : environnement numérique de travail (ENT), plateforme de cours en ligne, catalogue informatisé (lorsqu'aucun résultat n'est trouvé par exemple), blog de bibliothèque, etc. Comme pour les affiches, la promotion en ligne doit déborder des espaces gérés par l'université ou la bibliothèque sans toutefois perdre le contrôle de son image ou du message diffusé. Par son taux de pénétration dans nos publics, le réseau social Facebook n'est pas à négliger.

Tout nouveau service de Q/R se dotera d'une stratégie marketing ambitieuse s'il souhaite s'inscrire durablement dans l'environnement numérique des usagers. L'installation d'une nouvelle marque est un processus long et parfois coûteux ; c'est néanmoins le meilleur moyen d'amorcer et de consolider l'activité minimum qui assurera la pérennité du service. Enfin, il faut garder à l'esprit qu'en matière de promotion, tout ce qui est bon pour le service de $Q / R$ est bon pour la bibliothèque en général.

5. Cf. l'article d'Hélène Tardif, « Quels outils pour quels services ? Quelle technologie choisir ? », p. 94.

6. Cf. l'article de J.-C. Houpier, « Service de questions-réponses et environnement de l'usager : quelle intégration ? », p. 59. 


\section{5}

SERVICE DE QUESTIONSpar Jean-Charles Houpier RÉPONSES ET ENVIRONNEMENT DE L'USAGER : QUELLE INTÉGRATION ?

\section{L'ENVIRONNEMENT DE L'USAGER}

Les «publics » sont incontestablement, des « dévoreurs » d'espaces Web, convaincus d'y dénicher l'information très rapidement. Cette première notion d'immédiateté et de fonctionnement " au plus proche » dans la recherche d'information est très importante. Elle conditionne fortement la réussite d'un service de questions-réponses, autant pour les aspects ergonomiques que pour les aspects fonctionnels. L'usager utilise les outils au gré de ses recherches, mais il sait pertinemment distinguer ceux qui répondent le mieux à son mode de fonctionnement et de raisonnement ; ils deviennent rapidement les compagnons de ses usages courants. Dans le cadre de l'utilisation d'un service de $Q / R$, il va être exigeant sur les délais de réponse ainsi que sur la qualité des réponses. L'environnement de l'usager est également conditionné par les aspects visuels, proprement subjectifs. Il « converse » plus aisément avec une interface dépouillée, claire et très facile à appréhender. Cette interface doit incontestablement offrir le maniement le plus intuitif possible.

\section{En résumé}

L'usager n'est pas novice, il a une pratique certaine des outils de demande de renseignements dans des environnements différents (sites de l'administration publique, sites de vente en ligne...). Il faut lui proposer un outil de niveau équivalent en termes de qualité ergonomique et fonctionnelle. 


\section{QUELLES FONCTIONS ?}

La mise en place d'un service de $Q / R$ suppose une réflexion préalable afin de cibler les objectifs que l'on souhaite atteindre, car les possibilités techniques sont nombreuses, tant sur le plan du questionnement que de la réponse (cf. chapitre 2).

La question peut être posée soit à l'aide d'un texte, d'un contact audio ou d'un contact audio/vidéo.

De nombreux établissements font le choix de proposer un éventail d'offres graduées afin d'être en mesure d'apporter des réponses et du service à un maximum d'utilisateurs, quelles que soient les conditions matérielles mises en œuvre. La bibliothèque de l'université de Cornwell a proposé un service de type visioconférence (CU-SeeMe) dès 1997.

\section{En résumé}

Plus l'offre et les possibilités de contact seront multiples, plus l'efficacité du service de questions-réponses sera valorisée et plus les questions d'intégration de ces services se posent.

\section{QUELS FORMULAIRES POUR L'UTILISATEUR ?}

Il est important de bien définir les éléments indispensables et strictement nécessaires pour mettre en place un service de Q/R. L'élément le plus trivial permettant d'identifier simplement un utilisateur, et surtout d'être en mesure de lui adresser une réponse, est son adresse e-mail. C'est une information qu'il est demandé de saisir à l'utilisateur. Peu de moyens de contrôles peuvent être mis en place, l'idéal serait d'adosser cette zone à un annuaire. Pour éviter les erreurs dans la frappe de l'e-mail, on peut proposer une confirmation - en obligeant le lecteur à ne pas faire de simples copier/coller!

Il faut considérer que le public, qui désire utiliser un service en ligne synchrone (téléphone, messagerie instantanée) ou asynchrone, n’a pas particulièrement envie de fournir de nombreux éléments d'identification avant 
de poser sa question. Le professionnel des bibliothèques est, quant à lui, soucieux de connaître l'origine des publics qui utilisent le service, et il est tenté de demander à l'utilisateur de multiples renseignements, tels que : sa faculté d'origine, son niveau d'étude, sa localisation géographique, le code postal de sa ville d'hébergement, le pays d'origine, sa tranche d'âge, les bibliothèques fréquentées, le délai de réponse souhaité, l'objectif de la demande, l'accord sur l'archivage de la demande, les ressources utilisées... Attention aux choix par défaut (exemple : mettre France en haut du formulaire, et pas Arabie Saoudite). Par contre, une question sur l'objectif visé est très importante pour orienter la réponse. Dans la même logique, on peut également lui demander de choisir la thématique de sa question ${ }^{1}$.

Dans le cas d'un échange basé sur une correspondance écrite, l'interface devra être composée d'au minimum 2 zones :

- une zone permettant la saisie de l'e-mail ;

- une zone de saisie de la question de taille bien proportionnée.

\section{Exemple du formulaire du service de $Q / R$ de la вpi}

< http://www.bpi.fr/fr/la_bpi_et_vous/questions_reponses/posez_votre_ question.html >

La question de la taille de la zone de saisie est à considérer avec soin afin que visuellement l'utilisateur ne se sente pas «freiné » ou a contrario « noyé » par des dimensions mal appropriées.

Exemples de formulaire de « messagerie instantanée » de bibliothèques américaines

< http://www.med.yale.edu/library/services/crs/chat.html > (Université de Yale)

< http://www.lib.umich.edu/ask-librarian > (Université du Michigan)

Dans le cas d'un échange basé sur une conversation audio ou audio/vidéo, un $\operatorname{logo}^{2}$ devra expliciter clairement la nature du service, ainsi que les outils nécessaires pour pouvoir dialoguer.

2. Exemple de logos : < http://lib.asu.edu/help > (Université de l'Arizona). 


\section{En résumé}

L'aspect visuel de la présentation du service est extrêmement important sur deux plans :

- identification claire du service proposé ;

- appropriation de l'interface de dialogue par l'usager.

\section{INTÉGRATION DU SERVICE DE Q/R À L'ENVIRONNEMENT DE L'USAGER}

L'intégration du service de questions-réponses aux outils de l'établissement répond à un souci de fluidité de la navigation de l'utilisateur. Il est utile de retenir celui-ci dans un environnement de même qualité visuelle et ergonomique, ce qui aura pour effet de le conforter dans sa perception de l'institution lui fournissant des outils et des réponses.

\section{INTÉGRATION AU SITE WEB OU PORTAIL}

Le site Web de l'institution est le premier point de rencontre avec le public. C'est aussi sur les sites Web des établissements que le service de questionsréponses est le plus souvent proposé. À cet égard, tous les degrés d'intégration sont mis en œuvre, et nous proposons d'en lister brièvement les modalités.

- La «non-intégration » qui est caractérisée par le renvoi vers un site externe qui peut être un service de questions-réponses collaboratif ou un service assuré par une plateforme gratuite sur le Web. C'est souvent le cas pour les outils de messagerie instantanée (Meebo, Imo.im, Tinychat), qui n'ont pas de sens à être développés en interne.

- L'intégration à l'aide d'un widget ${ }^{3}$. Cette solution est correcte sur le plan visuel bien qu'elle ne réponde pas parfaitement à la charte graphique du site.

- L'intégration à l'aide d'une API XML* est certainement la meilleure solution, car visuellement la mise en

3. Cf. les articles d’H. Tardif «Quels outils pour quels services ? Quelle technologie choisir ?», p. 94 et de N. Alarcon « Comment promouvoir un service de renseignement en ligne ?», p. 51. 
œuvre de la charte graphique est complète. Mais pour des solutions simples, comme un formulaire, l'intégration se fait à l'aide de quelques lignes de code HTML*.

L'intégration des outils d'un service de Q/R comme le formulaire d'envoi d'e-mail, la messagerie instantanée, la visioconférence est de plus en plus proposée par les fournisseurs de services eux-mêmes.

\section{INTÉGRATION À L'ENT}

L'environnement numérique de travail (ENT) est pour l'étudiant le lieu privilégié d'accès aux ressources et aux services de l'établissement. Plutôt que de regrouper les services de la documentation dans un onglet, il est de loin préférable de disséminer ces mêmes services dans les fonctions majeures proposées par l'ENT. Ainsi, la plateforme ESUP Portal utlisée par plus de 80 établissements universitaires en France, est constituée de plusieurs modules assurant entre autres l'authentification, la communication, le stockage, la scolarité, et la publication (CMS). Dans la partie communication, on trouve un outil « Helpdesk » qui assure l'aide aux utilisateurs au travers d'un outil de gestion des questions-réponses. Ce service peut être amélioré par la création d'une passerelle entre le formulaire situé sur le site Web et le « Helpdesk » de l'ENT. Ce travail a été réalisé par le CRI de l'université Henri-Poincaré (UHP). La gestion des questions-réponses en provenance soit du formulaire du site Web, soit de l'ENT, est assurée par un système unique. Ce module d'interfaçage est bien sûr mis gracieusement à disposition de la communauté ESUP. En résumé, l'objectif reste en permanence d'utiliser au mieux les ressources habituelles des usagers en adaptant les workflows et en visant une intégration maximale.

\section{INTÉGRATION AU RÉSOLVEUR DE LIENS}

Le résolveur de liens* propose des services contextuels au niveau d'une référence bibliographique tels que l'accès direct au texte intégral, l'accès à la référence bibliographique du document ou à sa version imprimée, l'accès à des ressources et services complémentaires. 
L’intérêt de proposer ce service au niveau du résolveur de liens permet de répondre à toutes les questions d'accès à la ressource électronique. Il est difficile de proposer des exemples consultables directement, puisque cette fonction n'apparaît qu'après authentification. Malgré tout, je citerai le résolveur de liens de l'université Paris Descartes qui propose le service sous l'appellation " Poser une question à un bibliothécaire ».

Exemple de la Bibliothèque Interuniversitaire de pharmacie - Paris Descartes < http://www.biup.univ-paris5.fr/resolveur.html >

\section{En résumé}

Le service de questions-réponses peut être proposé au niveau le plus fin de la recherche documentaire, c'est-à-dire au niveau du document primaire.

\section{INTÉGRATION AUX RÉSEAUX SOCIAUX}

Les réseaux sociaux sont des éléments prépondérants des possibilités d'interaction des utilisateurs avec les applications du Web, on parle alors d'applications Web 2.0. Facebook, Twitter, MySpace, LinkedIn sont les chefs de files actuels des outils de ce type. Ils participent à l'objectif de dissémination des services de la bibliothèque sur le Web. Il ne s'agit pas de faire intrusion dans la vie des utilisateurs, mais bien d'étendre la présence de la bibliothèque. Leur intérêt majeur n'est pas de proposer de nouveaux services, mais de proposer les services existants (catalogue, renseignement en ligne...) là où sont les utilisateurs. En 2005, la bibliothèque du Brooklyn College fut la première à proposer ce service sur MySpace.

\section{Exemples des Bu d'Angers et de Marseille III}

< http://www.facebook.com/pages/Angers-France/Bibliotheque-BelleBeille-Universite-dAngers/270807194287?v=app_7146470109 > < http://www.facebook.com/budroitaix?v=box_3 > 
Le site Facebook de la BU de l'université Paul Cézanne est le plus abouti, car il propose une étagère de nouveautés, et surtout un widget MeeboFB pour le service de renseignement en ligne.

L'autre grande richesse de ces outils tient à leur interopérabilité et à leur interconnexion de base. Pratiquement, des informations publiées sur l'un sont immédiatement visibles sur l'autre, ce qui est le cas pour tout ou partie de celles-ci : Facebook, Twitter, MySpace, Meebo, imo.im, Yahoo Messenger, YouTube, MSN, GTalk, Skype, Gmail, TweetDeck, Delicious, GoogleReader... Leur parfaite intégration permet à l'utilisateur de naviguer dans un environnement intégrant parfaitement les outils les plus habituels, et de passer de l'un à l'autre sans difficulté.

En résumé, l'intégration du service de $Q / R$ aux réseaux sociaux permet d'atteindre deux objectifs :

- liens personnalisés avec un grand nombre potentiel d'utilisateurs ;

- intégration des outils de base de l'utilisateur aux services proposés.

Prendre garde à ne pas « spammer » le réseau d'amis constitué, car chaque modification du profil est reçue par chaque membre.

\section{INTÉGRATION À UN BLOG}

L'intégration à un blog résulte d'une volonté affichée de mettre l'utilisateur au centre des préoccupations et surtout de conserver toute sa crédibilité à la bibliothèque souhaitant mettre en place une médiation numérique de qualité avec son public. Le blog est un outil familier et participatif du Web 2.0 qui permet de stocker et d'organiser de l'information de manière chronologique, alphabétique, thématique, systématique tout en apportant une possibilité d'interaction et de dialogue. Tout le travail consiste à adapter les fonctionnalités « naturelles » du blog à un service de Q/R. 
En cela, l'étude ${ }^{4}$ d'opportunité d'un blog pour SwissInfoDesk (Bibliothèque nationale Suisse) basée sur une volonté de rendre publiques les questionsréponses est intéressante. Cette étude démontre la faisabilité d'un tel projet et surtout sa facilité de mise en œuvre.

\section{Les points remarquables qu'offre cette solution}

Suivre les questions posées sur une thématique donnée (flux RSS*), mesurer les domaines où les questions sont les plus nombreuses, possibilité de ne pas publier certaines questions-réponses, améliorer le référencement, utiliser le blog comme base de connaissances pour les formations, insertion de médias (vidéos, documents sonores...) directement dans la réponse.

Les améliorations que l'on peut entrevoir : intégrer le formulaire permettant de poser une question, implémenter le plug-in permettant de suggérer les questions-réponses ayant une même indexation (articles relatifs), possibilité de limiter l'accès de ce service par authentification aussi bien aux utilisateurs qu'aux personnes chargées d'animer ce service, multilinguisme, mettre en valeur des thématiques grâce au flux de données correspondant.

Le site de l'American Library Association a mis en œuvre son service de Q/R sous cette forme :

$<$ http://americanlibrariesmagazine.org/askthelibrarian >

\section{En résumé}

Le blog est un outil aux multiples possibilités, très facile à mettre en œuvre, que l'on peut tester sans limites avant sa mise en production, pour atteindre des objectifs aussi variés que de proposer un service de $Q / R$, un site de nouvelles acquisitions, un outil de communication interne ou externe... Le blog a l'avantage de proposer ces diverses fonctionnalités dans un environnement parfaitement intégré et autorisant une « re-publication » par flux RSS dans une messagerie, un site Web, un agrégateur, des écrans...

4. Reginelli Marie. Archivage et publication en ligne des questions/réponses d'un service de référence à distance. Étude d'opportunité d'un blogue pour SwissInfoDesk. CESID - Diplôme universitaire de formation continue en information documentaire, Université de Genève, sept. 2009 (non publié). 


\section{INTÉGRATION À UNE PAGE WEB POUR MOBILES}

Apporter le service de Q/R au niveau d'un smartphone (en dehors de la conversation téléphonique) peut s'envisager sous forme d'échanges de SMS. Les bibliothèques ayant mis en œuvre ce service ont eu la volonté de l'intégrer au workflow du service de Q/R existant. De l'avis des bibliothèques l'ayant adopté, ce service ne génère pas encore énormément de questions, mais elles tiennent absolument à le conserver, car elles supposent que son expansion est à venir.

Actuellement, des applications sont développées pour l'iPhone spécifiquement par les bibliothèques et disponibles sur l'Apple store : Worldcat FR Mobile (catalogue OCLC), DCPL (catalogue des bibliothèques publiques de Washington, horaires d'ouverture et géolocalisation).

L'idéal serait de développer des applications de questions-réponses spécifiquement pour les smartphones en ayant le souci de les intégrer dans le workflow des réponses.

\section{EN CONCLUSION}

Le choix de «peaufiner » l'intégration de l'outil de questions-réponses résulte plus d'une démarche, en direction de l'utilisateur, afin de l'amener dans un environnement intuitif et agréable à utiliser afin de lui donner, tout simplement, l'envie de revenir. 


\section{6}

VALORISER LE SERVICE DE QUESTIONS-RÉPONSES ET L'INTÉGRER AUX AUTRES SERVICES DE LA BIBLIOTHĖQUE

Le Guichet du Savoir < http://www.guichetdusavoir.org >

La plupart du temps, un service de questions-réponses repose sur l'intimité d'un colloque singulier entre un usager et un bibliothécaire. Ce faisant, il apparaît comme détaché des autres services de la bibliothèque, qui reposent sur des prestations moins confidentielles. La situation est très différente dans le cas où questions et réponses sont publiées, car l'échange interindividuel s'expose et prend un caractère public affiché1. Quelques services en ont pris le parti, comme Le Guichet du Savoir (GdS) de la BmL. C'est sur ce dernier exemple que je m'appuierai essentiellement pour évoquer l'intégration et la valorisation du service de $Q / R$ au sein des autres services et même au sein de l'offre globale de contenus de la bibliothèque.

\section{LA VALORISATION DES BIBLIOTHÉCAIRES}

Précisons pour commencer que les réponses apportées sur Le GdS sont rédigées pour partie par une équipe dédiée de trois bibliothécaires, appuyée sur l'ensemble des professionnels des départements thématiques de la bibliothèque de la Part-Dieu et de deux « départements » spécialisés des deux médiathèques du réseau. C'est sur l'expertise en contenus de ces professionnels que le pari du GdS s'est donc appuyé2.

Rédiger des réponses lisibles par tous, ce n'est pas seulement s'efforcer de satisfaire l'interrogation d'un interlocuteur, c'est aussi s'exposer, car c'est vraiment publier. Le bibliothécaire est alors jugé à plusieurs titres :

1. J'entends par « affiché » le libre butinage offert aux internautes qui voudraient feuilleter les réponses, et non un moteur de recherche qui ne sélectionnera dans une base de connaissances - souvent étanche aux moteurs de recherche - que les seuls résultats qu'on aura pu accepter d'y voir apparaître.

2. Voir la contribution de C. Di Pietro, «Constuire un service de référence généraliste : l'exemple du Guichet du Savoir de la Bibliothèque municipale de Lyon », p. 152. 
- les bibliothécaires, peu habitués à être placés ainsi sur le devant de la scène, peuvent au début être inquiets de leur qualité de rédaction. Cela les incite à soigner leur expression, à peser les termes de leur réponse, à respecter un code d'écriture commun;

- la publication confronte également les professionnels aux réalités de la protection des droits d'auteur ${ }^{3}$;

- enfin et surtout, la publication impose d'être à la hauteur des attentes des demandeurs, et aussi des lecteurs de passage. Exposée, l'erreur ou l'approximation sera immanquablement remarquée et dénoncée, soit par des e-mails aux bibliothécaires soit par des billets assassins sur d'autres sites.

Ces exigences, qui peuvent paraître effrayantes au débutant, offrent une autre face beaucoup plus agréable. Car si le style maladroit ou l'erreur sont vite dénoncés, la réponse qui cerne bien le champ de la question, qui sélectionne les sources pertinentes et produit une réponse de bonne tenue est également vivement remarquée. Les félicitations des demandeurs, mais aussi celles de lecteurs de passage ne manquent pas d'arriver par e-mail, et dans le cas de la $\mathrm{BmL}$ on ne compte plus les références à telle ou telle réponse du GdS dans des forums ou sites spécialisés, et jusque dans Wikipédia ${ }^{4}$ ! Cette reconnaissance publique est sans aucun doute la meilleure valorisation non seulement du service mais aussi et surtout des bibliothécaires, qui se sentent ainsi confortés et reconnus dans leur expertise.

\section{INTÉGRER LE SERVICE DE QUESTIONS-RÉPONSES DANS L'ACTIVITÉ DE LA BIBLIOTHĖQUE}

Si quelques agents sont dédiés au bon fonctionnement du service, les dizaines d'autres qui apportent leur expertise par de multiples réponses

3. Voir l'article d'A. Svenbro, « Le cadre juridique des questions et des réponses », p. 92.

4. À titre d'exemple : < http://fr.wikipedia.org/wiki/Bombardement_du_26_mai_1944 > (consulté le 3 mai 2010). 
doivent composer avec la vie quotidienne de leurs activités documentaires : opérer des sélections, élaborer des conférences ou expositions, accueillir le public, etc. Le service de Q/R est dans ces conditions une charge de travail supplémentaire dont il faut organiser le flux, d'autant plus redoutable qu'il est imprévisible.

Le plus difficile n'est pas tant dans cette logistique des permanences que dans la charge de travail parfois très lourde apportée par telle ou telle question délicate, requérant parfois de nombreuses heures de recherches et de contacts.

\section{L'expérience lyonnaise montre que deux ingrédients sont nécessaires pour faire prendre la mayonnaise :}

- du côté des départements récepteurs, se convaincre que l'exercice de la réponse est un impératif de service, d'autant plus utile qu'il peut être réinvesti dans d'autres services et compétences (en assistance présentielle, en contacts de partenaires ou en production de bibliographies...) ;

- du côté de la cellule gestionnaire, veiller précautionneusement à éviter de surcharger tel ou tel département de questions redirigées, bref à réguler le flux en acceptant, dans la mesure du possible, de s'engager dans des réponses à des questions parfois spécialisées. Il ne faut jamais oublier que le service de Q/R n'est qu'un service parmi d'autres, et ne doit pas affaiblir la qualité des autres prestations...

\section{L'EXTENSION DU CHAMP DE LA BIBLIOTHĖQUE}

Les premières réponses apportées sur Le Guichet du Savoir tendaient à se référer aux ressources imprimées de la bibliothèque. Mais cette position présentait plusieurs inconvénients tant pour la rédaction des réponses (difficulté de trouver les passages pertinents, lourdeur de la frappe de recopie) que pour l'internaute questionneur (impossibilité de remonter à la source sans se déplacer à la bibliothèque, absence de rebond possible sur des informations complémentaires). Peu à peu donc, les répondants ont dans la mesure du possible privilégié des sources en ligne, qui présentent le double avantage de faciliter les copier-coller et de permettre à l'usager d'accéder immédiatement à la source voire d'aller plus loin très aisément. Ce parti pris, évidemment plus facile hors des domaines les plus patrimoniaux, est 
aujourd'hui généralisé, et l'équipe qui coordonne le Guichet estime que $60 \%$ des réponses ne font appel qu'à des sources Internet, $20 \%$ combinent des sources Internet en les appuyant sur des sources imprimées, et $20 \%$ sont établies à partir de seules sources imprimées.

Une telle évolution permet aux bibliothécaires qui ont l'occasion de rédiger des réponses d'apprendre à ajouter à leur maîtrise des contenus « matériels » une maîtrise de la recherche en ligne, et surtout d'apprendre à en combiner les avantages respectifs. Cette double compétence contribue à changer notablement le conseil aux visiteurs «physiques » qui se pressent à la bibliothèque, et intègre l'expertise acquise avec le service de $Q / R$ au service des visiteurs présentiels.

\section{DE LA RÉPONSE CONSTRUITE À LA PRODUCTION DE CONTENUS}

Deux ans après le lancement du GdS, en avril 2006, la BmL lançait un produit original, un magazine bibliographique en ligne proposant principalement des repères pour comprendre l'actualité, Points d'actu ${ }^{5}$. Des articles détaillés proposent de façon commentée et structurée des références de documents et de sites sur des sujets tels que « Les maladies rares sont-elles encore orphelines ? » ou "Vivre sa foi musulmane en Rhône-Alpes ».

La mise en œuvre de ce magazine, entièrement réalisé par les mêmes bibliothécaires qui rédigent des réponses sur Le GdS, était une façon de reformuler l'expertise de ces bibliothécaires. Jusque-là soumis à la pression forte mais imprévisible du flot des questionneurs du GdS, les professionnels pouvaient reprendre la main en élaborant cette fois-ci une offre délibérée de contenus. Points d'actu, malgré cette différence de positionnement, se situe bien dans la continuité du Guichet du Savoir :

- l'habileté acquise à rédiger des réponses souvent longues et argumentées a facilité le passage à la rédaction d'articles autonomes, parfois véritables dossiers de synthèse originaux ${ }^{6}$;

5. Points d'actu : < http://www.pointsdactu.org >

6. Voir par exemple ce dossier « La netcampagne des législatives 2007 en Rhône-Alpes : la course au Net et après » : < http://www.pointsdactu.org/article.php3?id_article=863 > (consulté le 3 mai 2010). 
- l'habitude prise avec les réponses du GdS facilite l'application d'une des exigences de Points d'actu : proposer parallèlement des références bibliographiques mais aussi et surtout des pistes fiables sur Internet, surtout, parce qu'un service proposé sur Internet doit toujours permettre de rebondir sur la Toile. Grâce à l'expérience acquise sur Le GdS, ce pari est plus facile à tenir sur Points d'actu !

- enfin, les recherches opérées pour répondre à des questions parfois récurrentes ou voisines sur Le GdS donnent des pistes et des armes pour construire un article dans Points d'actu. D'ailleurs, la maquette de Points d'actu propose pour chaque article une zone au sein de laquelle peuvent être rappelées certaines des réponses du GdS en rapport avec l'article. Parallèlement d'ailleurs, certaines réponses aux questions du GdS peuvent faire référence à une synthèse de Points d'actu!

\section{INTÉGRER LE CONTENU DES RÉPONSES DANS L'OFFRE DOCUMENTAIRE DE LA BIBLIOTHĖQUE}

Cette réutilisation croisée des contenus produits par les bibliothécaires, pour la BmL tantôt sur Le GdS tantôt sur Points d'actu, signale une autre originalité délibérée de ce dernier service de Q/R. La publication des questions et des réponses, leur conservation et aussi la duplication d'une partie de celles-ci dans une base thématique intégrée au forum génèrent à force d'accumulation une véritable offre documentaire autonome qui vient s'ajouter, au sein de la BmL, aux ressources bibliographiques, documents numérisés et articles de Points d'actu. La mutualisation des réponses apportées individuellement aux questionneurs est un argument fort en faveur d'une large ouverture du Guichet du Savoir à tous les types de demandeurs, quelle que soit leur origine géographique, dans la mesure où le service personnel dont ils ont pu bénéficier est réinjecté dans une offre documentaire dont 
bénéficient certes tous les internautes, mais au premier chef les Lyonnais. On a vu que la richesse informative de cette manne pouvait être utilisée sur d'autres services de façon volontariste, en sélectionnant par exemple quelques questions particulières du GdS pour Points d'actu. Par ailleurs, l'accessibilité permanente des questions et réponses publiées permet une forme de feuilletage au hasard, et $32 \%$ des visiteurs enquêtés en 2005 avouaient ne pas avoir posé de question mais se livrer régulièrement à ce butinage, en quelque sorte un quiz ou un apéritif culturel. Pour faciliter cette utilisation des réponses comme source d'information autonome (au sens de : mutualisée et dégagée du seul intérêt du questionneur initial), la webmestre a élaboré un fil RSS permettant de suivre non le flux des questions au fur et à mesure qu'elles sont posées - ce qui laisserait perplexe le lecteur -, mais le flux des questions au fur et à mesure qu'elles reçoivent une réponse : $8 \%$ des visites reçues en 2009 l'ont été par le biais de ce fil RSS.

\section{La mise en place de ce fil RSs a fait germer d'autres idées de dissémination}

Différents portails thématiques étant animés sur le site Web de la BmL $<$ http://www.bm-lyon.fr >, d'autres fils RSS ont été construits, permettant à titre d'exemple de suivre sur le portail Arts vivants < http://www.bmlyon.fr/artsvivants/ > les dernières réponses apportées par le département Arts vivants sur Le GdS.

Je disais plus haut que les quelque 30000 questions-réponses du GdS (chiffres au $1^{\text {er }}$ mars 2010) constituaient un gisement autonome d'information. Mais il fallait passer à l'étape suivante : la BmL dispose par ailleurs d'un catalogue de plus d'un million de notices bibliographiques, de bases de documents numérisés - essentiellement iconographiques et bientôt largement textuels -, de Points d'actu, de dizaines d'expositions virtuelles, des portails déjà signalés, analyses, etc. Chacun de ces types de ressources dispose de sa propre architecture d'organisation, de ses propres modalités de requêtes, de ses spécificités d'affichage. Pour un utilisateur lambda, naviguer entre ces multiples réservoirs est un parcours du combattant. Il fallait trouver le moyen d'organiser une interrogation multibases ergono- 
mique. Ce fut fait fin 2004 avec l'adoption d'un outil élaboré pour de très grosses entreprises ayant à gérer des millions de documents sous des formats hétérogènes, Autonomy ${ }^{7}$. Cet outil, fondé sur des analyses statistiques de formes, récupère dans un métacatalogue les données en provenance des diverses bases, les réorganise en fonction de poids statistiques, et permet une interrogation en langage naturel, les réponses apportées mêlant les diverses sources et les présentant selon un rang de pertinence calculé statistiquement. Cet outil fonctionne depuis fin 2004 à la BmL sous le nom de Catalog ${ }^{8}$.

Comme on peut le voir sur la copie d'écran ci-après, les résultats mêlent indifféremment notices bibliographiques, documents numérisés, articles de Points d'actu... et réponses du Guichet du Savoir.

\section{Catalog+, le métacatalogue multisources de la BmL (copie d'écran)}

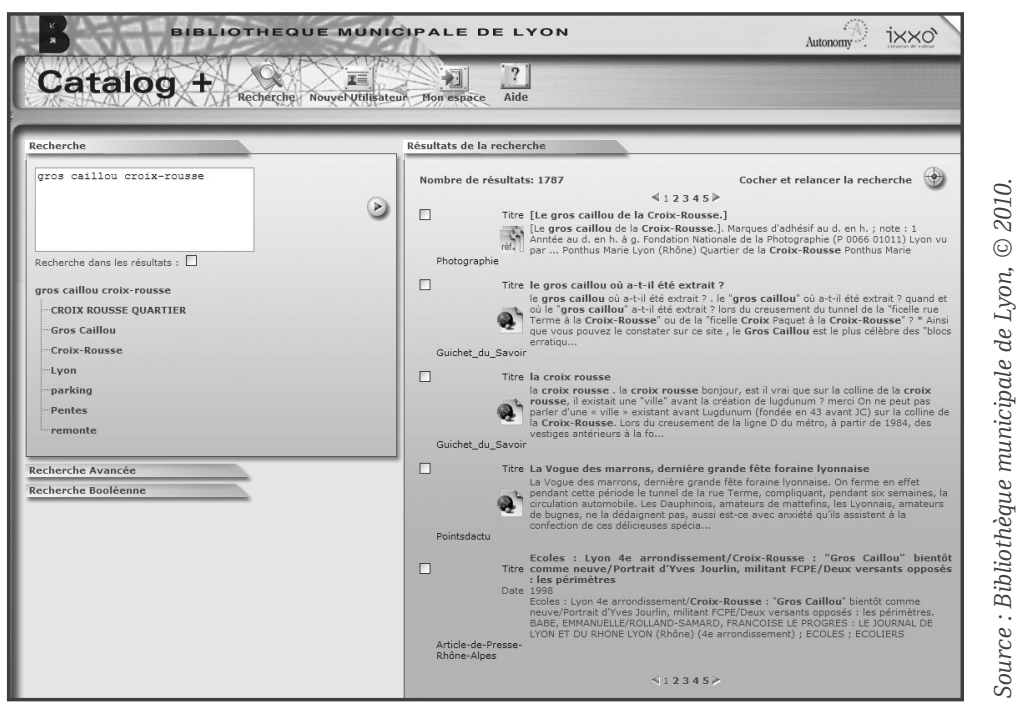

7. Parmi les solutions proposées par Autonomy, la BmL a adopté Retina < http://www.autonomy.com/ content/Products/idol-modules-retina/index.en.html > (consulté le 3 mai 2010).

8. < http://autonomy.bm-lyon.fr/retina/public/basic.do > (consulté le 3 mai 2010). 
En cliquant sur le titre d'une notice listée dans Catalog+, on arrive directement sur la page correspondante au sein de l'application originelle : par exemple, en cliquant sur une notice issue du GdS, on accède directement dans Le GdS à la question-réponse complète. La base de Catalog+ est actualisée quotidiennement pour tout ce qui concerne les contenus Web (hebdomadairement pour le catalogue bibliographique), ce qui garantit une bonne efficacité dans les résultats des requêtes. Ce parti pris de la BmL considère explicitement les réponses du GdS comme des produits documentaires à part entière : je n'en connais pas d'autres exemples.

En dépit de son nom, Catalog+ n'est pas fondamentalement un catalogue au sens traditionnel : il n'organise pas des notices normalisées, mais gère de l'information non structurée : il n'est efficace qu'en tant qu'il déstructure les notices du catalogue comme il aborde les réponses en langage naturel du Guichet du Savoir, pour les restructurer à sa façon en vue de pouvoir traiter ces différents contenus d'une manière uniforme. Ce processus montre de façon éclairante comment les réponses du GdS peuvent être intégrées de façon dynamique à l'offre documentaire globale de la BmL, ce qui serait évidemment impossible au sein d'un catalogue bibliographique de type MARC. Sans balayer les atouts spécifiques du SIGB, Catalog+ signale le passage de la bibliothèque gestionnaire de supports à la bibliothèque productrice et gestionnaire de contenus.

Il n'est pas certain que, hors pour certains échanges électroniques exigeant une confidentialité garantie, les services de $Q / R$ des multiples bibliothèques gagnent à séparer toujours nettement leur production de réponses construites des autres offres et services documentaires qu'elles proposent, si elles veulent non seulement intégrer et valoriser ce travail essentiel et souterrain, mais aussi enrichir leur offre globale. Si l'échange entre le bibliothécaire et son visiteur est éminemment personnel, la mutualisation de la production documentaire générée par cet échange me semble - sous réserve bien sûr pour le questionneur d'acceptation de ces modalités et d'anonymisation garantie - une exigence essentielle pour garantir le caractère public de nos établissements, l'enrichissement documentaire de leur offre, et l'intégration comme la valorisation documentaire du service de $Q / R$. 


\section{PARTIE II}

\section{RÉPONDRE : OUI, MAIS COMMENT?}

1. COMMENT RÉPONDRE ET QU'EST-CE QUE BIEN RÉPONDRE ? CONSTRUIRE LE SAVOIR-RÉPONDRE par Marie-Françoise Defosse

2. OFFRIR UNE BASE DE CONNAISSANCES : POURQUOI, POUR QUI, COMMENT?

par Marie Montano

3. LE CADRE JURIDIQUE DES QUESTIONS ET DES RÉPONSES

par Anna Svenbro

4. QUELS OUTILS POUR QUELS SERVICES ?

QUELLE TECHNOLOGIE CHOISIR ?

par Hélène Tardif

$+++++++++++++++++++++++++++++++++++++++++t$

5. COMMENT ÉVALUER UN SERVICE DE QUESTIONS-RÉPONSES EN LIGNE ?

par Nicolas Alarcon 


\section{1}

COMMENT RÉPONDRE ET par Marie-Françoise Defosse QU'EST-CE QUE BIEN RÉPONDRE ? CONSTRUIRE LE SAVOIR-RÉPONDRE

Si cette question, utilisée comme titre, est vaste et interpelle encore les professionnels des services de référence, l'objectif final réside bien dans la satisfaction des usagers. Et le recours de plus en plus fréquent des utilisateurs à ces services, leur succès incontestable en sont la preuve.

Sous-jacente à la question du "Comment répondre ", se profile celle de savoir ce qu'est une «bonne réponse », opposée à ce que serait une mauvaise réponse... Or, une réponse élaborée aujourd'hui pourra être différente demain, peut-être plus détaillée ou développée selon d'autres axes. Si l'existence des services de références en ligne n'est plus incongrue aujourd'hui, le « Comment répondre » que ce soit par e-mail ou par chat, se pose avec une actualité renouvelée.

Pourquoi se poser la question du « Comment répondre »? Pourquoi ne pas adapter ce qui se pratique dans les services de référence en présentiel ? En référence virtuelle, le médium utilisé pour répondre influence-t-il la manière de répondre ? Oui et non. Oui, parce que les écrits restent et les services de référence se sentent tenus à une plus grande rigueur en même temps qu'une plus grande recherche de qualité dans les réponses fournies. Non, parce que quel que soit le médium utilisé pour répondre, les savoirfaire convoqués lors d'une réponse sont toujours identiques : prendre en compte l'utilisateur et sa demande, utiliser une méthode de recherche d'information adaptée et formaliser la réponse. Nous ne pouvons que remarquer que ces questionnements, si vivaces lorsqu'il est question de services virtuels, ne se posent pas avec autant d'acuité, ni autant d'actualité pour le renseignement en face à face. 
Le répondant virtuel ne voit pas son usager, il ne peut pas l'accompagner dans les rayons de la bibliothèque, lui montrer l'utilisation du catalogue ou d'autres outils et il n'a pas de retour immédiat sur le travail de recherche qu'il accomplit... La référence virtuelle oblige à transposer, à dépasser ces impossibilités, à trouver d'autres voies. Le répondant doit mettre en œuvre des capacités de compréhension rapides et immédiates ; il doit faire preuve d'une curiosité permanente et d'une capacité à intégrer des nouveautés, des évolutions, le travail de ses collègues... Il doit accepter l'imprévu, la question elliptique, confuse ou mal formulée. Les demandeurs, de plus en plus nombreux ne sont pas toujours les cibles pressenties lors de la création du service. À titre d'exemple, un service de référence d'une université s'est vu proposer une offre de logement à louer à l'approche de la saison locative... Les services de référence sont accessibles sur Internet et les utilisateurs sont multiples, pas nécessairement étudiants, ni même de langue française... Le « Comment répondre » est à adapter sans cesse.

\section{ÉTABLIR DES CRITÈRES POUR CONSTRUIRE UN SAVOIR- RÉPONDRE}

\section{OBSERVER CE QUE FONT LES AUTRES}

Une manière de développer un savoir-répondre réside dans l'observation et l'analyse comparée de réponses accessibles en ligne, émanant d'autres services, et surtout de services différents ${ }^{1}$.

\section{ÉLABORER DES RĖGLES POUR LE « COMMENT RÉPONDRE » DE SON SERVICE}

Les règles $\mathrm{du}$ "Comment répondre » doivent être arrêtées, discutées, choisies, enrichies, modifiées et augmentées par l'équipe du service de référence. Si la réponse faite à un usager est individualisée, la construction d'un savoir-répondre doit être collective. De plus, le savoir-répondre doit évoluer dans le temps et faire l'objet d'évaluations fréquentes.

1. Notre analyse a porté sur les questions et les réponses des services suivants : BiblioSésame (Bpi), Ses@me (SCD Toulouse 2), Eurek@ (SCD Toulouse 3), Boomerang (BIU de Montpellier), le service Questions de la Cité de la Musique, Le Guichet du Savoir (BmL), Questions ? Réponses ! (enssib). 
Quatre critères essentiels sont à interroger :

\section{critère 1 : la forme visuelle de la réponse ou ce que verra le demandeur en lisant sa réponse.}

Ce critère implique d'analyser la structure même de la réponse, ses éléments permanents, récurrents ou non, ses codes, ce qui se voit...

La première impression visuelle donne le ton du service de référence. Et dans le ton, nous entendons bien sûr la stratégie de service. La réponse faite est courte ou longue, elle présente une structure, une articulation visibles ou pas, c'est-à-dire qu'à la lecture de plusieurs réponses faites par un même service, nous pouvons distinguer une façon de répondre. La réponse est très rédigée ou plutôt de style télégraphique, etc. Tous ces éléments sont le reflet des choix d'un service.

Quelles questions un service de référence doit-il alors se poser dans l'optique de développer son savoir-répondre ? Doit-il adopter une structure de réponse visible et identique pour toutes les réponses ? Doit-il formaliser une introduction, un développement, une conclusion de manière toujours semblable ? "Charter » sa mise en page, apposer le logo de son service ? Circonscrire la taille de sa réponse à 1 page, 2 pages... Dans ces domaines, l'offre des autres services montre des pratiques variées. Pas ou très peu de structure visible pour Le Guichet du Savoir, la Cité de la Musique ou la Bpi ; au contraire, une structure très visible pour le service de l'enssib ainsi que pour la plupart des services de références des bibliothèques universitaires.

Si les services oscillent entre une grande formalisation et peu ou pas de formalisation, la préconisation générale est d'homogénéiser les réponses. Si la formalisation n'est pas restituée dans la forme finalisée de la réponse, elle est cependant fortement conseillée pour penser la réponse à faire. Nous avons rencontré des services qui ne souhaitent pas rigidifier les pratiques des répondants en formatant les réponses. Le Guichet du Savoir ne formalise pas la structure de ses réponses mais en revanche, il utilise des codes typographiques, des couleurs, des mots soulignés, l'insertion d'images. Pour les services qui harmonisent, l'homogénéisation des réponses sert aux répondants - surtout aux novices, nouveaux entrants dans le service ; elle 
peut être aussi une étape nécessaire à la cohésion des équipes et aux enrichissements réciproques car le savoir-répondre d'un service est la résultante de l'amélioration du savoir-répondre de tous les répondants. De plus, pour les usagers, reconnaître la " patte » d'un service, savoir comment il présente les informations fournies, est un élément de confort. Et le confort fidélise. L'effet de visibilité est un facteur de notoriété à ne pas négliger à l'heure des réseaux et d'une possible concurrence entre les services.

\section{critère 2 : méthode de recherche, contextualisation de la réponse et ressources utilisées}

\section{Rendre visible la méthode de recherche ?}

Y'a-t-il des priorités, un ordre préférentiel pour présenter les ressources ? Comment les présenter ? Faut-il les accompagner de commentaires et d'explications ? Doit-on faire état de la méthode de recherche employée en préambule de la réponse, au cours de la réponse ? Est-il pertinent d'indiquer des mots-clés à l'usager ? D'aller jusqu'à fournir les requêtes possibles ? Ce sont des questions que tout service doit se poser. Pourquoi ? Parce que le service met en œuvre, utilise et répond selon une méthode de recherche documentaire. L'expertise du service repose sur cette méthode faite d'accompagnement à l'utilisation des ressources, de repérage de sources d'informations pertinentes et fiables, d'orientation vers des sources secondaires. Là encore, les attitudes sont variées : pas d'explicitation de méthode pour Le Guichet du Savoir, la Cité de la Musique, Questions ? Réponses ! de l'enssib... En revanche, un apport de méthode pour Ses@me, Eurek@, Boomerang et d'autres.

\section{Contextualiser la réponse ?}

Dans ce domaine, les services fonctionnent différemment. Certains reformulent la question et la contextualise en guise d'introduction (Questions ? Réponses !), d'autres non, tels Le Guichet du Savoir ou Bibliosésame. Le service Questions ? Réponses! indique ne plus fournir désormais de contextualisation dans ses réponses, - procédure en cours jusqu'alors -, afin de permettre une entrée plus directe et plus immédiate dans la réponse, pour réduire sa longueur. Toutefois, l'équipe des répondants continue à 
formuler une contextualisation qui n'est plus donnée au demandeur mais sert d'aide à la construction de la réponse, surtout si la question est dense.

\section{Quelles ressources utiliser?}

Faut-il utiliser en priorité les ressources de son établissement ? Démarrer par le catalogue ou des catalogues ? Prioriser les ressources électroniques ou ne rien indiquer en se contentant de fournir la réponse attendue?

Si Le Guichet du Savoir, Questions ? Réponses ! et BiblioSésame ne proposent pas, voire très peu, des ressources issues de leur établissement, en revanche, d'autres services le font. Ses@me propose toujours d'explorer en premier lieu le catalogue de l'université et le Sudoc, viennent ensuite les revues via les bases de données de l'établissement, enfin les ressources Internet.Eurek@ propose dans l'ordre, le catalogue de l'établissement, des revues, enfin des outils et des sources spécialisées tels Google Scholar, des bases de données disciplinaires (Pubmed, Francis, Pascal), des thèses... Le Guichet du Savoir et Questions ? Réponses ! répondent en trois temps, préférant proposer un découpage en niveaux d'informations plutôt qu'un découpage selon des types d'information : présentation de ressources fondamentales pour démarrer, puis dans un second temps de ressources annexes parfois très détaillées, enfin, dans un dernier temps de ressources pour aller plus loin. Il est à noter que les services virtuels anglo-saxons et canadiens préconisent l'utilisation de ressources électroniques en priorité.

\section{critère 3 : quelle rédaction pour la réponse ?}

Comment écrire ? Utiliser le « je », le « nous »... ? À quelle distance de l'utilisateur se placer? Préconiser, recommander, proposer des informations en liste ou faire des choix pour l'utilisateur? Si le chat peut permettre une proximité de vocabulaire, un style plus familier, des abréviations, en revanche l'écriture d'un e-mail varie d'un style littéraire et très écrit à un style plus télégraphique.

S'il est légitime de s'interroger sur le style de la réponse à rédiger, il faut également s'interroger sur l'intégration d'éléments invariants dans chaque réponse. Ces éléments sont la marque du service et montrent à l'utilisateur 
qu'un professionnel lui répond et l'accompagne et que la bibliothèque met en œuvre un service de réponses. On pourra donc réfléchir à la manière dont le service doit saluer son usager et l'inviter à poser sa question, à la manière dont le service peut conclure sa réponse, se présenter ou signer la réponse (en utilisant le nom du répondant ou le nom du service).

Ces éléments sont importants pour l'image du service de référence. Quelle que soit la réponse faite, ces trois éléments (formules d'introduction, de conclusion et signature) sont indispensables et doivent être récurrents. Les logiciels qui permettent de répondre par chat proposent des scripts automatisés pour ces éléments.

Enfin, le ton de la réponse est très important. Il importe de rester neutre, de ne pas être prescripteur auprès du demandeur, d'éviter les commandements, les «Il faut que... » et de ne pas porter de jugement, ni d'appréciation sur la question posée. Avant tout, le répondant manifestera de la bienveillance voire de l'empathie... En somme, il aura le sourire au bout des doigts en tapant sur son clavier !

\section{critère 4 : le marketing de la réponse}

Remercier l'utilisateur d'avoir utilisé le service de référence, le fidéliser en lui proposant de renouveler son utilisation du service, ne pas hésiter à l'envoyer vers d'autres services de la bibliothèque : Prêt Entre Bibliothèque, invitation à suivre des formations ou encore rendez-vous avec un bibliothécaire pour affiner sa recherche... sont des éléments qui participent du marketing du service. Un service de référence ne se pense pas seul ; le "Comment répondre » est une somme de services à valoriser.

\section{DES FONDAMENTAUX POUR LE SAVOIR-RÉPONDRE}

Quelles que soient les méthodes employées et les règles mises en place, les positionnements des services et les modes de réponses, des intangibles $\mathrm{du}$ « Comment répondre » se dégagent.

\section{s'arrêter sur la question posée}

La première étape du savoir-répondre consiste à s'arrêter sur la question posée et à recueillir tous les renseignements donnés par l'utilisateur : la 
manière de rédiger sa question, son vocabulaire et ses expressions, ses tournures de phrase, l'expression de son besoin d'informations, ce qu'il indique des recherches qu'il a déjà effectuées... Ce sont autant d'informations précieuses qui vont aider le répondant. Cette étape est valable pour les deux modes de réponse, que ce soit par e-mail ou par chat.

Les premières questions que le répondant doit se poser sont : que sais-je du demandeur et que m'apprend-il à travers sa question ? Que veut-il prioritairement et/ou accessoirement ? Et donc comment vais-je orienter ma réponse ? Vers quelles sources ? Avec quelles limites ? Cette étape, indispensable et obligatoire aide le répondant à situer le niveau de réponse attendu et à poser des limites à sa recherche.

En répondant par e-mail, le bibliothécaire ne peut mener l'entretien de référence. Le seul outil qu'il peut utiliser pour pallier ce manque est le formulaire $^{2}$. En chat, l'entretien de référence est possible... et fortement recommandé ! La situation virtuelle est idéale : un demandeur face à un répondant qui peut l'accompagner pas à pas.

Lorsque l'utilisateur questionne le service par e-mail, peut-on le recontacter pour lui faire préciser sa demande si elle est confuse ou semble incomplète ? Les équipes de répondants rencontrées disent non. Il est préférable de répondre à ce qui a été compris, quitte à ne pas aller trop loin et à inviter l'usager à repréciser sa demande, dans un second temps. Deux règles semblent donc en usage : ne pas recontacter l'utilisateur et toujours répondre, même si la demande est mal comprise.

\section{ÊTRE PRÉCIS ET CONCIS DANS LA RÉPONSE FOURNIE}

Dans ce domaine, le professionnel doit faire preuve de la plus grande pédagogie. Chaque mot compte, que ce soit dans un e-mail ou par chat. Cet effort de précision et de concision est aussi l'expression du professionnalisme et de l'expertise. Ce sont les raisons pour lesquelles les usagers s'adressent aux services de référence. La règle est donc de délivrer immédiatement la

2. Cf. l'article de J.-C. Houpier, « Service de questions-réponses et environnement de l'usager : quelle intégration ? », p. 59. 
précision et la concision attendues, sans attendre la phrase suivante pour donner ces renseignements. Il s'agit d'aller directement et complètement à l'information. Ce qui est vrai pour l'e-mail est encore plus parlant par chat car dans ce cas, immédiateté et réactivité priment.

\section{EXPLIQUER, COMMENTER ET ACCOMPAGNER À L'UTILISATION DES RESSOURCES?}

Là encore, plusieurs manières de procéder. La Cité de la Musique, BiblioSésame et Le Guichet du Savoir commentent peu, voire pas du tout, et délivrent une réponse sans explications. Ce dernier a choisi de donner une réponse précise et pas une orientation. Et nul ne peut mettre en doute le succès du Guichet du Savoir. L'éducation à l'information se fait ainsi à travers la somme des réponses, la fidélisation des usagers, la recherche possible dans les réponses en ligne. Dans les bibliothèques universitaires, la situation est différente. Peu ou prou, par e-mail ou par chat, les répondants commentent et expliquent.

Les répondants de BiblioSésame proposent à l'usager de chercher dans le catalogue de la bibliothèque, suggèrent des mots-clés et attirent l'attention de l'usager sur l'orthographe de ces derniers, expliquent l'utilisation des opérateurs de requête dans les bases de données et sur Internet, commentent leurs choix.Eurek@procède de la même manière et explique la structure des bases de données ; de plus, il rappelle l'existence d'une aide à la recherche en ligne disponible sur le site Web de l'établissement. Boomerang aide au choix des outils à consulter et des mots-clés à utiliser. Conçu initialement pour être principalement une aide méthodologique à la recherche d'informations, le service semble désormais mixer sa première approche avec celle qui consiste à fournir des ressources. Rue des Facs indique dans sa charte que le service développera aussi dans sa réponse une aide méthodologique.

Si les professionnels sont partagés quant à la manière de procéder, accompagnement à la recherche ou fourniture d'informations, il n'en demeure pas moins que les explications concernant les outils, les commentaires de ressources et l'accompagnement dans la démarche soulignent aussi l'ex- 
pertise des bibliothécaires, tout comme ils fidélisent les utilisateurs... Tout du moins, ceux qui souhaitent acquérir une méthode de recherche! $\mathrm{Au}$ sein des services de référence, la question du « Comment répondre » à l'utilisateur fait débat et tous les répondants ne s'accordent pas entre eux sur la manière de faire : délivrer une réponse ou accompagner la recherche $^{3}$ ? Ne pourrait-on imaginer une combinaison des deux?

\section{CONSTRUIRE DES OUTILS POUR MIEUX RÉPONDRE ?}

Le «Comment répondre », le savoir-répondre et le bien répondre sont les résultats d'un travail de l'équipe des répondants du service. La création et l'utilisation d'outils s'avèrent ainsi nécessaires ; ils servent autant à élaborer le savoir-répondre qu'à aider chaque répondant placé en situation de réponse.

\section{POUR MIEUX CONNAÎTRE LE DEMANDEUR}

En situation de référence en face à face, cette connaissance améliorée passe par l'entretien de référence. Cet entretien étant impossible à réaliser par e-mail, le formulaire accompagnant la question et l'étape de décryptage de la réponse sont des outils importants. Le chat, quant à lui, permet de mener l'entretien de référence.

\section{POUR FIXER LES LIMITES DES RÉPONSES POSSIBLES}

Les chartes des services permettent de délimiter par avance ce à quoi le service répondra, ce à quoi il ne répondra pas. Ici encore, les objectifs diffèrent d'un service à l'autre .

Si les chartes évoquent le cas des questions hors sujet, la réalité des fonctionnements est différente et ne suit pas toujours les chartes à la lettre. Dans la mesure où les services de référence sont accessibles par Internet, de plus en plus de questions y sont posées. Les frontières ont tendance à exploser et le cas des questions qui se situent hors du périmètre de réponse

3. Cf. l'article de C. Di Pietro, « Construire un service de référence généraliste : l'exemple du Guichet du Savoir de la Bibliothèque municipale de Lyon », p. 152.

4. Cf. l'article de C. Nguyen, « Typologie des services de questions-réponses », p. 146. 
du service, se pose fréquemment. Pour tous les services avec lesquels nous nous sommes entretenus, la règle est de toujours fournir une réponse. Si la demande n'est pas dans le périmètre accepté, il pourra s'agir d'une réorientation vers un autre service de référence ou de la fourniture d'une première piste très générale.

\section{HOMOGÉNÉISER LE SAVOIR-RÉPONDRE À TRAVERS DES GUIDES DE RÉPONSE}

Nous ne trouverons pas de guides du savoir-répondre en ligne. Ces outils sont rarement formalisés par les services de référence et, lorsqu'ils le sont, leur utilisation relève d'un usage restreint aux membres de l'équipe des répondants. De plus, ils oscillent entre plusieurs voies : organiser et formaliser ou pas, pas encore, pas maintenant... Une rapide enquête menée auprès de quelques services a révélé que certains d'entre eux ne voyaient pas l'utilité de construire de tels outils. Les bibliothécaires savent chercher et donc savent répondre !

Le service Questions ? Réponses ! a formalisé un guide du « Savoir-répondre et de la rédaction d'une réponse ". L'objectif est posé dès le préambule : il ne s'agit pas de construire des réponses types mais de viser à une harmonisation des pratiques. Le guide traite des points suivants : la prise en compte de la situation et du profil du demandeur, l'affichage de règles d'objectivité et de bienveillance dans la réponse, la fourniture de ressources les plus complètes possibles avec une hiérarchisation allant des sources primaires aux sources secondaires, le commentaire des ressources qui est une plus-value du service, la relecture indispensable de la réponse. Le guide présente la règle des trois tiers : $1 / 3$ du temps pour analyser la question, $1 / 3$ pour la recherche effective et $1 / 3$ pour la rédaction et la mise en forme de la réponse.

Ubib.fr dispose, quant à lui, de deux guides distincts. Un mémento du savoirrépondre par e-mail et un mémento du savoir-répondre par chat. Si le premier a été construit lors du démarrage du service, le second a été formalisé lors d'un coaching - formation des équipes de répondants par chat. Chaque séance de formation a permis à l'ensemble des stagiaires d'enri- 
chir ce guide. La construction de cet outil a permis de mettre en exergue les points suivants : quel est le cadre de déroulement d'une session de chat et ses principales étapes, quelles sont les règles d'écriture à adopter en chat, les règles de ton à utiliser dans l'écriture, comment répondre dans des cas spécifiques identifiés par les répondants, quels outils et services d'informations sont à construire en complément pour que les répondants puissent mener à bien leurs sessions.

Le « Comment répondre » ou " comment bien répondre » se construit au sein d'un service de référence avec les répondants. Poser des règles pour le " savoir-répondre " aide à organiser les contenus d'une réponse, apporte une valeur ajoutée certaine, permet d'harmoniser un travail souvent collectif et donne toute sa spécificité au service de référence. Il n'y a pas de modèle de bonne réponse car "plus on répond, mieux on répond » (Catherine Jackson).

\section{comment établir le guide du savoir-Répondre de son service?}

Outil d'aide auquel chacun se reportera, il se doit d'être exhaustif et précis car il encadre la pratique commune.

Des ateliers de travail où l'équipe des répondants examine quelques questions/réponses du service serviront à repérer des bonnes et des mauvaises pratiques, les situations dans lesquelles l'équipe doit fonctionner sur le même modèle.

Ensuite, un second temps sera consacré à la mise en forme et à la rédaction du document sur la base des résultats des ateliers.

Enfin, une fois ou deux par an, la révision de ce guide en équipe, permettra de réactualiser les pratiques et de faire le point sur son usage. 


\section{2}

OFFRIR UNE BASE DE CONNAISSANCES : POURQUOI, POUR QUI, COMMENT?

Cet article est rédigé à partir de mon expérience à la Bpi, au service BiblioSés@me. Créer un réservoir de qualité à partir de questions qui ont été posées dans le cadre d'un service questions-réponses en ligne en proposant les réponses données est une tâche chronophage et ardue qu'il faut bien différencier de la gestion d'une FAQ qui affiche des questions récurrentes souvent d'ordre pratique, généralement évacuées des bases de connaissances documentaires.

\section{L'intérêt d'une base de connaissances (BC) est triple :}

- donner à l'usager une meilleure visibilité du service de Q/R en ligne, voire une autonomie de recherche ;

- valoriser le service en affichant une expérience et une expertise ;

- permettre aux professionnels de capitaliser leur travail pour des recherches ultérieures, pour modéliser la méthodologie de la recherche et la présentation des résultats. La base sert aussi de réservoir aux bibliothécaires qui peuvent reprendre tout ou partie de questions déjà traitées car l'internaute attend souvent une réponse «personnalisée ».

Avant de se lancer dans un tel projet, il faut toujours penser à la récupération éventuelle des données en cas de changement de logiciel, ce qui induit de se poser la question de leur propriété. Sans compter que pour avoir le droit de publier les questions il est indispensable d'obtenir l'autorisation de celui qui les a posées, même si toute trace d'identification est effacée avant mise en ligne. Ce pourquoi les formulaires demandent d'emblée l'autorisation de publier à l'internaute. Il est également indispensable de proposer un certain volume de questions et de réponses pour assurer la crédibilité de l'offre.

Toutes les questions posées ne sont pas "récupérables » : un tri rigoureux est nécessaire et il faut dans un premier temps établir des critères de sélec- 
tion. Ce qui signifie qu'un administrateur de base est indispensable. Il est préférable que ce dernier participe à l'activité courante pour bien en mesurer les limites, la portée, les tenants et les aboutissants.

Le traitement automatique n'est donc pas envisageable pour un résultat de qualité. La récurrence n'est pas un critère suffisant, même s'il peut être retenu pour des thèmes d'actualité ponctuelle. Certains logiciels permettent d'introduire des questions artificiellement afin par exemple de proposer des fiches méthodologiques, comme des guides de recherche (exemple : Comment trouver des études de marché ?). À la différence d'une interrogation à la volée sur Google, la richesse vient de la rareté d'une question qui a nécessité de longues recherches et un incontestable savoir faire du bibliothécaire.

Les professionnels en charge de ce travail sont donc tenus de balayer avec une régularité de métronome les questions qui ont été posées, en évaluer la pertinence par rapport à l'alimentation de la base de connaissances et de faire des choix. Lorsque celles-ci sont retenues, ils doivent les corriger (fautes d'orthographe, de syntaxe, de français, etc.), les anonymiser, voire compléter la recherche documentaire elle-même (certains supports ou sources d'information ont pu être oublié dans le stress de l'obligation du respect du délai de réponse).

Vient ensuite la question de l'architecture de la base telle qu'elle est proposée à l'interrogation pour l'usager et de l'indexation de chaque question. Pour des champs délimités de la connaissance, autrement dit pour des domaines experts, comme le droit, la médecine, la bibliothéconomie, etc. qui peuvent aisément s'appuyer sur un thesaurus ou sur une liste établie de concepts spécifiques, auquel le public idoine est habitué, la tâche est relativement aisée, en indexation comme en structure d'offre d'interrogation, ces dernières s'appuyant sur des outils préexistants ou construits spécialement pour cet objectif particulier.

Il en est tout autrement pour les services dits « grand public ». Aujourd'hui, la question cruciale est : comment ce public, habitué sans compétences particulières à l'interrogation avec des outils de type Google, procède-t-il ? Indexer une question-réponse avec cette problématique en tête est de nos jours au cœur de notre métier quand l'objectif est de servir l'internaute et son autonomie. S'approcher, sans outils, de ce qu'on appelle le langage 
naturel est un défi pour les professionnels. Cependant, cette situation précisément donne sens à notre métier et aux services de Q/R ; pour l'heure, qui sait mieux qu'un bibliothécaire ou un documentaliste jongler avec les listes d'autorités, les thesauri, les opérateurs booléens, les bases de données, les structures de sites Web libres d'accès?

\section{TABLEAU COMPARATIF DES MODES D'INTERROGATION DES BC}

\begin{tabular}{|c|c|c|c|c|}
\hline & Bibliosésame & $\begin{array}{l}\text { Questions ? } \\
\text { Réponses ! } \\
\text { (enssib) }\end{array}$ & $\begin{array}{l}\text { Le cuichet } \\
\text { du savoir }\end{array}$ & $\begin{array}{l}\text { Questions-santé (cité } \\
\text { de la santé, cité des } \\
\text { sciences) }\end{array}$ \\
\hline : & $\begin{array}{l}\text { Interrogation } \\
\text { «plein texte ». }\end{array}$ & $\begin{array}{l}\text { Structuration par } \\
\text { domaines et par } \\
\text { mots clés } \\
\text { Idem }\end{array}$ & Feuilletage & $\begin{array}{l}\text { Affichage des dernières } \\
\text { questions posées et } \\
\text { possibilité d'interroger } \\
\text { l'ensemble de la base. }\end{array}$ \\
\hline 造 & $\begin{array}{l}\text { Regroupement par sujets. } \\
\text { Intérêt des usagers pour } \\
\text { un thème précis, qui trou- } \\
\text { veront par ce biais des } \\
\text { informations connexes. }\end{array}$ & $\begin{array}{l}\text { Question relative- } \\
\text { ment ciblée, } \\
\text { généralement } \\
\text { opérationnelle sur } \\
\text { le noms propres ou } \\
\text { les termes } \\
\text { spécifiques. }\end{array}$ & $\begin{array}{l}\text { Oriente } \\
\text { l'usager } \\
\text { sur le } \\
\text { service et } \\
\text { peut } \\
\text { l'inciter à } \\
\text { s'en servir. }\end{array}$ & $\begin{array}{l}\text { Reflète les préoccupations } \\
\text { du moment des } \\
\text { internautes et donne ainsi } \\
\text { un reflet de l'actualité des } \\
\text { questions du domaine } \\
\text { spécifique. }\end{array}$ \\
\hline 苞 & $\begin{array}{l}\text { Peut, comme avec la } \\
\text { plupart des méta-moteurs, } \\
\text { générer beaucoup de } \\
\text { «bruit », surtout si le } \\
\text { balayage est fait sur le } \\
\text { texte de la question et de } \\
\text { la réponse. Idéalement, } \\
\text { limiter le balayage du } \\
\text { moteur de recherche au } \\
\text { champ de la question } \\
\text { et/ou au champ de } \\
\text { l'indexation. }\end{array}$ & $\begin{array}{l}\text { Des questions } \\
\text { relatives au } \\
\text { domaine peuvent } \\
\text { être dans d'autres } \\
\text { champs échappant } \\
\text { à l'interrogation. }\end{array}$ & $\begin{array}{l}\text { Peut } \\
\text { donner une } \\
\text { image } \\
\text { partielle de } \\
\text { l'offre de } \\
\text { service. }\end{array}$ & $\begin{array}{l}\text { L'absence d'indexation en } \\
\text { amont limite les } \\
\text { possibilités d'interrogation } \\
\text { «scientifique » par } \\
\text { exemple avec gestion de } \\
\text { termes équivalents. }\end{array}$ \\
\hline 总 & Question Point & QuestionPoint & $\begin{array}{l}\text { Invision } \\
\text { Power Board }\end{array}$ & Content server \\
\hline
\end{tabular}

Dans tous les cas, la promotion de ces services est essentielle aux vues des énergies et compétences qu'ils mobilisent, à juste titre, car l'avenir est semble-t-il résolument aux services de proximité, paradoxalement à distance. 


\section{3}

LE CADRE JURIDIQUE par Anna Svenbro

DES QUESTIONS ET

DES RÉPONSES

\section{LA CONFIDENTIALITÉ}

Le traitement automatisé des données personnelles est généralisé et leur utilisation malveillante possible. La confidentialité dans le cadre des services de questions-réponses est donc cruciale. La loi du 6 janvier 1978 relative à l'informatique, aux fichiers et aux libertés, modifiée par la loi du 6 août 2004, s'applique à ces services. L'usager dispose des droits d'information, d'opposition, de rectification, de copie et d'accès aux données le concernant. Un fichier constitué dans le cadre d'un service de Q/R ne peut pas alimenter sans son autorisation les listes de diffusion de la bibliothèque, il faut garantir l'anonymisation (automatisée dans certains logiciels) des données en cas d'exploitation ultérieure (formations internes, évaluation du service), sous peine de saisie de la CNIL. Les problèmes de confidentialité peuvent aussi affecter les contenus, dans le cas par exemple des références liées à des travaux devant rester confidentiels jusqu'à publication. L'information de l'usager est essentielle, et l'attitude adoptée quant à la collecte des données et contenus, alors qu'il faut garantir une réponse adéquate et personnalisée, relève d'un compromis entre principe de précaution, respect du droit et service à l'usager.

\section{L'EXERCICE ILLÉGAL DES PROFESSIONS RÉGLEMENTÉES}

Le bibliothécaire est sollicité dans le cadre des services de Q/R pour son expertise quant à fournir l'information. Or, l'usager risque de le solliciter comme s'il était expert quant à cette information, risque d'autant plus grand si le service est spécialisé. Le domaine médical est exemplaire. L'article L.4161-1, $1^{\circ}$ du Code de la santé publique est formel, le bibliothécaire doit se montrer vigilant de ne pas tomber sous le coup d'une accusation d'exercice illégal de la médecine, s'agissant de questions impliquant même 
l'amorce d'un diagnostic ou d'une prescription médicale. Le domaine du droit n'échappe pas non plus au problème. Le bibliothécaire ne doit pas ébaucher de conseil juridique (loi n ${ }^{\circ}$ 1-1130 du 31 décembre 1971, art. 54 modifié par la loi $n^{\circ}$ 97-308 du 7 avril 1997 notamment, puis loi $n^{\circ} 90$ 1259 du 31 décembre 1990). L'exercice illégal de la médecine et celui du droit sont des délits passibles de sanctions pénales, civiles, et disciplinaires, dans le cas de la fonction publique d'État selon ce qui est prévu par les articles 66 et 67 de la loi $n^{\circ} 84-16$ du 11 janvier 1984, modifiée par la loi $n^{\circ}$ 91715 du 26 juillet 1991 (Chapitre VIII : Discipline).

\section{LE DROIT D'AUTEUR}

À l'heure des lois DADVSI du $1^{\text {er }}$ août 2006, Création et Internet du 12 juin 2009 et de la préparation de l'Anti-Couterfeiting Trade Agreement (ACTA), le rapport des services de Q/R au droit d'auteur est d'une brûlante actualité : ils y sont soumis comme les autres services de la bibliothèque. La tâche est ardue, avec la multiplication des contrats et licences d'accès aux ressources. Les services de $Q / R$ utilisent toutes les ressources de la bibliothèque (références bibliographiques, citations, etc.). Certaines sont présentes sous forme numérique. Mais font-elles partie des fonds de la bibliothèque ou sont-elles concédées via une licence par le prestataire de contenus en ligne ? Un service de Q/R ne peut faire d'entorse au contrat. Respecter le Code de la propriété intellectuelle, les limites de l'exception de courte citation introduite par son art. L.122-5 $3^{\circ}$ a., dont la brièveté sera laissée à « l'appréciation raisonnable » du rédacteur ${ }^{1}$, utiliser de préférence des contenus sous licence Creative Commons, se soucier des conditions d'utilisation des ressources de la bibliothèque est donc impératif dans le cadre d'un service de Q/R.

1. Cependant, on peut fournir du texte intégral à un usager affilié à l'institution couvert par la licence d'utilisation. 


\section{4}

QUELS OUTILS POUR QUELS par Hélène Tardif SERVICES ? QUELLE TECHNOLOGIE CHOISIR?

Un service de Q/R en ligne ne va pas sans une technologie pour l'appuyer. De l'utilisation des systèmes de communication déjà en place à l'achat d'un logiciel spécialisé pour les bibliothèques, toute une gamme d'outils commerciaux ou en libre accès est disponible. Faire un choix éclairé nécessite une réflexion qui dépasse l'aspect purement technologique. En effet, le type d'outil choisi aura une incidence sur le service offert, sur le personnel et sur la récolte des statistiques. Le choix du logiciel ne devrait donc pas être la première étape du processus de décision. Ces derniers changent constamment et au moment de faire la sélection d'un outil il faut déjà avoir franchi quelques étapes et avoir une idée générale de la vocation du nouveau service.

\section{AVANT DE CHOISIR}

Une période de questionnement précède donc l'étape du choix ${ }^{1}$. Quels besoins désire-t-on combler ? Étendre les heures d'ouverture du service de référence ou répondre au changement de pratique documentaire des usagers ? Qui espère-t-on voir utiliser le service ? Les usagers ou la communauté environnante, les jeunes ou les adultes. Quels types de questions sont susceptibles d'être posées ? Quels bénéfices en tireront les usagers et le personnel de la bibliothèque ? Quelles sont les contraintes à respecter? Pour aider les responsables de projet à faire un choix éclairé, Kathleen Kern, propose une grille d'évaluation qui confronte les caractéristiques des logiciels avec les priorités de l'institution (cf. tableau 1). La feuille de travail à élaborer demande de bien réfléchir aux besoins de la bibliothèque car il faudra ensuite donner une valeur aux caractéristiques des logiciels candi-

1. Cf. l'article de J.-P. Accart, « La démarche projet en vue de la création d’un service de questions-réponses en ligne », p. 16. 
dats. La période de réflexion préliminaire aura déjà permis de se faire une idée du type de service et de la catégorie d'outils à examiner. Cet exercice de comparaison et de classement doit être une des dernières étapes de tout le processus, considérant que l'outil comme tel ne doit pas influencer les autres décisions.

\section{DESCRIPTION DES PRINCIPAUX OUTILS}

\section{L'ÉVOLUTION DES OUTILS UTILISÉS POUR FAIRE DE LA RÉFÉRENCE EN LIGNE}

Le courriel a été utilisé pour communiquer avec les usagers dès que les bibliothèques ont affirmé leur présence sur Internet ; leur intégration dans l'organisation des services s'est faite naturellement. En revanche, l'histoire des logiciels de référence en ligne est plus mouvementée. Au début des années 2000, plusieurs bibliothèques universitaires (surtout américaines) commencent à tester des logiciels commerciaux de type centre d'appel sur le Web pour répondre aux besoins des usagers distants. Devant ce marché potentiel, plusieurs compagnies décident de lancer des logiciels adaptés à la réalité des bibliothèques. Ces outils, qui permettent de clavarder (chat), de conaviguer ou de prendre le contrôle à distance de l'écran de l'usager, font une entrée remarquée un peu partout dans le monde. Vers 2005, on observe un essoufflement du côté des logiciels commerciaux de référence en ligne. En effet, de nombreux ratages techniques, une certaine lourdeur d'utilisation, des problèmes de compatibilité leur sont reprochés. Pendant ce temps, la messagerie instantanée (MI), offerte en complément ou en remplacement des logiciels plus complexes, impose sa présence. Nouveaux dans les bibliothèques, les services commerciaux de MI étaient déjà bien connus des internautes et très populaires. Aujourd'hui, il n'est pas rare de voir une bibliothèque mettre à la disposition de ses usagers deux ou trois services de Q/R fonctionnant en parallèle. Quant au SMS, certaines bibliothèques l'ont implanté dès 2004, mais plusieurs observateurs du milieu considèrent que c'est en 2009 que les services de "référence mobile » ont réellement pris leur essor. Désormais, SMS, blog, Facebook font partie du vocabulaire de la prestation des services de référence. Et c'est sans compter les nouveaux logiciels ou gadgets (vidéos à la volée, signets, voix 
sur $\mathrm{IP}^{*}$ ) visant à faire de la session de clavardage une expérience multimédia.

\section{LES OUTILS DISPONIBLES}

Pour communiquer avec les usagers par l'entremise d'Internet les bibliothèques d'aujourd'hui ont l'embarras du choix. Pour simplifier le portrait d'ensemble des outils disponibles, il suffira de retenir que les services de Q/R se divisent en deux grands groupes.

Les services asynchrones où la communication s'effectue en différé (il y a un délai de durée variable entre l'envoi du message textuel et la réception de la réponse).

Les services synchrones où la rapidité de transfert des messages donne une impression d'interactivité, de conversation en temps réel. Les choses se complexifient rapidement selon les organisations et les niveaux de services. Une typologie détaillée des services de Q/R en ligne est présentée dans la partie IV, p. 145.

\section{COMMUNICATION ASYNCHRONE courriel}

Il s'agit de l'outil de Q/R le plus ancien, utilisé dans la grande majorité des bibliothèques. Toutes les institutions se servent déjà du courrier électronique pour les communications internes et la plupart des usagers possèdent un compte chez un fournisseur Internet ou utilisent un service courrier Web gratuit. Dans sa forme la plus simple, le service de Q/R se présente comme un lien hypertexte (adresse de courriel cliquable) placé sur la page d'accueil du site Web de la bibliothèque. L'adresse de courriel mène vers un compte de courrier électronique (compte dédié partagé par des membres du personnel ou arrivant sur toutes les boîtes personnelles). «Afin que l'utilisateur repère immédiatement le service, celui-ci doit porter un nom explicite, du type : Posez votre question ou Contactez un bibliothécaire » [Accart, $2008]^{2}$. L'usager pose sa question et s'attend à recevoir une réponse selon 
le délai annoncé. En général, une page du site explique les modalités du service. Le courriel possède l'avantage de pouvoir être conservé pour consultation ultérieure. Cet atout peut devenir un inconvénient sans un système de gestion des messages efficace (qui fait le suivi, qui répond, comment se fait l'archivage).

\section{Formulaire web}

Service similaire au courriel sauf que l'usager est dirigé vers un formulaire Web à remplir. Cette façon de faire est probablement la plus répandue. Techniquement, la page Web contient un script qui permet d'envoyer les données saisies à une boîte de courrier électronique (BAL). Le script est un bout de code HTML inséré dans une page existante, ce que tout administrateur de système ou programmeur Web peut facilement fournir. Un formulaire typique comporte un bloc de champs obligatoires où l'usager s'identifie (le formulaire peut être maillé avec le protocole d'authentification de l'institution) et un bloc où il doit préciser l'objet de sa requête ${ }^{3}$. Une bibliothèque qui ne souhaiterait pas installer elle-même un service de référence asynchrone peut se tourner vers un logiciel commercial de $Q / R$ qui propose un module de gestion du courrier électronique.

\section{SMS}

La messagerie texte (Short message service, SMS, Texto) est un système utilisé pour envoyer et recevoir des messages vers un téléphone cellulaire (ou autre appareil de poche) ou à partir de celui-ci. Devant l'omniprésence de la téléphonie mobile et l'usage quotidien du SMS par les jeunes, de plus en plus de bibliothèques mettent en place des services de "référence mobile ». L'interopérabilité de ce système rend possible différentes options de services. La plus simple : un numéro de téléphone mobile réservé au service de référence et un appareil utilisé par le personnel. Ce modèle requiert peu d'investissement mais il impose au personnel de devoir taper du texte sur un minuscule clavier. Une autre option est celle où le SMS de l’usager est transféré dans un logiciel de MI opéré par le personnel. L’usager

3. Cf. l'article de J.-C. Houpier, «Service de questions-réponses et environnement de l'usager : quelle intégration? ", p. 59. 
doit utiliser un numéro spécial et le coder dans son appareil, ce qui peut être une barrière. Pour la bibliothèque une complication possible est l'obligation de gérer un autre point de service. La dernière option est un service de passerelle SMS qui transforme le message texte envoyé par l'usager en un courriel, lequel est transféré dans la BAL du service de référence. Le courriel de réponse est à son tour transformé en SMS et transmis à l'usager. Ici, l'inconvénient est que beaucoup de bibliothèques n'ont pas le niveau d'expertise requis pour mettre en place une telle technologie [Stahr, 2009]. Les institutions intéressées pourront recourir aux services de compagnies qui commercialisent des systèmes de référence par SMS.

Par exemple : SMSreference (Altarama), utilisé par la Southeastern Louisiana University Library

< http://www.selu.edu/library/askref/index.html > et Text-a-Librarian (Mosio), utilisé par Yale University Library

< http://www.library.yale.edu/reference/asklive/index.html >.

Le protocole SMS autorise 160 caractères maximum espaces inclus. Cette limite de taille est un élément important à considérer à l'étape de la définition du mandat du service. Ce carcan a peu d'impact sur les questions à réponse rapide comme les renseignements sur la bibliothèque ou celles ayant trait aux transactions effectuées par l'usager, en revanche les questions nécessitant un entretien de référence s'accommodent mal de ce support. La faisabilité de répondre à des questions de référence en utilisant de courts messages a été souvent questionnée. On se demande si la petite taille de la communication textuelle réduit l'efficacité de la référence par SMS. Les expériences sont encore limitées mais les bibliothèques ayant adopté cet outil rapportent un bon taux de satisfaction. Une stratégie adoptée étant de répondre de manière générique et de diriger l'usager voulant aller plus loin vers un autre service (courriel, téléphone) [Stahr, 2009].

\section{Blog}

Il est très simple de créer un blog et de l'utiliser comme vitrine pour sa bibliothèque. Présenté sur la page d'accueil du site Web, il pourra servir à 
publier des annonces à la communauté. Autrement, il est possible d'en faire un service qui ressemble à une FAQ en y affichant des questions et réponses reçues de différents canaux. Les commentaires des lecteurs peuvent venir alimenter la base de Q/R. Le blog est un outil simple à implanter (ouvrir un compte sur une plateforme de publication de blogues comme Wordpress, Overblog ou Blogger, prévoir un système de gestion de contenu) et représente un investissement minimal ${ }^{4}$.

\section{Forum}

Le forum Internet est plus ancien que le blog mais résiste à l'épreuve du temps. Il s'agit d'une application Web qui génère un espace de discussion publique (ou réservé à une communauté). Les questions et réponses sont archivées et tous les usagers peuvent bénéficier de la même information. Contrairement au blog aucun commentaire ne peut être ajouté par les usagers. Au fil du temps une base de connaissances se construit avec les données validées par les modérateurs du forum. Le Guichet du Savoir de la Bibliothèque municipale de Lyon en est un bel exemple d'utilisation.

\section{COMMUNICATION SYNCHRONE}

Les outils permettant de démarrer un service de référence en temps réel sont variés et, paradoxalement, les emprunts technologiques mutuels font que les différences tendent à s'estomper. La terminologie anglaise porte aussi à confusion.

\section{Logiciel de type centre d'appel sur le web ou plateforme web}

Dans la littérature anglo-saxonne, on utilise les termes Web contact center software, Vendor-based chat, Chat software, Virtual reference software pour désigner les logiciels commerciaux par opposition à ceux basés sur la messagerie instantanée. Les compagnies les plus actives auprès des bibliothèques font la promotion de ce type d'outil en parlant de " service de gestion de la référence ». Il s'agit de logiciels évolués qui intègrent des modules correspondants à différents services : le clavardage, le courriel, une passerelle

4. Par exemple : Ask the ALA Librarian < http://americanlibrariesmagazine.org/askthelibrarian > (consulté le 6 mai 2010). 
SMS. Les compagnies ont investi beaucoup d'énergie dans la fonction de clavardage mais, rapidement les bibliothécaires ont constaté qu'il fallait se concentrer sur la gestion des appels pour assurer une prise en charge efficace de chaque usager. En effet, il est important de pouvoir accepter des appels multiples, de permettre à plusieurs opérateurs de travailler en simultané, de gérer la file d'attente (s'assurer qu'un appel est remis en file d'attente si le service est occupé) et d'obtenir de l'information sur l'appel entrant (adresse IP, navigateur). Des fonctions comme la conavigation (mode avancé où le bibliothécaire et l'usager peuvent contrôler la navigation sur les pages Web, tout mouvement de l'un étant visualisé par l'autre), le partage de fichiers (l'envoi de fichiers externes par le biais du logiciel), la possibilité de pousser des captures d'écran à l'usager (le push) sont intéressantes, mais sous-utilisées selon l'expérience vécue par de nombreuses bibliothèques. Les facteurs déterminants sont plutôt la rapidité et l'efficacité de la communication, d'où l'utilité des messages préenregistrés et automatisés. Deux autres caractéristiques des logiciels de type centre d'appel sont notables : leur module d'administration raffiné (collecte de statistiques, production de rapports, gestion des comptes) et la création d'une base de connaissances à partir des questions posées. La popularité de la MI et du Web 2.0 n’a pas manqué d'influencer les compagnies, ainsi la simplification (étapes à franchir avant de dialoguer avec un bibliothécaire), les gadgets logiciels et les passerelles vers le SMS font maintenant partie de l'offre marchande. Malgré tous leurs attraits, ces logiciels restent coûteux et demandent un plus grand engagement du personnel, c'est pourquoi ils sont plus implantés dans les bibliothèques et les regroupements qui reçoivent un grand volume de questions ou qui veulent offrir des horaires étendus en participant à un réseau collaboratif. Parlant coût, l'étape des négociations tarifaires doit être préparée sérieusement car les plans proposés peuvent être complexes, surtout pour les bibliothèques en réseau.

Le tableau 2 compare les principaux prestataires de logiciels de Q/R pour bibliothèques.

\section{Messagerie instantanée (Instant messaging, IM, chat system)}

La MI est un service de messagerie en temps réel grâce auquel les utilisateurs inscrits peuvent consulter la liste des correspondants avec lesquels 
ils sont en ligne simultanément et communiquer illico avec eux. Pour fonctionner, un service basé sur la MI requiert l'emploi d'un logiciel client qui se connecte à un serveur de messagerie instantanée. La volonté de coller aux habitudes communicationnelles des jeunes usagers et un budget limité peuvent motiver le choix de cet outil. Cependant, l'offre des logiciels de MI est déroutante car les applications les plus populaires (les gratuites) sont en perpétuel changement. D'autre part, de nombreuses configurations sont possibles ce qui complique la prise de décision. Les logiciels gratuits n'offrent pas les fonctions distinctives des logiciels évolués, mais ils ont l'avantage de la facilité et ne requièrent aucun téléchargement ou compte spécial sur l'écran de l'usager. Certains fonctionnent sans lien avec les systèmes d'opération ou les navigateurs et les usagers ont probablement une ou plusieurs de ces applications installées sur leurs ordinateurs personnels. Pour les bibliothèques, la MI présente des lacunes majeures : compiler manuellement les statistiques d'utilisation, absence d'archivage et un seul opérateur par compte.

\section{Exemples}

- Quelques outils propriétaires : AIM, Google Talk, MSN, Yahoo Messenger, Mercury messenger.

- Quelques outils en libre accès : aMSN, Exodus, Gajin, Miranda IM, Kopete, Psi.

\section{Agrégateur de MI}

Le serveur de MI est la compagnie chez qui l'utilisateur possède un compte, par exemple : AIM, Yahoo!, MSN, Google Talk. Pour ajouter à la confusion, les noms des logiciels clients sont parfois les mêmes. Habituellement, les serveurs offrent leurs propres clients propriétaires. On voit donc le cassetête pour la bibliothèque qui veut répondre à tous ses usagers, peu importe le service de messagerie utilisé. Le besoin de gérer simultanément plusieurs comptes a mené au développement de clients multi-protocoles. L'agrégateur est donc cet outil qui permet de suivre en parallèle différents comptes de MI à partir d'une seule interface. Les usagers peuvent contacter la bibliothèque à partir de leur compte de MI ouvert chez l'un ou l'autre de ces 
serveurs [Theiss-White, Dale, Fritch, Bonella, \& Coleman, 2009]. Les logiciels clients qui utilisent le Web comme plateforme sont arrivés par la suite et ont été rapidement adoptés. Le dernier développement notable est la MI intégrée (embedded). Le logiciel de MI peut désormais être enchâssé dans des pages Web grâce à l'utilisation d'un gadget logiciel (widget) qui donne accès à un bibliothécaire là où l'usager pourrait en avoir besoin au moment où il fait sa recherche [Izenstark, 2009]. Le gadget logiciel vient avec du codage qu'il suffit d'insérer dans une page HTML, c'est ce qui permet de générer une boîte de clavardage. Utiliser la MI de cette façon permet à l'usager de contacter la bibliothèque de manière anonyme, sans ouvrir un compte de MI. La boîte de clavardage est visible et aussitôt disponible pour recevoir une question [Breitbach, Mallard, \& Sage, 2009].

\section{Exemples}

- Quelques outils propriétaires : Trillian, iChat (Mac).

- Quelques outils libres : Meebo, Pidgin, Adium.

- Quelques widgets : MeeboMe, Chatango, Plugoo.

\section{voix sur IP (voice over IP)}

La voix sur IP permet d'utiliser Internet comme un téléphone, la voix étant transmise et reçue en temps réel. Les fournisseurs d'accès commencent à offrir cette technologie dans leurs forfaits, mais il existe aussi des services librement accessibles comme Skype. Populaire chez les internautes, Skype suscite la méfiance dans les institutions pour des raisons de sécurité informatique. L'application doit être installée à la bibliothèque et chez l'usager pour les appels entre ordinateurs, de plus l'utilisation de matériel comme un micro-casque est nécessaire. Par ailleurs, Kern (2009) se demande pourquoi les usagers voudraient faire usage d'un service audio sur Internet au lieu de simplement appeler depuis leur téléphone portable. Par exemple : University of Waterloo (Ontario) < http://www.lib.uwaterloo.ca/ asklib/index.html > 


\section{NOUVELLE GÉNÉRATION D'OUTILS Libraryh3lp}

Même si la MI est appréciée et facile d'utilisation, elle a ses limites. Pour aller plus loin, pour en faire une application mieux adaptée aux besoins des bibliothèques, une petite équipe américaine (bibliothécaire et concepteur de logiciel) a développé Libraryh3lp, une alternative aux logiciels de MI couramment utilisés. Ce nouvel outil est un hybride entre la MI et un logiciel commercial de référence. Libraryh3lp est un client développé en code source libre qui supporte la majorité des services de MI, avec des fonctionnalités additionnelles adaptées aux usages typiques (et nouveaux) des bibliothèques comme : création de plusieurs files d'attente, gestion des files d'attente, envoi de fichiers, transfert des questions entre opérateurs, gadget logiciel (widget), passerelle SMS. Libraryh3lp est une plateforme robuste et personnalisable qui permet à plusieurs opérateurs de travailler en simultanée [Profit, 2009]. Sa structure de coût est très abordable et l'absence d'un service de maintenance est compensée par une communauté d'entraide dynamique.

\section{Refchatter}

Cet outil commercialisé par la compagnie Altarama est bâti sur la plateforme de Libraryh3lp. Il est offert sous abonnement payant et est hébergé, ce qui libère de l'installation et de l'entretien.

\section{Micro-blog}

Twitter est le service qui symbolise le mieux le monde du micro-blogging (Pownce et Jaiku's sont moins connus). Des bibliothèques vont choisir cet outil parce c'est rapide, facile d'utilisation et personnalisable. Twitter permet d'échanger, de recevoir et de livrer en temps réel des messages, et ce, à travers différentes plateformes (Web, SMS, Facebook, appareil de poche, etc.). Le micro-blog est difficile à classer et est souvent décrit comme un type de MI. Tout comme le SMS, cette technologie impose une limite de taille du texte (140 caractères). Il s'avère utile pour attirer l'attention des usagers, pour faire la promotion d'un autre service ou pour dynamiser une FAQ. 
Par exemple : Undergraduate Library University of Illinois at UrbanaChampaign (Twitter @askundergrad).

\section{vidéos à la volée}

Jing < http://www.jingproject.com > est une application gratuite qui génère des vidéos à la volée. Au lieu de taper des instructions ou de transmettre un fichier à l'usager, le bibliothécaire crée, en quelques minutes, une capsule vidéo illustrant la démarche de recherche. Le fichier ainsi créé est déposé sur un site répertoire. Ensuite, le bibliothécaire transmet à l'usager le lien hypertexte menant à la vidéo à visionner. Les bibliothécaires de la California State University utilisent cet outil pour optimiser la transaction de référence virtuelle en enrichissant la communication textuelle d'images et de son [Breitbach, 2009].

\section{EXIGENCES LOGICIELLES ET MATÉRIELLES}

Avant d'implanter un nouveau service ou de signer un contrat avec une compagnie, il est essentiel de connaître le serveur (la plateforme) utilisé par son institution et de vérifier la compatibilité de l'outil considéré avec le réseau. Par exemple, un mur coupe-feu (firewall*) ou un bloqueur de fenêtre intruse empêcheront-ils le fonctionnement du service ? Le serveur mandataire (services proxy* utilisés dans les établissements universitaires) est-il compatible ? Des modules d'extension spécifiques sont-ils nécessaires et, si oui, pourront-ils être installés sur les ordinateurs de la bibliothèque ? Enfin, il est indispensable d'évaluer combien de temps/personne sera requis chaque année pour administrer le nouvel outil sur le serveur. Il est parfois plus rentable de choisir un système commercial qui sera hébergé chez le fournisseur.

\section{POURQUOI CONSIDÉRER LES LOGICIELS EN CODE SOURCE LIBRE ?}

Un logiciel commercial comporte des avantages considérables. En effet, une solution toute faite avec hébergement, service de maintenance et formation du personnel est tentante pour qui manque de temps et a les moyens 
de se payer un tel forfait. Pour qui a des moyens limités, une combinaison d'outil, en code source libre et de système maison est une option courante... Avant de faire ce choix, la prudence s'impose car la pratique montre que le maintien à long terme de tels systèmes peut devenir problématique. Un exemple parlant : celui du logiciel de référence en ligne " Askal ", qui a été implanté dans plusieurs BU françaises à partir de 2003 et qui n'est plus mis à jour depuis plusieurs années.

\section{Pour}

- pas d'achat, il suffit de télécharger à partir du Web ;

- accès au code source ce qui donne la possibilité d'adapter selon ses besoins ;

• si l'outil ne convient pas on s'en débarrasse... il n'a rien coûté.

\section{contre}

- demande du travail de programmation, de maintenance, de résolution de problèmes ;

- du personnel compétent en informatique doit être disponible ;

- pas de service de dépannage, mais une communauté d'utilisateurs à laquelle on peut recourir pour de l'aide.

Par exemple : les services L-net's, Oregon (USA) et KnowItNow's, Ohio (USA) ont été bâtis sur "Spark », un logiciel de MI en accès libre < http://www.oregonlibraries.net/spark/ >

\section{CONCLUSION}

Mettre en place un service de Q/R en ligne peut être un projet stimulant et rassembleur à condition que la démarche soit réfléchie et que les besoins aient été évalués avec réalisme. Il est tentant de lancer un nouveau service pour exploiter le dernier gadget ou logiciel à la mode. Le choix de l'outil devient alors la base décisionnelle du projet, ce qui peut mener à plus de déception que de satisfaction. Néanmoins, l'offre des outils en libre accès donne plus de latitude pour tester un service en interne ou sous forme de 
projet pilote, tout en évitant de mobiliser trop de ressources. Avec les logiciels commerciaux, la marge d'action est réduite, c'est pourquoi il ne faut pas hésiter à solliciter les compagnies pour obtenir une démonstration, une période d'essai et beaucoup d'information !

\section{TABLEAU 1 GRILLE D'ÉVALUATION DE LOGICIELS DE Q/R}

Utilisation de la grille : pondérer les caractéristiques des logiciels sur une échelle 0-4, où 0 signifie que cette caractéristique n'est pas désirée et 4 qu'elle est nécessaire. 1 à 3 sont des degrés croissants de désirabilité. Ensuite, évaluer si les logiciels rencontrent les exigences. Le système proposé utilise les symboles : +, $\sqrt{ },-$. Où + signifie que le logiciel possède la caractéristique, - que cette caractéristique est absente et $\sqrt{ }$ signifie que l'élément est présent mais qu'il ne se distingue pas ou qu'il est difficile de juger de sa performance (peu fréquent).

Source : Reproduit conformément aux dispositions de l'utilisation équitable et, ainsi, avec la permission de l'American Library Association à partir de Virtual reference best practices: tailoring services to your library par Kern Kathleen M. ; (c) 2009. 
Caractéristiques

Importance

$0=$ pas requis

\begin{tabular}{|l|l|l} 
Logiciel 1 & Logiciel 2 & Logiciel 3
\end{tabular}

4= nécessaire

\section{Exigences logicielles et matérielles}

Compatible avec la majorité des systèmes d'exploitation

Aucun plugiciel à télécharger

Utilise peu de bande passante

Hébergement local

Hébergement chez le fournisseur

\section{Apparence et convivialité}

Interface personnalisable

Formulaire pour entrer la question

Pas de compte usager à ouvrir

Facile d'utilisation

Authentification de l'usager supportée

\section{Communication}

\section{Conavigation}

Envoi/partage de fichiers

Messages préenregistrés (scripts)

Pousser des pages Web

Intégration du courrier électronique

Voix sur IP

Chat/Clavardage-vidéo

\section{Collaboration}

Transfert d'appel entre opérateurs

Chat/Clavardage entre opérateurs

File d'attente partagée

Remplaçant ou service hors horaire

\section{Administration}

Transcription des échanges sauvegardée

Rapports statistiques

Possibilité de fixer des niveaux d'accès

Promotion (personnalisation)

Prix

Commentaires

Classement 
COMPARATIF DES PRINCIPAUX PRESTATAIRES COMMERTABLEAU 2 CIAUX OU EN CODE SOURCE LIBRE DE LOGICIELS DE Q/R

\begin{tabular}{|c|c|c|c|c|c|}
\hline Principales caractéristiques ${ }^{3}$ & LiveHelper & LivePerson & QuestionPoint & VRLplus & Libraryh3lp \\
\hline \multicolumn{6}{|l|}{ Interface de l'usager } \\
\hline Compatibilité PC/Mac & oui & oui & oui & oui & oui \\
\hline $\begin{array}{l}\text { Compatibilité avec les } \\
\text { navigateurs }\end{array}$ & principaux & principaux & principaux & principaux & principaux \\
\hline Plugiciel/plug-in à télécharger & non & non & non & non & non \\
\hline Langues supportées & An, et + & An, Fr & An, Fr, et + & An, et? & An, Fr et + \\
\hline Interface de l'opérateur & & & & & Dépend du \\
\hline $\begin{array}{l}\text { Compatibilité fureteur / } \\
\text { système d'opération }\end{array}$ & $\begin{array}{l}\text { principaux } \\
\text { /PC }\end{array}$ & $\begin{array}{l}\text { IE, FireFox } \\
\text { /PC }\end{array}$ & $\begin{array}{l}\text { IE, FireFox } \\
\text { /PC }\end{array}$ & $\begin{array}{l}\text { principaux } \\
\text { /PC, Mac }\end{array}$ & $\begin{array}{l}\text { client choisi } \\
\text { /PC, Mac, Linux }\end{array}$ \\
\hline Gadget intégré (widget) & oui & $?$ & oui & non & oui \\
\hline Chat/Clavardage en direct & oui & oui & oui & oui & oui \\
\hline Messages/URL pré enregistrés & oui & oui & oui & oui & non \\
\hline Partage de fichiers/URL & oui & oui & oui & oui & oui \\
\hline Conavigation $^{4}$ & $?$ & oui & oui & oui & non \\
\hline $\begin{array}{l}\text { Appels multiples (répondre à } \\
\text { plus qu'un appel à la fois) }\end{array}$ & oui & oui & oui & $?$ & oui \\
\hline Opérateurs en simultané & oui & oui & oui & $?$ & illimité \\
\hline Gestion file d'attente (queue) & oui & oui & oui & oui & oui \\
\hline $\begin{array}{l}\text { Transfert d'appel entre } \\
\text { opérateurs }\end{array}$ & oui & oui & oui & oui & oui \\
\hline $\begin{array}{l}\text { Surveillance des appels } \\
\text { (Chat monitoring) }\end{array}$ & oui & oui & oui & $?$ & oui \\
\hline IM/MI entre opérateurs & oui & oui & oui & oui & oui \\
\hline Information sur l'appel entrant & oui & oui & oui & & oui \\
\hline $\begin{array}{l}\text { Historique des échanges } \\
\text { (Session transcripts) }\end{array}$ & oui & oui & oui & oui & oui \\
\hline Rapports statistiques & oui & oui & oui & oui & oui \\
\hline $\begin{array}{l}\text { Personnalisation aux couleurs } \\
\text { de la bibliothèque }\end{array}$ & oui & oui & oui & oui & oui \\
\hline Base de connaissance & non & oui & oui & oui & non \\
\hline $\begin{array}{l}\text { Questionnaire envoyé } \\
\text { à l'usager }\end{array}$ & oui & oui & oui & oui & non \\
\hline $\begin{array}{l}\text { Module de courrier } \\
\text { électronique intégré }\end{array}$ & oui & oui & oui & oui & non \\
\hline Hébergement & oui & oui & oui & oui & oui/client Web \\
\hline Autres & $\begin{array}{l}\text { Prise de } \\
\text { contrôle de } \\
\text { l'écran de } \\
\text { l'usager, } \\
\text { Voix sur IP, } \\
\text { Surveillance } \\
\text { du trafic sur } \\
\text { le site }\end{array}$ & $\begin{array}{l}\text { Voix sur IP } \\
\text { intégrée }\end{array}$ & $\begin{array}{l}\text { Quidget pour } \\
\text { service de } \\
\text { référence } \\
\text { mobile (SMS) }\end{array}$ & $\begin{array}{l}\text { Produits } \\
\text { associés : } \\
\text { RefTracker, } \\
\text { RefChatter }\end{array}$ & $\begin{array}{l}\text { Plusieurs files } \\
\text { d'attentes } \\
\text { permises, } \\
\text { Passerelle } \\
\text { SMS, Twitter } \\
\text { intégré. Code } \\
\text { source libre }\end{array}$ \\
\hline
\end{tabular}




\section{ENCADRÉ BIBLIOTHĖQUES UTILISANT LES LOGICIELS PRÉSENTÉS}

\section{Logiciel LiveHelper < http://www.livehelper.com >}

State Library of Iowa < http://www.statelibraryofiowa.org/services/askalibrarian/ >

North Idaho College Workforce Training Center < http://workforcetraining.nic.edu >

\section{Logiciel Liveperson pro < http://solutions.liveperson.com >}

Massachusetts College of Pharmacy and Health Sciences < http://www.mcphs.edu/libraries/ >

Ask0ntario < http://askon.ca/fr/index.shtml >

American Intercontinental University (sur la page d'accueil) < http://www.aiuonline.edu >

Universitätsbibliothek Dortmund < http://www.ub.uni-dortmund.de/literatursuche/auskunftonline.html > Open University Library < http://library.open.ac.uk/about/index.cfm?id=6939 >

\section{Questionpoint < http://www.questionpoint.org >}

Enssib < http://www.enssib.fr/questions-reponses/ >

BiblioSésame < http://www.bibliosesame.org/WD140AWP/WD140AWP.exe/CONNECT/BiblioSesame >

Rue des Facs < http://www.ruedesfacs.fr >

Ubib.fr < http://www.ubib.fr >

SCD Reims < http://www.univ-reims.fr >

Université Concordia < http://library.concordia.ca/help/questions/ >

Pregunte < http://www.pregunte.es >

Cornell University Library < http://www.library.cornell.edu/ask/ > ; guide pour le personnel :

$<$ http://www.library.cornell.edu/olinuris/ref/chat.htm >

People's Network (service "Enquire") < http://www.peoplesnetwork.gov.uk >

\section{VRLplus < http://www.altarama.com/page/VRLplus.aspx >}

DePaul University Libraries < http://vrlplus.cb.docutek.com/depaul/vrl_entry.asp >

Columbia College Chicago < http://vrlplus.cb.docutek.com/colum/vrl_entry.asp >

New England Law Library < http://vrlplus.cb.docutek.com/nesl/vrl_entry.asp >

Fråga biblioteket (Suède) < http://www.fragabiblioteket.se >

\section{Libraryh3lp < https://libraryh3lp.com/docs/ >}

K-State Libraries < http://www.lib.k-state.edu/reference/vref/index.html >

University of North Carolina at Chapel Hill < http://www.lib.unc.edu/ask.html >

University of Western Ontario (Canada) < http://www.lib.uwo.ca/taylor/researchhelp.shtml >

Source : le site Web Library Success Wiki < http://www.libsuccess.org >

3. Les informations qui apparaissent dans ce tableau ont été recueillies sur les sites Web des prestataires. Des renseignements plus détaillés pourront être obtenus en communiquant directement avec ces derniers.

4. La conavigation est une caractéristique qui fonctionne en principe. Des problèmes de performance et de compatibilité ont historiquement été rapportés par les utilisateurs. 


\section{5}

COMMENT ÉVALUER UN SERVICE par Nicolas Alarcon DE QUESTIONS-RÉPONSES EN LIGNE ?

L'évaluation d'un service de questions-réponses en ligne est nécessaire pour au moins deux raisons. D'une part, certains services utilisent des modes de renseignement innovants (chat, vidéo) sur lesquels nous avons encore peu de recul ; d'autre part, l'investissement financier et en ressources humaines peut s'avérer important, les données produites par l'évaluation permettront de mesurer l'activité du service et d'en ajuster l'organisation si nécessaire.

Encore faut-il savoir quoi évaluer. Les points sur lesquels peut porter l'évaluation sont nombreux : aspects économiques (coût, rentabilité et productivité du service), procédures (efficacité et efficience des processus, aspects du système utilisé et interactions avec l'usager), ressources (livres, périodiques, index, bases de données, équipements et interfaces des environnements physiques ou électroniques), résultats (qualité des réponses, informations ou connaissances délivrées à l'usager), satisfaction (degré à partir duquel l'usager du service se déclare satisfait des modalités et des résultats, satisfaction du bibliothécaire), etc.

Évaluer nécessite du temps et peut s'avérer coûteux en cas d'utilisation de certaines méthodes d'enquête ; il est donc rare de pouvoir tout explorer. En fonction des caractéristiques du service de Q/R et des exigences des décideurs, le plan d'évaluation du service opérera une sélection parmi les éléments à mesurer. Plus le plan sera pensé en amont lors de la construction du service, plus il intégrera naturellement son fonctionnement quotidien.

\section{PILOTER L'ACTIVITÉ}

La première évaluation doit concerner l'activité du service. Un tableau de bord mis à jour régulièrement synthétisera les principaux indicateurs quan- 
titatifs. La liste de ces indicateurs dépendra des modes de questionnement proposés et de la méthode de production de ces données (recueil automatique via l'application qui gère le service ou recueil par échantillonnage et analyse des questions/réponses). Voici une énumération non-exhaustive des indicateurs quantitatifs pouvant refléter l'activité d'un service de Q/R :

\section{Général}

- nombre total de questions reçues (indépendamment du mode d'interrogation utilisé) ;

- répartition des questions reçues par mode de questionnement (e-mail, sessions de chat, session de vidéoconférence, etc.) ;

- répartition par type de questions (questions pratiques, bibliographiques, de localisation, d'aide à l'utilisation des ressources électroniques, etc.) ;

- répartition des questions reçues selon la discipline concernée ;

- répartition des questions reçues par données signalétiques requises lors de la soumission de la question : origine géographique ou université d'appartenance de l'usager, sexe, statut, profession et catégorie socioprofessionnelle, etc.

\section{Service asynchrone (formulaire Web, e-mail, forum)}

- nombre moyen/médian de questions traitées par bibliothécaire ;

- temps moyen/médian passé par le bibliothécaire à effectuer les recherches pour répondre à une question ;

- durée moyenne/médiane de traitement d'une question (temps entre la réception de la question et l'envoi de la réponse).

\section{Service synchrone (chat, vidéoconférence)}

- durée moyenne/médiane d'une session de chat ou de vidéoconférence ; 
- répartition des sessions de chat ou de vidéoconférence par jour de la semaine et heure de la journée.

\author{
Base de connaissances (archive des questions et des réponses consul- \\ table librement par le public) \\ - Nombre de Q/R dans la base \\ - nombre de consultations de la base de connaissances ; \\ - nombre moyen/médian de consultations par question.
}

En matière d'indicateurs quantitatifs, les normes internationales favorisent l'utilisation d'une terminologie et d'une méthodologie commune. La mesure de l'activité des bibliothèques est abordée dans deux normes publiées par l'Organisation internationale de normalisation (ISO) : ISO 2789 Statistiques de bibliothèques et ISO 11620 Indicateurs de performance en bibliothèque. Ces documents définissent les statistiques produites dans les bibliothèques (municipales, universitaires, nationales) de manière fiable et dans une perspective internationale.

La norme ISO 2789 propose une définition restrictive et légitimiste de l'unité de mesure d'un service de Q/R : la demande d'informations. Elle est une " opération de médiation auprès du public par le personnel de la bibliothèque à partir d'une ou de plusieurs sources d'informations [...]. La demande peut être adressée de vive voix, par téléphone, par courrier, par télécopie ou par voie électronique (messagerie électronique, site Web, de la bibliothèque ou tout autre moyen de communication en réseau) [...] ». Si la définition intègre les demandes bibliographiques et les instructions concernant l'utilisation des ressources électroniques, elle exclut explicitement « les demandes de renseignement ou d'orientation et les questions administratives, telles que trouver un agent de la bibliothèque ou repérer des équipements, préciser les horaires d'ouverture ou expliquer le fonctionnement des équipements, imprimantes ou ordinateurs [...], les questions relatives à la localisation de documents du fonds déjà identifiés dans le catalogue ». Il est donc difficile de rendre compte de l'activité d'un service de Q/R en se basant littéralement sur la statistique " Demande d'informations », une part non négligeable de questions ne serait pas comptabilisée. Cette définition pourrait être élargie pour mieux prendre en compte la réalité des services électroniques 
suite à la révision de la norme ISO 2789, entreprise en 2010, la dernière version datant de 2006.

La norme ISO 11620 présente, notamment, un indicateur de performance pertinent pour les services de Q/R. L'indicateur Pourcentage des demandes d'information transmises par voie électronique permet de mesurer «l'utilisation des moyens électroniques de communication (par exemple courriel, référence numérique) pour transmettre des demandes d'informations » et peut être utilisé pour déterminer la prépondérance du renseignement en ligne sur les autres modes de renseignement (physique, par courrier, par téléphone, etc.).

Afin de refléter la capacité d'innovation des bibliothèques sur le modèle de l'enquête allemande BIX, l'enquête statistique générale des bibliothèques universitaires (ESGBU) propose depuis 2007 une entrée « Service de référence en ligne : nombre de questions ». Cet indicateur concerne explicitement les services de renseignement virtuels organisés et présentés comme tels par les établissements ; les adresses e-mail de contact de la bibliothèque sont exclues du comptage. Il est donc encore peu renseigné actuellement.

\section{ÉVALUER LA QUALITÉ DU SERVICE}

Une fois en place la mesure de l'activité du service, la deuxième étape consiste à en évaluer la qualité. Il convient de s'interroger sur ce que peut représenter la qualité d'un service de renseignement en ligne.

La satisfaction immédiate de l'usager quant à son utilisation du service est une première piste. Chaque réponse à une question, chaque session de chat ou de vidéoconférence sera accompagnée d'un questionnaire de satisfaction visant à évaluer dans quelle mesure l'usager est satisfait de son expérience. Le questionnaire sera volontairement court et cadré (pas plus de 5 questions dont une grande majorité de questions fermées) et il devra obligatoirement intégrer une question sur la volonté de l'usager d'utiliser à nouveau le service de $Q / R$ en ligne. En effet, la réutilisation du service représente une manifestation concrète de la satisfaction de l'utilisateur plus significative qu'une position sur une échelle d'appréciation (très satisfait, plutôt satisfait, etc.). 
La satisfaction de l'usager peut également se mesurer en différé. Le questionnaire soumis immédiatement après l'échange évalue la qualité de l'interaction entre le répondant et le demandeur et non la satisfaction de l'usager lors de l'utilisation effective des informations communiquées. Les données étaient-elles pertinentes au regard de la demande ? La démonstration de la base de données était-elle assez claire pour reproduire la recherche facilement? Ces questions peuvent trouver leur réponse grâce à un questionnaire envoyé à tous ou à un échantillon d'usagers quelques jours ou semaines après l'utilisation du service. Ce serait également l'occasion d'estimer si le service a répondu aux attentes de l'utilisateur, élément auquel la simple mesure de la satisfaction ne permet pas de répondre. Si l'appréciation de l'usager est un élément nécessaire à l'estimation de la qualité d'un service de $Q / R$, elle n'est pas suffisante, bien d'autres facteurs entrent en compte.

Pour McClure et Lankes, la qualité du service dépend de la satisfaction de l'utilisateur, mais aussi de la courtoisie (attitude des bibliothécaires), de l'exactitude (justesse des réponses fournies par les équipes), des habitués (pourcentage d'usagers qui utilisent plusieurs fois le service), de la connaissance du service (population connaissant son existence) et du coût (coût par interaction).

D'autres experts ont défini une dizaine d'indicateurs permettant d'évaluer la qualité d'un service de Q/R en ligne, parmi ceux-ci l'accessibilité (disponibilité via le Web), la rapidité de la réponse (100\% des réponses dans un laps de temps de 1 ou 2 jours ouvrés), une politique de service claire (de la procédure pour poser une question aux types de réponses fournies), l'interactivité (entretien et réponse en temps réel), l'autorité (des experts qui peuvent répondre aux questions et indiquer des ressources en ligne à consulter), la confidentialité (tous les échanges sont confidentiels), la critique et l'évaluation (une procédure régulière pour vérifier la satisfaction des usagers et du personnel), la communication sur le service (informer les usagers potentiels de la valeur du service pour leurs travaux).

En l'absence de tout référentiel sur les services de Q/R en ligne (un futur projet pour le groupe Qualibib ?), les responsables de ces services s'intéressant à la qualité devront se positionner par rapport aux recommanda- 
tions existantes, pour le moment largement anglo-saxonnes et se comparer aux autres services d'une même zone géographique.

\section{ÉVALUER LA QUALITÉ DES RÉPONSES}

La qualité du service se mesure également à la qualité des réponses produites. La Reference and User Services Association (RUSA) a diffusé en ligne un guide pratique traitant de la manière de proposer un service de renseignement de qualité au travers de l'interaction avec l'usager.

\section{Quelques-uns de leurs conseils:}

- facilité d'accès : l'usager doit trouver facilement le service (présence visible partout où cela est nécessaire et spécialement en ligne) ;

- intérêt manifeste dans l'interaction : l'usager doit devenir le centre de l'attention du répondant (maintenir un contact écrit, répondre dans les délais) ;

- écoute et questionnement : identification des besoins de l'usager sans interrogatoire intrusif (écoute attentive et cordiale, questionnement pertinent) ;

- recherche : stratégie de recherche adaptée à la demande et démarche pédagogique ;

- suivi : assurer le suivi de la question jusqu'à ce que l'usager soit satisfait de la réponse apportée (poursuivre les recherches hors session, orienter vers d'autres bibliothèques, encourager l'usager à revenir au besoin).

Comment évaluer la qualité des réponses dans un service de Q/R en ligne ? Une des nombreuses méthodes existantes concilie fiabilité des données produites et coût raisonnable de production. Durant une période de référence déterminée (au minimum 2 à 3 semaines), toutes les sessions seront codées simultanément par deux bibliothécaires afin d'éviter toute perception biaisée des questions. Le codage portera sur le type d'usager, le type de question et le type de traitement. Si la catégorisation des usagers ne pose, a priori, pas de problèmes particuliers, il conviendra de définir avec précision les types de question (orientation, renseignement rapide, recherche approfondie, localisation par exemple) et les types de traitement comme inter- 
prétation de la réponse apportée (exact et complet avec références citées, exact et complet sans références citées, exact sans citation requise, inexact avec références citées fausses, redirection, etc.). Le pourcentage de réponses exactes constituera un indicateur de qualité du service. Cette analyse pourra être renouvelée régulièrement afin de mesurer l'évolution de la qualité des réponses.

\section{ÉVALUER LA NOTORIÉTÉ D'UN SERVICE DE Q/R EN LIGNE}

Lorsque d'importants efforts marketing sont consentis, notamment financiers, les décideurs souhaitent parfois avoir un retour sur la notoriété du service auprès des publics-cibles. La notoriété peut s'appréhender comme un indicateur composite qui agrège des notions quantitatives et qualitatives comme la perception, la reconnaissance ou le taux de pénétration. La notoriété d'un service de Q/R peut se mesurer à l'aune des autres modes de renseignements, quelle est la part du renseignement en ligne par rapport au renseignement «traditionnel " ? L'indicateur Pourcentage des demandes d'information transmises par voie électronique issu de la norme ISO 11620 Indicateurs de performance en bibliothèque permet d'évaluer ce rapport et ainsi déterminer le taux de pénétration d'un service de Q/R en ligne. La reconnaissance du service peut se calculer grâce à un questionnaire administré à un échantillon du public potentiel (utilisateurs et non-utilisateurs) en leur demandant s'ils connaissent le service proposé et sa principale fonction.

Enfin, la perception d'un SRV est une donnée qualitative, difficilement quantifiable par un ratio ou un pourcentage. La méthode des entretiens collectifs (ou focus groups) pourra être avantageusement utilisée pour déterminer la manière dont est perçu le service par les usagers ou les non-usagers et ainsi adapter l'offre de service.

\section{L'AVIS DES BIBLIOTHÉCAIRES}

Pour être complète, l'évaluation doit concerner tous les acteurs d'un service. Parce que les services de Q/R recrutent encore le plus souvent sur la base du volontariat, l'avis des bibliothécaires sur leur participation au service 
est également à prendre en compte. Une enquête par questionnaire pourra être l'occasion de mesurer leur satisfaction à participer aux différents modes de renseignement en ligne, mais aussi de proposer des idées d'améliorations ou de recenser les besoins en formation soulevés par cette nouvelle activité.

\section{QUELS OUTILS ?}

Si l'application informatique sur laquelle se base le service de Q/R ne propose pas de rapports statistiques élaborés, l'activité pourra être comptabilisée sur un simple logiciel de bureautique : tableur (Calc®, Excel@) ou base de données (Access $\mathbb{C}$, FileMaker Pro(C). Concernant les enquêtes par questionnaire, il existe une grande variété d'outils permettant de réaliser, d'administrer et de diffuser des sondages en ligne. Au-delà des logiciels payants (Sphinx, Modalisa), des applications gratuites et/ou libres sont disponibles sur le marché telles que Googles Docs (permet de créer une enquête via un formulaire Web qui alimente automatiquement un tableur) ou Lime Survey (outil open source de création et de traitement d'enquête).

\section{AMÉLIORER LE SERVICE}

Pilotage de l'activité, mesure de la qualité, enquêtes auprès des acteurs, autant de mesures évaluatives ne sauraient être produites en ne jouant qu'un rôle de surveillance, sans contribuer à l'amélioration directe du service.

Ainsi, l'enquête sur la notoriété pourra entraîner un ajustement dans la stratégie marketing en direction des populations-cibles les moins touchées (nouveaux supports de communication ou adaptation du message). Les statistiques d'usages étayeront les probables ajustements de l'organisation du service : doublement des permanences de chat à certaines heures de la journée, renforcement ou diminution des équipes de répondants en cas de trop fréquentes ou de trop rares sollicitations, meilleur découpage thématique pour éviter un trop grand déséquilibre entre les catégories, etc. L'analyse des questions et des réponses constituera, sans aucun doute, la plus importante source d'améliorations potentielles aussi bien pour le service 
en ligne que pour l'établissement physique. Les échanges usager-bibliothécaire par e-mail ou chat du service Ubib.fr ont, par exemple, servi de base au prestataire pour la construction de la formation au savoir-répondre ${ }^{1}$. Les signalétiques défaillantes dans les espaces des bibliothèques et les informations manquantes sur les sites Web pourront être repérées par la récurrence de certaines questions (notamment pratiques). L'université de Guelph (Canada) a analysé 600 retranscriptions de sessions de chats pour créer des FAQ et des tutoriels adaptés à la terminologie employée par les étudiants, proposer un service de renseignement mobile, améliorer la signalétique dans la bibliothèque ou ajuster leurs collections papier.

L'ouverture du service, même après plusieurs mois de réflexion et de préparation, ne constitue pas le terme de son évolution. Au contraire, toutes les données issues de l'évaluation serviront à le transformer pour répondre encore mieux aux attentes des usagers. Par son intérêt stratégique pour les responsables et décideurs du SRV, le tableau de bord synthétisant les principaux indicateurs quantitatifs est la première action d'évaluation à mettre en place. Les autres éléments (notoriété, qualité des réponses, satisfaction des usagers) ne doivent pas être négligés pour autant, malgré des méthodes de production de données plus longues et parfois plus complexes. Ils compléteront les chiffres de l'activité du service et contribueront à en restituer un portrait fidèle en multipliant les éclairages sous différents angles. 


\section{PARTHE TII}

\section{ORGANISER SON SERVICE}

1. COMMENT S'ORGANISER EN INTERNE ; LE SAVOIR-ÊTRE DU RÉPONDEUR par Sandrine Lebastard et Tù-Tâm Nguyên $+++++++++++++++++++++++++++++++++++$

2. TRAVAILLER ENSEMBLE : L'EXEMPLE D'UBIB.FR par Nadine Kiker

3. RUE DES FACS

par François Michaud

4. LE SI@DE, UN LABEL ET UN RÉSEAU DE COOPÉRATION ENTRE SERVICES par Isabelle Copin 


\section{1}

COMMENT S'ORGANISER EN INTERNE ; LE SAVOIR-ÊTRE par Sandrine Lebastard et DU RÉPONDEUR Tù-Tâm Nguyên

\section{LES COMPÉTENCES À ACQUÉRIR, LES FORMATIONS À METTRE EN CEUVRE}

\section{ACQUÉRIR LES COMPÉTENCES DE RÉPONDEUR'}

Savoir :

- répondre à l'usager Formation 1 ;

- rechercher l'information ${ }^{\text {Formation } 1}$;

- quelle stratégie appliquer (équipe de répondeurs ou réseau des partenaires...) ${ }^{\text {Formations } 2 \text { et } 5}$;

- évaluer et sélectionner les sources :

- pertinence, fiabilité, complémentarité entre elles, ${ }^{\text {Formation } 1 \text {, }}$

- adéquation du niveau des informations par rapport au

demandeur et aux lecteurs potentiels du service Formations 2 et 3 ;

- contribuer à, valider, mettre à jour la base de connaissances Formation 4 ;

- alimenter et mettre à jour la base interne d'outils ${ }^{\text {Formation } 4}$;

- faire des suggestions de forme et de fond de façon constructive et bienveillante Formations 2 et 5 .

\section{DÉFINIR LES TÂCHES DU COORDINATEUR DU SERVICE}

Il intervient à plusieurs niveaux, sur le plan :

- technique : il assure le suivi du logiciel utilisé (évolution de l'outil, développements nécessaires, relation avec le service informatique et le prestataire...) ;

- du processus de travail : il veille à la cohérence des réponses et au respect des délais, planifie la présence de répondeurs... ; 
- marketing : il impulse la communication envers le public visé, entretient et développe les relations avec les partenaires, encourage les échanges avec les autres services de réponses...

\section{HOMOGÉNÉISER LES COMPÉTENCES DE L'ÉQUIPE DE RÉPONDEURS}

Définir en commun la charte interne :

- dégager les valeurs communes et les stratégies de réponse préférentielles comme par exemple, reformuler ou non la question, limiter le nombre de références, expliciter la méthodologie de recherche, rédiger ou adopter un style télégraphique, donner quelques pistes de recherches ou répondre précisément à la question... ${ }^{\text {Formation } 5}$;

- préciser les limites du service rendu : par exemple, se donner des repères quantitatifs en termes de nombre de citations, longueur de réponse, temps passé... Formation 5 ;

- rédiger des scripts de réponses (réponses standards pour les messages répétitifs) comme les formules de politesse, comment utiliser l'OPAC... ${ }^{\text {Formations } 2 \text { et } 3}$;

- travailler de façon collaborative (ne pas hésiter à échanger doutes et trouvailles entre répondeurs, accepter d'être relu et critiqué sans craindre d'être remis en cause) ${ }^{\text {Formation } 5}$;

Programmer des réunions de débriefing régulières, se donner du recul pour :

- échanger sur les difficultés rencontrées ;

- mutualiser les solutions, les outils, les ressources ;

- statuer sur les modes de traitement à appliquer pour faire face à des situations nouvelles (les questions à la frange des domaines traités par le service, multiplication d'un type de questions qui n'est pas le cœur de cible du service...).

Ces réunions participent de la formation continue de l'équipe. 


\section{ENCADRÉ 5 TYPES DE FORMATIONS À METTRE EN GEUVRE}

Formation 1 : rechercher l'information spécialisée sur Internet et optimiser l'interrogation des bases de données spécialisées

Deux aspects sont à distinguer :

- il faut trouver les sources pertinentes, les informations fiables pour chaque réponse. Il est donc nécessaire de connaître les ressources spécialisées des domaines traités. Une formation sur les sources thématiques doit être envisagée si cela n’a pas été déjà fait pour le service en présentiel. Cela permettra en outre de constituer un répertoire de signets soit accessibles au public soit en interne ;

- afin d'améliorer la qualité des recherches, il est indispensable de connaître la méthode d'interrogation propre à chaque grande banque de données (comme Pubmed pour le domaine médical).

Formation 2 : bien répondre à l'internaute

Les répondeurs travaillent souvent dans l'urgence. Il est d'autant plus nécessaire de les sensibiliser à une lecture attentive de la question, surtout si celle-ci est à forts enjeux émotionnels. Suivre une formation qui permette de prendre conscience de la nécessité de ce recul et favorise l'empathie avec l'internaute est indispensable.

Formation 3 : écrire pour être lu sur le Web

Répondre en ligne nécessite une maîtrise de la rédaction spécifique au Web : écriture plus synthétique, plus structurée...

Formation 4 : maîtriser l'outil informatique $^{2}$ :

Quel que soit l'outil utilisé, une formation sur l'outil utilisé est indispensable. Cette formation doit être renouvelée au fur et à mesure de l'évolution de l'outil. Le coordinateur du service devra avoir une formation d'administrateur. Il est utile de nommer un suppléant.

Formation 5 : assistance à la maîtrise d'ouvrage

Pour concevoir et mener à bien le projet, s'adjoindre les services d'un consultant pour assistance à maîtrise d'ouvrage peut s'avérer très fructueux (élaboration de la charte du service, choix éventuel du nom du service, benchmarking...).

2. Cf. l'article d'H. Tardif, « Quels outils pour les services ? Quelle technologie choisir ? », p. 94. 


\section{IDENTIFIER LES FORMATIONS À METTRE EN PLACE}

Les formations qui portent sur les contenus documentaires (connaissance thématique, connaissance des outils de recherche) sont communes aux services en présentiel et virtuel. Les services de $Q / R$ en ligne requièrent un savoir-faire et un savoir-être particulier pour la relation à l'autre à distance, pour la maîtrise de l'écrit et de l'outil informatique (par exemple, des formations spécifiques au chat peuvent être organisées).

Des formations peuvent être envisagées avant le lancement du service puis au cours de la première année lorsque la pratique met à jour certains besoins, communs à toute l'équipe ou nécessaires à quelques répondeurs en particulier. Certaines formations, notamment celles qui concernent le savoir rédiger ou l'écriture sur le Web, gagnent à être fractionnées dans le temps. En effet, entre deux sessions, les répondeurs peuvent mettre en pratique les recommandations préconisées en formation et lister les écueils rencontrés afin de les soumettre au formateur. Celui-ci pourra alors adapter le contenu de la prochaine formation en fonction des besoins exprimés. Les formations à la recherche sur Internet et à l'interrogation des banques de données doivent être réactualisées au moins tous les 2 ans.

Pour tous les besoins de formation spécifiques, il ne faut pas hésiter à rédiger un cahier des charges propre au service et à mettre en concurrence les prestataires de formation si leurs offres standards ne conviennent pas tout à fait.

\section{LE CIRCUIT DE TRAITEMENT DE LA QUESTION, LE PROCESSUS DE TRAVAIL}

\section{RÉCEPTION DE LA QUESTION}

Elle peut arriver dans une BAL qui doit être ouverte plusieurs fois dans la journée afin de limiter au maximum le temps de latence. Elle peut arriver aussi sur une interface dédiée, avec un système d'alerte e-mail. Selon l'organisation, cette étape peut incomber au coordinateur ou à l'ensemble des répondeurs.

Si la question arrive par e-mail, le répondeur crée un fichier de traitement de texte par question. Dans le nom du fichier sont indiqués la date de récep- 
tion, le sujet et un code indiquant si la réponse pourra être publiée ou non. Ce fichier est placé dans un dossier identifié sur le réseau interne accessible à tous les répondeurs.

Dès qu'une nouvelle question est prise en charge par un répondeur, quand ce n'est pas automatique, il avertit ses collègues. Il est judicieux d'informer le lecteur que sa question a été reçue et va être traitée dans les délais (sinon, expliquer pourquoi et donner un nouveau délai), avec un numéro de « ticket » pour garantir sa traçabilité.

S'il s'agit d'une question posée par chat que le bibliothécaire ne peut prendre en charge immédiatement, il convient d'indiquer à l'internaute, le temps d'attente estimé, pour le faire patienter. Certains logiciels gèrent les files d'attente virtuelles.

\section{TRAITEMENT DE LA QUESTION}

Autant que possible, il est préférable de traiter les questions par ordre d'arrivée pour faciliter le respect des délais affichés, sauf quand une réponse urgente est nécessitée. Il faut détailler dans la charte interne la prise en charge de la question, et le niveau de priorité le cas échéant.

\section{Lecture attentive de la question}

Cette première étape est primordiale et requiert une grande attention pour :

- éviter toute interprétation erronée de la question ;

- dégager la question précise d'une thématique plus large ;

- distinguer les différents points soulevés par la question, une question pouvant en englober plusieurs ;

- déterminer les enjeux en question (économiques, juridiques, professionnels, personnels...) ;

- déterminer le profil et le niveau de connaissance de l'internaute et adapter le niveau de réponse en conséquence.

\section{Recherche des informations}

La recherche des informations pour un service en ligne requiert intuition et méthode. Pour le différé, la difficulté de ne pas pouvoir interagir en direct avec un interlocuteur physique est compensée par le temps laissé pour 
développer toutes les étapes logiques d'une recherche. La réponse écrite qui laisse une trace - exige une rigueur particulière.

\section{Il faut penser à :}

- interroger la base de connaissances pour bénéficier des recherches déjà effectuées pour une question similaire ou proche ;

- chercher des informations sur les sites généralistes de référence pour avoir une première approche du sujet (si on ne le connaît pas) afin de s'orienter vers les sites spécialisés adéquats ;

- cibler la recherche en fonction de la question sur des sources plus spécialisées : sitothèque, base outils interne pour les répondeurs (wikis, blogs internes...).

Tous les liens repérés sont conservés soit avec une courte description du contenu soit avec des extraits répondant à la question, de façon à faciliter la rédaction.

Les sources de qualité repérées lors de cette recherche seront capitalisées dans la base outils.

Pour le chat, c'est la quasi-immédiateté qui prévaut... Si nécessaire, on indiquera à l'internaute qu'on prendra en charge la réponse ultérieurement.

\section{Rédaction de la réponse ${ }^{3}$}

\section{Relecture collective de la réponse}

Pour vérifier la pertinence de la réponse, une relecture collective est souhaitable (au minimum par une deuxième personne), voire une co-écriture pour les questions complexes. La relecture finale peut être faite par le coordinateur. Ce regard croisé permet d'éviter divers écueils comme d'avoir traité un sujet sans répondre à une question précise, avoir utilisé un langage spécialisé sans avoir explicité les principaux termes...

3. Cf. l’article de M.-F. Defosse, « Comment répondre et qu'est-ce que bien répondre ? Construire le savoirrépondre », p. 78. 


\section{Relecture personnelle « méta » de la réponse in fine}

Le répondant doit aussi s'interroger sur ce qu'il pourrait souhaiter transmettre de cette expérience (difficultés rencontrées, découvertes...) au prochain débriefing.

\section{Saisie de la question-réponse dans l'outil informatique}

Une fois la réponse modifiée et validée, la dernière étape consiste à la saisir dans l'interface. Elle est envoyée par e-mail à l'internaute et publiée si l'autorisation de publication a été donnée. Une ultime relecture attentive, voire une impression, permet de déceler les coquilles difficiles à détecter sur écran.

\section{LA BOÎTE À OUTILS À CONSTITUER, EXPLOITER, METTRE À JOUR}

"Les bons outils font les bons ouvriers ». Pour ne pas contredire cet adage, mieux vaut se constituer une belle boîte à outils qui peut comprendre :

\section{LA BASE DE CONNAISSANCES 4}

\section{LES RESSOURCES PHYSIQUES DE LA BIBLIOTHĖQUE}

Même si le renseignement en ligne nous projette dans l'univers du virtuel, n'oublions pas d'utiliser les fonds propres de la bibliothèque : livres, périodiques, films, archives, littérature grise... Ils sont souvent précieux pour démarrer une recherche, pour pallier les lacunes du Web sur certains sujets (surannés, régionaux, très spécialisés...) ou pour inviter les internautes à venir à la bibliothèque. Et puis, tout n'est pas en ligne...

\section{LES RESSOURCES NUMÉRIQUES DE LA BIBLIOTHĖQUE}

Ressources en ligne et produits documentaires élaborés par la bibliothèque ne doivent pas être oubliés.

Abonnements en ligne, sitothèque, dossiers documentaires, bibliographies sont à privilégier car ils résultent d'une sélection qualitative ou d'un travail de la bibliothèque.

4. Cf. l'article de M. Montano, « Offrir une base de connaissances : pourquoi, pour qui, comment ?», p. 89. 
Exemple : La Cité de la santé propose une sélection de plus de 250 sites médicaux, véritable réservoir pour le service Questions-santé.

\section{LE FONDS DES USUELS DE RÉFÉRENCE POUR LES RÉPONDANTS}

Ne pas oublier de constituer un fonds d'ouvrages exclusivement réservés aux répondants (ouvrages de référence, etc.), comme pour le service de référence physique !

\section{LA BASE INTERNE D'OUTILS POUR LES RÉPONDANTS}

Il est utile de regrouper dans une même base de données des objets informationnels repérés lors des recherches et susceptibles d'être réutilisés. Sites Internet, institutions, associations, services téléphoniques, lieux d'information, brochures papier, guides, fichiers PDF, vidéos en ligne... sont indexés et qualifiés par niveau de public avec un court descriptif. Pour les services collaboratifs, elle permet aussi de lister les ressources auxquelles sont abonnés les participants.

Cette base doit être alimentée de manière courante au cours de chaque recherche et revue périodiquement pour vérifier l'actualité des informations.

Elle peut prendre la forme d'un wiki comme à la Bpi, ou d'un blog comme pour Ubib.fr ${ }^{5}$.

\section{POURQUOI DÉVELOPPER DES PARTENARIATS ?}

Afin de répondre au mieux aux internautes, il est indispensable de s'entourer d'experts qui, soit à titre personnel, soit au titre de leur organisme ou association pourront apporter une aide pour des questions épineuses en ajoutant de la fiabilité aux réponses et en valorisant le service.

Consulter un partenaire peut paraître ralentir le circuit de traitement en ajoutant une étape, mais en fait cette démarche peut épargner beaucoup de temps. C'est pourquoi il est nécessaire qu'à l'arrivée de la question, le

5. Cf. l'article de N. Daigne, «Un réseau généraliste de questions-réponses de bibliothèques publiques : premier bilan », p. 169 et l'article de N. Kiker, « Travailler ensemble : l'exemple d'Ubib.fr », p. 130. 
répondant s'interroge sur l'utilité de cette consultation et la prenne en compte dans le délai de traitement.

\section{CONSULTER UNE PERSONNE RESSOURCE - EXPERT DU DOMAINE - EN AMONT DE LA RECHERCHE}

Peut être très utile pour :

- gagner du temps dans le décryptage d'éléments problématiques fournis par l'internaute (terminologie spécifique retranscrite avec des erreurs, séries de valeurs chiffrées difficiles à identifier...);

- avoir des conseils sur les pistes à explorer voire des éléments de réponse, comme Questions-santé procède avec la Maison des usagers du Centre hospitalier Sainte-Anne pour les maladies mentales. Dans ce cas, la contribution du partenaire sera mentionnée explicitement et légitimera la réponse.

\section{CONSULTER UNE PERSONNE RESSOURCE POUR VALIDATION DE LA RÉPONSE}

Permet de vérifier que l'on n'a pas :

- oublié une source incontournable ;

- commis une erreur d'interprétation ou un contresens en essayant de vulgariser un contenu complexe ;

- donné une réponse totalement inepte à une question délicate.

\section{RÉORIENTER LA QUESTION VERS LE PARTENAIRE LE PLUS ADAPTÉ}

Est judicieux quand :

- dans son réseau de connaissances - formalisé ou non il existe un partenaire qui possède les compétences idoines pour apporter une réponse d'excellence, comme par exemple, Maladies Rares Info Services dans le domaine des maladies orphelines.

- la question n'entre pas dans le champ thématique du service. Adhérer à un réseau de réponses permet des 
redirections de questions vers les interlocuteurs les plus adaptés quelle que soit la porte d'entrée de l'internaute.

Dans tous ces cas, il faut mettre en place une procédure de délégation du traitement (méthodes de transmission, information de l'internaute, suivi du traitement de la question et de son délai).

\section{Quelques exemples de partenariats}

Selon l'environnement du service de Q/R (université, grand établissement, musée, etc.) les partenaires peuvent varier. Ce peut être des :

-réseaux de réponses comme Si@de (services aux thématiques différentes) ;

- partenaires de la profession : centres de documentation, bibliothèques ; - partenaires des thématiques couvertes : organismes de référence, sociétés savantes, associations d'usagers... ;

- professionnels ou spécialistes du domaine ;

- partenaires internes mais relevant d'autres services (scolarité pour les SCD ; administration ; informatique...). 


\section{2 \\ TRAVAILLER ENSEMBLE : par Nadine Kiker L'EXEMPLE D'UBIB.FR}

Ubib.fr < http://www.ubib.fr >

Travailler ensemble ne se décrète pas, même dans une proximité géographique, mais se construit. La collaboration, exercice difficile, résulte d'une volonté politique forte qui s'inscrit dans des frontières précises, avec de larges ambitions, qui nourrissent la vie quotidienne d'un réseau collaboratif comme Ubib.fr, ce que nous allons tenter de montrer.

\section{DE NOUVELLES FRONTIÈRES}

Le réseau Ubib.fr procède d'abord d'une logique de conquête de nouveaux territoires. La généralisation de l'accès à Internet a bouleversé les habitudes de travail des étudiants. Cette génération $\mathrm{Y}$ de digital natives, arrivée dans nos SCD, utilise massivement des supports électroniques. Ce constat est néanmoins un élément majeur pour la construction d'un réseau de renseignement virtuel (SRV) ou de questions-réponses en ligne, puisqu'il oblige le bibliothécaire, fondamentalement médiateur, à être là où se trouve l'usager, c'est-à-dire en ligne.

Par ailleurs, la massification des ressources en ligne justifie la construction d'un SRV. En effet, depuis 10 ans, la part des ressources en ligne dans la documentation augmente sensiblement. Au SCD d'Angers, la part budgétaire de la documentation électronique sur la documentation totale est passée de 7 à $41 \%$ en 5 ans. Elle a donc été multipliée par 6 . Et proposer seulement un accès à ces nombreuses ressources ne suffit pas à leur utilisation massive.

Enfin, le travail de formation des usagers, réalisé en début de semestre, ne saurait être pleinement efficace s'il n'était accompagné d'un travail de suivi dont la réponse en ligne est une modalité forte. 
Si les usagers occupent de nouveaux territoires, celui des bibliothèques évolue aussi. Le Réseau Universitaire Ouest Atlantique (RUOA), né d'une volonté politique de rassembler autour de projets communs 9 puis 10 universités du Grand Ouest, a permis de travailler ensemble et a donc forgé entre les SCD partenaires un sentiment d'appartenance à une communauté élargie. Créer davantage de proximité en permettant aux équipes des SCD de collaborer au quotidien au sein d'Ubib.fr vient nourrir ce sentiment. Expertises et savoir-faire, différents selon la nature des SCD - mono- ou pluridisciplinaire - sont ainsi mis en commun.

Cette orientation « géopolitique » répond à la nécessité de développer une culture professionnelle adaptée. Les 7 SCD du départ sont reliés aux mêmes instances de formation régionale. Dans ce cadre-là, ils sont amenés à réfléchir au niveau de compétences souhaité et un SRV leur a semblé un excellent moyen de capitaliser les compétences de leurs agents.

Avant Ubib.fr préexistaient déjà 2 SRV par e-mail uniquement. L'un, angevin, était géré par une personne qui répondait aux questions ou les réorientait vers ses collègues. L'autre, rennais, appelé Cible, géré par une équipe de répondants, faisait le lien entre la $\mathrm{BU}$ et les bibliothèques associées. Travailler ensemble aujourd'hui permet donc d'éviter à terme un émiettement des services et de favoriser par un système plus adapté une réponse plus efficace.

Pour finir, notons que les $10 \mathrm{SCD}^{1}$ constituant Ubib.fr correspondent à une zone géographique très large allant de Limoges à Brest et de La Rochelle au Mans. Ils ont quasiment tous des antennes universitaires et des bibliothèques associées nombreuses. Cette mosaïque de micro-établissements ne facilite pas la réponse homogène mais un travail en réseau favorise des réponses pertinentes dans un périmètre clair.

1. Universités d’Angers, du Maine, de Nantes, de Limoges, de Rennes 1 et 2, de Bretagne Sud, de Bretagne Occidentale, de La Rochelle, de Poitiers. 


\section{LES AMBITIONS D'UN SERVICE DE RÉSEAU COLLABORATIF}

La volonté de conquérir de nouveaux territoires a très vite débouché sur la rédaction d'une feuille de route par les directeurs des SCD. En février 2008, le groupe de travail eut pour mission de construire en 6 mois un réseau destiné au public des universités du RUOA. La réponse à la question de l'usager devait être systématique et accompagnée d'une orientation pédagogique et méthodologique, dans un délai de 48 heures ouvrées, pour la réponse par e-mail. Le mode d'accès serait double, laissé au seul choix des usagers. Un effort particulier serait fait sur le volet synchrone avec un service ouvert 45 heures par semaine du lundi au vendredi de $9 \mathrm{~h}$ à $18 \mathrm{~h}$. Les interrogations nombreuses au sein du réseau sur la nature des questions et la légitimité d'y répondre par messagerie instantanée, ou chat, furent gommées par une feuille de route clairement affichée, véritable boussole accélérant la première mise en place d'Ubib.fr, dès novembre 2008. La structuration initiale du service contribua aussi à cette rapidité. En effet, Ubib.fr est un réseau collaboratif d'environ 130 répondants dirigé par un comité de pilotage (CP) composé des 10 directeurs des SCD du RUOA, administré par un groupe de travail (GT) composé de 11 coordinateurs. Le CP, qui a impulsé le service, se rencontre deux fois par an pour valider les avancées du service et les orientations à venir, comme la promotion ou l'élargissement du service, la formation des répondants.

Le GT est chargé de mettre en œuvre les orientations politiques décidées par le CP. Après un gros travail de mise en place du service, le GT veille à optimiser le fonctionnement de l'outil, actuellement QuestionPoint d'OCLC. La bonne organisation du réseau humain, la valorisation du travail des répondants, la qualité de la réponse mais aussi la visibilité et la dissémination du service lui incombent. Les coordinateurs d'établissements sont aussi tous répondants, donc impliqués au quotidien et conscients de la marge de progression d'Ubib.fr. Les répondants, eux, sont très souvent polyvalents, travaillant par e-mail et en instantané. La coordination générale a été assurée successivement par l'université de Bretagne occidentale, Angers puis celle conjointe d'Angers et du Mans. 
À la structuration claire du service s'est ajoutée très tôt la nécessité de former les répondants à un service nouveau en France. Le GT a donc réfléchi à une commande de formation avec l'objectif suivant : permettre aux répondants de construire une réponse adaptée à l'usager. Cette commande fut élaborée autour de deux compétences : l'aisance dans l'utilisation d'une plateforme technique de SRV et enfin celle dans la réponse à l'usager. Elles furent jugées primordiales, ce que confirme une récente enquête américaine auprès des répondants de SRV portant sur les compétences nécessaires pour répondre par chat.

Quatre compétences ressortent de cette enquête : non seulement une bonne connaissance des ressources du réseau et une maîtrise de la plateforme technique, mais aussi une bonne compréhension de la culture du net et une bonne gestion du stress, dans un environnement multitâches et de communication non verbale ${ }^{2}$.

La formation à la plateforme technique de QuestionPoint fut dispensée avant l'ouverture du service, par le commercial, aux membres du GT qui la relayèrent ensuite auprès de tous les répondants. Ce mode de formation peu coûteux s'avéra efficace.

Attardons-nous sur la formation au savoir-répondre, orientée chat. Nous l'avons montée entièrement avec le cabinet mfDocumentation. La formation devait être pratique, courte et efficiente et valoriser le niveau de compétences des stagiaires déjà actifs dans Ubib.fr. Concrètement, cela signifia de former d'abord 53 répondants, principalement à la réponse par chat, en 4 groupes distincts avec 1,5 jour de formation/stagiaire pour un coût de $150 €$.

À ce choix de brièveté liée à l’implication professionnelle locale des répondants s'ajouta une dernière exigence, celle de rendre le répondant auto-

2. Assessment Librarian : quelles compétences pour un service de référence par chat ? [En ligne] < http://assessmentlibrarian.wordpress.com/2008/12/12/quelles-competences-pour-un-service-dereference-par-chat/ > (consulté le 3 mai 2010). 
nome dans son activité de réponse virtuelle. Pour ce faire, la demande de mises en situation fut très forte. L'enjeu était de former en partant du terrain, fût-il virtuel, afin que chaque répondant s'approprie un style de réponse personnel mais correspondant aux lignes directrices du service, rappelé par un coordinateur présent à chaque séance. Les sessions de formation déboucheront sur l'élaboration d'un mémento de la réponse par chat, qui viendra compléter le document existant sur la réponse par e-mail.

Pour conclure, la formation à ce nouveau service, absente de l'offre standard de formations, se veut à l'image d'Ubib.fr : innovante et ambitieuse, même si l'exercice a des limites que l'animation de la vie du réseau doit dépasser.

\section{VIE DU RÉSEAU}

La vie du réseau s'organise autour d'un service mixte, reposant sur un planning synchrone et une organisation en pétales. En effet, une répartition des plages de permanence de chat est établie en fonction de la taille des SCD qui se partagent la semaine en tranches de $1 \mathrm{~h} 30$ à 2 heures de $9 \mathrm{~h}$ à $18 \mathrm{~h}$, soit 2 à 4 permanences hebdomadaires gérées par chaque SCD. Un seul bibliothécaire répond aux questions posées pendant sa permanence de chat. Il est donc polyvalent. Parallèlement, il fait partie d'un des 9 pétales disciplinaires ${ }^{3}$ et répond aux questions posées par e-mail en 48 heures ouvrées.

Chaque membre du GT administre un pétale et intervient dans le pétale divers pour les questions très locales. Bon connaisseur du fonctionnement de son SCD, il est aussi correspondant et animateur des équipes Ubib.fr. Chaque établissement organise son équipe de répondants comme il le souhaite. 
La coexistence des deux modes, asynchrone et synchrone, fait d'Ubib.fr un service complexe qui doit sans cesse capitaliser et enrichir son savoir-faire. La mise en place progressive d'une panoplie d'outils y contribue.

Pour monter le service, nous avons ouvert un wiki (pbworks), lieu de stockage de nos comptes rendus de réunions et documents de travail. Nous y trouvons par exemple, outre le planning des permanences de chat, des coordonnées pratiques sur les contacts, conditions de prêt ou encore un tableau des ressources en ligne de chaque SCD.

À cette interface d'échange et d'informations s'est ajoutée une liste de diffusion, par pétale, qui permet d'avertir de l'arrivée d'une question ou de transmettre des informations relatives à l'assignation d'une question restée sans réponse au bout de 24 heures, ou encore à la vie d'un SCD ou d'Ubib.fr. Or, malgré l'existence de ces outils, les premières sessions de formation de juin 2009 ont révélé une grande hétérogénéité dans la pratique du chat ainsi que dans la maîtrise de QuestionPoint et dans la connaissance du périmètre des établissements et du service, pourtant clairement défini dans la feuille de route.

Dans le souci constant d'une réponse en phase avec les besoins de l'usager et donc la nécessité de l'appropriation par chaque répondant d'une " culture Ubib.fr », nous avons opté pour un travail sur notre service en interne et non pour une relecture, impossible en mode instantanée de toute façon. Nous utilisons désormais Facet, un outil gratuit de gestion de signets développé par le MIT, pour entrer toutes les informations portant sur les ressources et les données pratiques des SCD, ce qui facilite la réponse par chat et en garantit la pertinence par le dynamisme des liens. À ce jour, nous avons entré 190 signets.

Parallèlement, nous avons ouvert un blog ${ }^{4}$ professionnel qui doit permettre de partager difficultés et bonnes pratiques, de faire passer de l'infor- 
mation sur son établissement et d'étayer les formations reçues. À ce jour, 32 billets ont été postés : statistiques, réunions, nouveaux entrants et ressources sont des sujets récurrents. Conçu comme un outil interne de liaison, non encore diffusé sur le Web, il compte depuis octobre cinq rédacteurs et reçoit en moyenne 380 visites par mois. Il est couplé avec le wiki qui, dans sa nouvelle version, sert au stockage des documents de travail (charte interne, guide de réponse) avec un espace dédié à chaque SCD. Cette panoplie doit contribuer à améliorer la réponse et son efficacité doit être mesurée par une évaluation rigoureuse, comme l'est le service lui-même.

L'enquête menée auprès des répondants, réalisée en novembre 2009, va dans ce sens. Elle portait notamment sur la formation au savoir-répondre. Elle a montré que les attentes étaient d'autant plus grandes et plus comblées chez les intéressés qu'ils avaient pratiqué le chat, ce qui milite pour une autonomisation de chaque répondant. Des réunions régulières avec les répondants au sein des SCD doivent être multipliées pour pallier une communication encore trop peu fluide et favoriser un débriefing des réponses.

Ubib.fr apparaît donc comme issu d'une volonté politique de franchir un nouveau cap qui s'appuie sur une structure collaborative ambitieuse étayant la vie d'un réseau évolutif. Le travailler ensemble réalisé avec Ubib.fr est exemplaire. Pour difficile qu'il apparaisse, il a des vertus indéniables. Il impose une rigueur dans le contenu et l'adéquation de la réponse. Il oblige à repenser la lisibilité de nos ressources et services. Enfin, il valorise, par une attention constante, la fonction médiatrice du bibliothécaire. Une belle aventure. 


\section{3}

RUE DES FACS par François Michaud

$\Leftrightarrow<$ http://www.ruedesfacs.fr >

\section{UN SERVICE DE QUESTIONS-RÉPONSES NÉ D'UNE GÉOGRAPHIE COMPLEXE}

Porté par 23 bibliothèques universitaires d'Île-de-France, Rue des Facs est un service de questions-réponses en ligne qui s'est, dès son origine, enrichi de la géographie documentaire complexe qui lui préexistait. Fédérant des établissements de statuts différents (SCD, SICD), de rattachements divers (enseignement supérieur, CNRS), aux multiples implantations géographiques, Rue des Facs a permis, dès sa conception, de dépasser les obstacles à la fois matériels et institutionnels qui traditionnellement brouillent la lisibilité documentaire de Paris et de l'Île-de-France.

Outil et interface uniques, articulés à un schéma de travail collaboratif, Rue des Facs vient fédérer des ressources sans interférer ni contrevenir aux logiques et à l'organisation des établissements participants. Chacun continue à se rejoindre ou à se démarquer au sein d'instances ou de groupes de travail à périmètres variables : carte documentaire du Quartier latin, achats de ressources électroniques, services interuniversitaires, pôles de recherche et d'enseignement supérieur (PRES).

\section{UN SERVICE CONSTRUIT SUR UNE LOGIQUE THÉMATIQUE}

Rue des Facs est né en 2005 de la volonté de quatre bibliothèques ${ }^{1}$ d'œuvrer en commun à un service de référence virtuel qu'ils pourraient proposer à leurs publics respectifs. La relative similarité des établissements (tous présentant une prédominance en littérature et sciences humaines) et donc

1. Les services communs de la documentation des universités Sorbonne Nouvelle-Paris 3 et Paris 4-Sorbonne, ainsi que les bibliothèques interuniversitaires de la Sorbonne et Sainte-Barbe. 
des publics concernés facilitaient cette initiative. Le positionnement de la bibliothèque interuniversitaire Sainte-Barbe, dernière née des grandes bibliothèques du centre de Paris et sa mission de complémentarité avec les établissements voisins légitimait qu'elle pilote le projet à ce stade. Chacune des bibliothèques à l'origine de Rue des Facs proposait déjà de façon traditionnelle, par le biais d'une adresse e-mail ou d'un forum, un embryon de service de $Q / R$ à distance : aide à la recherche bibliographique, réponses à des questions portant sur la disponibilité d'ouvrages comme sur le fonctionnement des établissements. Ces services n'étaient cependant pas normalisés et ne pouvaient pas être mutualisés. Par ailleurs, aucun n'était très développé ni très valorisé auprès du public. L'idée de mettre en œuvre un service de référence virtuel commun, qui offrirait l'avantage d'enrichir et dépasser ces premiers services, émergea donc assez rapidement.

Une alternative se présentait alors : la mise en œuvre d'un nouveau service dans ce contexte impliquait soit une logique géographique, soit une logique thématique. Dans le premier cas, les établissements se répartiraient les disciplines retenues pour le futur service (les répondants dans ce cas ne traitant que les questions relevant de la discipline affectée à leur établissement) ; dans le deuxième cas, de grands domaines disciplinaires seraient définis, dans lesquels s'inscriraient les répondants quel que soit leur établissement.

C'est cette dernière construction logique qui a été retenue, car elle facilite la mutualisation des ressources et des compétences. De plus, ces « bibliothèques virtuelles ", en transcendant la logique d'établissements et en regroupant toutes les compétences et toutes les ressources dans des secteurs disciplinaires, permettent une réelle approche collaborative et facilitent l'accroissement du dispositif. En effet, l'arrivée d'un établissement dans le service de référence ne remet pas en question l'équilibre général mais accroît l'équipe des répondants qui se répartissent dans les disciplines qu'ils auront choisies. Dix grands secteurs disciplinaires ont ainsi été définis, et l'architecture collaborative qu'ils insèrent relève de l'arborescence en pétale. 


\section{GRAPHIQUE UNE ARCHITECTURE EN PÉTALES THÉMATIQUES}

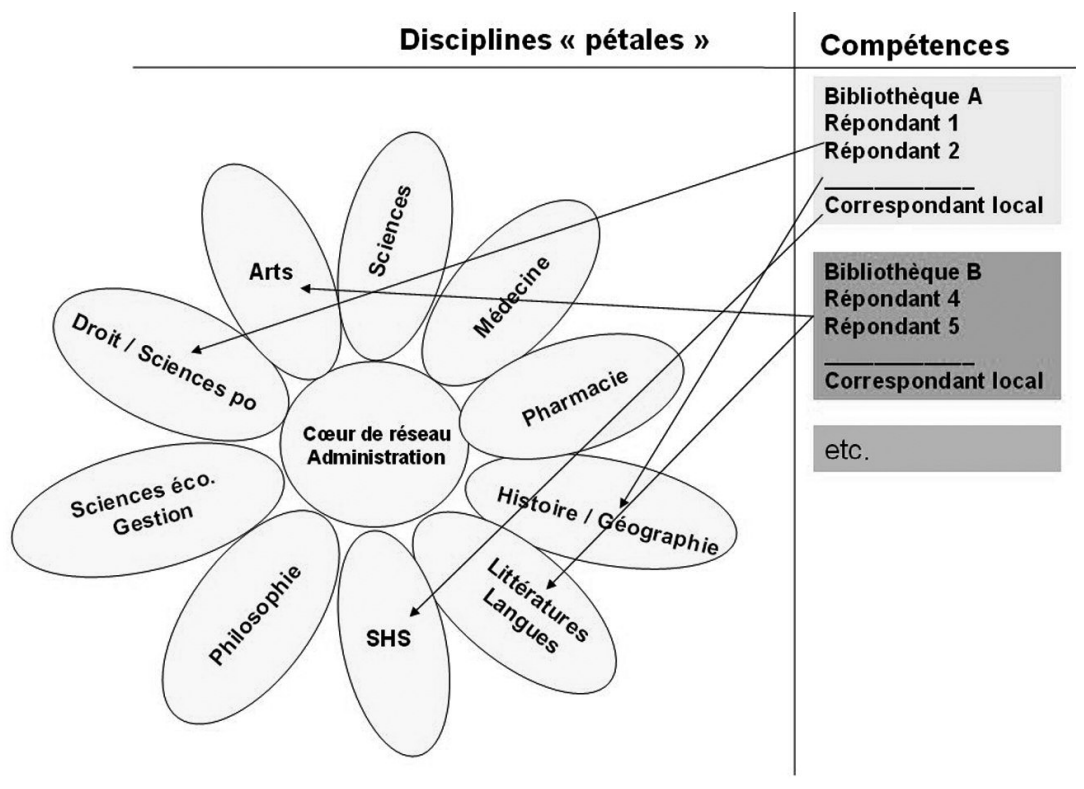

Pour la gestion de ce service, deux fonctions ont été créées :

- la fonction d'administrateur de secteur disciplinaire. Responsable du suivi scientifique d'une discipline, l'administrateur assure le contrôle-qualité des réponses, leur homogénéité dans le respect de la charte-qualité. Il assure aussi l'animation de l'équipe de répondants de sa discipline ;

- la fonction de coordinateur, rôle plus administratif d'interface entre Rue des Facs et un établissement physique dont il est le correspondant au sein du service virtuel. 


\section{PARTENARIAT ET MISE EN CEUVRE}

En 2006, le projet de référence virtuel se voit offrir le soutien de l'Université Numérique en Région (UNR) Paris Île-de-France. Il est officiellement porté par le groupe « Services documentaires » de l'UNR.

Ce nouveau statut est synonyme de nouvelle opportunité. Ainsi labellisé, le projet de service de référence virtuel devint éligible à un financement du département de Paris ${ }^{2}$, engagé dans l'offre de services numériques pour les étudiants parisiens. Ce financement a permis au dispositif de se doter d'un chef de projet, d'un abonnement à une plateforme de questions-réponses (QuestionPoint/OCLC) et de lancer une campagne de communication.

Durant cette phase de maturation et de finalisation du projet, de nombreuses bibliothèques se sont interrogées sur les modalités à mettre en œuvre pour proposer un service efficace et sur les moyens mobilisables au sein de chaque établissement. Le périmètre des bibliothèques participantes s'est dans le même temps élargi et le choix de proposer le service de discussion en direct (chat) en parallèle aux réponses différées a été arrêté. L'organisation thématique choisie dès 2005 et la création des bibliothèques virtuelles regroupant tous les répondants d'une même discipline se sont révélées l'un des points forts du dispositif. Il permet en effet l'intégration d'un établissement proposant des disciplines déjà couvertes par Rue des Facs avec une grande souplesse : les nouveaux répondants, sitôt formés, rejoignent une équipe opérationnelle sur laquelle ils peuvent s'appuyer pour faire leurs premières armes. La participation des nouveaux établissements permet de plus une amélioration de la qualité du service (délais de réponse plus courts, élargissement des plages de chat).

Symétriquement, seul un nombre restreint de volontaires est nécessaire pour ouvrir une nouvelle discipline. L'intégration progressive de nouveaux établissements a ainsi été nécessaire pour créer de nouveaux secteurs disciplinaires (Droit et sciences politiques, Sciences). 
De janvier 2009, date d'ouverture du service au public à février 2010, plus de 1700 questions ont été traitées. Compte tenu de la fermeture du service pendant l'été (du 14 juillet au 31 août), ce nombre de questions traitées dénote une activité importante et atteste d'un succès très certain auprès des usagers.

Rue des Facs couvre désormais tous les champs du savoir. Il s'agit bien là d'un véritable service interétablissement de coopération documentaire sur le mode virtuel, qui vit et s'agrandit en permanence grâce aux compétences mutualisées de plus de 250 bibliothécaires répondants et à une organisation du travail garantissant la liberté de chaque établissement participant. 


\section{4}

LE SI@DE, UN LABEL POUR UN RÉSEAU DE COOPÉRATION ENTRE SERVICES DE QUESTIONS-RÉPONSES

Le Si@de < http://www.bnf.fr/fr/collections_et_services/poser_une_ question_a_bibliothecaire/s.charte_siade.html >

Le Si@de s'est mis en place au $2^{\mathrm{e}}$ trimestre 2009. Il compte 15 adhérents : le réseau Bibliosésame et 14 établissements dont les services fonctionnent de manière autonome : les bibliothèques nationales suisse et du Québec, des bibliothèques universitaires, municipales, ou spécialisées. On peut citer parmi elles la Bibliothèque municipale de Lyon, l'enssib, la Cité de la santé. Parmi les domaines d'excellence, on trouve la médecine, la Chine du $\mathrm{xx}^{\mathrm{e}}$ siècle, la littérature pour la jeunesse, l'art contemporain, l'histoire du livre.

\section{LE SI@DE : SERVICES D'INFORMATION À LA DEMANDE}

Le Si@de est la mise en réseau de services existants, différent donc de Rue des Facs ou d'Ubib.fr. Cette mise en réseau s'est effectuée selon deux axes : une mutualisation des compétences et des ressources, et un engagement dans une politique de service commune.

\section{MUTUALISATION DES COMPÉTENCES}

Chaque établissement a défini pour son service ses domaines d'excellence dans le Si@de ; ceux-ci peuvent être plus restreints que l'ensemble des domaines dans lesquels il répond à ses usagers. Les services peuvent se transmettre les questions qu'ils reçoivent selon ces domaines d'excellence : ils savent que c'est la bibliothèque la mieux à même de répondre dans ce domaine qui traitera la question. Un service peut également transmettre une question parce qu'il est trop chargé au moment où il la reçoit, et qu'il sait que telle bibliothèque est compétente dans le domaine. 


\section{UNE POLITIQUE COMMUNE DU SERVICE RENDU}

Le Si@de s'est doté d'une charte qui définit les grands principes du service rendu à l'utilisateur : délai de réponse, type de questions auxquelles on répond, contenu de la réponse... Les adhérents au Si@de reçoivent un label ; celui-ci garantit à la bibliothèque qui transmet une question que celle-ci sera traitée selon les standards de la charte.

\section{EN PRATIQUE}

Chaque bibliothèque indique sa participation au Si@de sur la page d'accueil de son service questions-réponses par un lien hypertexte. Celui-ci permet à l'internaute de consulter la liste des bibliothèques adhérentes et leurs domaines d'excellence, ainsi que la charte qu'elles s'engagent à observer.

\section{POURQUOI UN RÉSEAU DE CE TYPE ?}

\section{LE CONTEXTE}

Au début de l'année 2006, quand commence la réflexion à la BnF, il existe déjà des services de questions-réponses : en France, les RADis à la Bpi, Le Guichet du Savoir à la Bibliothèque municipale de Lyon ; dans les pays francophones, Swissinfodesk à la Bibliothèque nationale suisse et le service de la Bibliothèque et Archives nationales du Québec. SINDBAD, le service de la $\mathrm{BnF}$, vient d'être lancé. La Bpi met en place le réseau Bibliosésame en janvier 2006. La BnF souhaite s'associer à ces services dans le cadre d'une structure souple, ouverte aux futurs services qui vont se mettre en place. À la BnF et dans les autres bibliothèques, les services sont généralistes ; il n'existe pas entre eux de complémentarité évidente sur un plan thématique. Ils ont par ailleurs chacun une identité forte et des organisations internes spécifiques.

\section{COMMENT TRAVAILLER ENSEMBLE ?}

L’idée directrice sera de définir un terrain commun sur lequel tous puissent s'entendre et s'appuyer pour coopérer, tout en permettant à chaque service de garder son autonomie, notamment son nom et son fonctionnement interne. Le terrain commun sera défini par les grands principes de 
service de la charte et se concrétisera par le label. La coopération devait se concrétiser dans l'échange des questions entre adhérents. S'est alors posée la question d'un outil commun : il présenterait l'avantage de faciliter les échanges, la production de statistiques, de permettre si souhaité la constitution d'une base de connaissances commune. Cependant, en dehors de la BnF, de la Bpi et de l'enssib, les bibliothèques intéressées par la coopération étaient déjà équipées de logiciels différents. Par ailleurs, imposer un logiciel aux futurs adhérents risquait de fermer la porte à certains établissements. Le courriel s'est avéré un bon moyen d'échanger les questions pour les bibliothèques utilisant des logiciels différents. Il a donc été décidé de ne pas imposer de logiciel commun.

\section{UN PREMIER BILAN}

Il est un peu tôt, à la date de la rédaction de cet ouvrage, pour faire un bilan sur la réorientation de questions. Des échanges de questions réguliers se mettent en place entre certains services. Les domaines de compétences se combinent avec l'utilisation ou non d'un outil commun (ce que permet QuestionPoint par exemple) pour établir des partenariats plus privilégiés : pour la BnF par exemple, les échanges se font surtout avec la Bpi et avec l'enssib. La réorientation de la question permet à l'usager de voir sa question traitée par le service le plus compétent dans le domaine. Du côté des services, ces échanges de questions sont aussi l'occasion d'observer les pratiques de leurs partenaires pour réfléchir aux leurs. Par exemple, une question reçue à la $\mathrm{BnF}$ et considérée comme une demande de consultation médicale, donc hors du champ du service, a été traitée par la Cité de la santé, qui lui a apporté une réponse d'un point de vue documentaire, en invitant l'usager à se rapprocher de son médecin pour une réponse de praticien.

L'avenir du Si@de ? Un accord a été conclu entre les partenaires jusqu'en octobre 2011. Au vu du bilan, les établissements fondateurs décideront alors de la suite à donner à cette expérience. 


\section{PARTIE IV}

\section{ADAPTER SON SERVICE À L'ENVIRONNEMENT ET À LA NATURE DE SES USAGERS ET DE SES COLLECTIONS}

1. TYPOLOGIE DES SERVICES DE QUESTIONS-RÉPONSES par Claire Nguyen

2. CONSTRUIRE UN SERVICE DE RÉFÉRENCE GÉNÉRALISTE : L'EXEMPLE DU GUICHET DU SAVOIR DE LA BIBLIOTHÈQUE MUNICIPALE DE LYON par Christelle Di Pietro

3. SINDBAD, LES PUBLICS ET LES COLLECTIONS DE LA BIBLIOTHĖQUE NATIONALE DE FRANCE par Isabelle Copin

4. DE BIBLIOSÉS@ME À BIBLIOSÉSAME.ORG : UN RÉSEAU GÉNÉRALISTE DE QUESTIONS-RÉPONSES DE BIBLIOTHĖQUES PUBLIQUES, PREMIER BILAN par Nathalie Daigne

5. VOUS AVEZ DES QUESTIONS SUR BIBLIOSÉSAME ? LES BIBLIOTHÉCAIRES DU RÉSEAU VOUS RÉPONDENT ! L'EXEMPLE DE LA BMVR DE MARSEILLE par Annie Prunet

6. UN SERVICE DE QUESTIONS-RÉPONSES EN LIGNE DE BIBLIOTHĖQUE SPÉCIALISÉE : BIUMINFO, LE SERVICE DE LA BIBLIOTHĖQUE INTERUNIVERSITAIRE DE MÉDECINE ET D'ODONTOLOGIE par Hélène Gautier-Gentès

7. QUESTIONS-SANTÉ, LE SERVICE DE RÉPONSES EN LIGNE DE LA CITÉ DE LA SANTÉ par Sandrine Lebastard et Tù-Tâm Nguyên

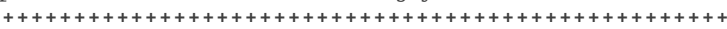
8. LE SERVICE QUESTIONS ? RÉPONSES ! DE L'ENSSIB par Pierre Moison 


\section{1}

\section{TYPOLOGIE DES SERVICES par Claire Nguyen DE QUESTIONS-RÉPONSES EN LIGNE}

Il n’y a pas une mais des typologies des services de questions-réponses. Je propose donc ces typologies selon le producteur du service (relevant de services documentaires ou non documentaires).

Je reviendrai après sur les services documentaires, en les classant selon :

- la nature, généraliste ou spécialisée, du service ;

- le mode et les techniques employés pour répondre à la question ;

- l'ouverture du service : restreint/ouvert ; gratuit/payant. Je ferai ensuite une présentation des différents types de service collaboratifs.

\section{LES SERVICES DE Q/R SELON LE PRODUCTEUR DU SERVICE}

- Les services documentaires, émanant de bibliothèques ou d'institutions publiques.

- Les services non documentaires. Il peut s'agir de services de Q/R émanant d'autres institutions publiques, de « guichets » virtuels (impôts, mairies...).

Depuis une quinzaine d'années, des sites qu'on appelle Q\&A (Questions and Answers) fleurissent, disparaissent ou reviennent sur le Net. Généralistes (Askville d'Amazon, Aardvark ${ }^{1}$, Mahanswerbag, Wikianswers, Blurtit.com) ou plus spécialisés (comme le site scientifique < scientificamerican.com >). Ils sont très souvent gratuits comme Yahoo Answers ${ }^{2}$, reposant sur un réseau de répondants bénévoles, dont les réponses sont évaluées par les inter-

1. Cf . l'article de N. Alarcon, « Comment promouvoir un service de renseignement en ligne ?», p. 51. 
nautes. Il existe quelques sites payants généralistes ou grand public ; la rétribution étant modeste, parfois à la discrétion du questionneur (Justanswers - qui répond beaucoup à des questions de santé), avec des experts parfois sélectionnés, les « AskA » (Ask An Expert) ${ }^{3}$. Il existe toujours les services SVP orientés vers les entreprises et les professionnels, qui sont passés du téléphone au virtuel ${ }^{4}$. Des bibliothécaires se sont lancés à l'assaut de ce "marché » très compétitif des AskA comme IPL2 ${ }^{5}$ aux ÉtatsUnis qui s'appuie sur des étudiants et professionnels en sciences de l'information bénévoles.

Ce paysage est marqué par une forte concurrence; certains grands sites ont fermé (Webhelp ; Google answers).

On ne dénombre plus les légions de forums de sites généralistes ou grand public comme les forums médicaux ou juridiques, qui peuvent être modérés par un expert dans le domaine (en droit net-iris ${ }^{6}$; en médecine : Santé Canoë au Québec ${ }^{7}$ ).

\section{SERVICES SYNCHRONES, SERVICES ASYNCHRONES 8}

3. Voir liste des Aska sur : < http://www.refdesk.com > (consulté le 6 mai 2010).

4. < http://www.svp.com/fr/ > (consulté le 6 mai 2010).

5. IPL2 : <http://www.ipl.org> (consulté le 3 mai 2010).

6. < http://www.net-iris.fr > (consulté le 2 juin 2010).

7. < http://sante.canoe.com/ask_an_expert_general.asp > (consulté le 6 mai 2010).

8. Il s'agit d'un tableau des principales caractéristiques, atouts ou défauts de ces modes. Je ne reviendrai pas sur le détail de ces services, bien développés dans les articles d'H. Tardif, «Quels outils pour quels services ? Quelle technologie choisir ? », p. 94 et de J.-C. Houpier, « Service de questions-réponses et environnement de l'usager : quelle intégration ? », p. 59. 


\section{TYPOLOGIE DES SERVICES DE Q/R SYNCHRONES}

\section{TABLEAU 1 ET ASYNCHRONES}

\begin{tabular}{|c|c|c|c|c|c|}
\hline \multicolumn{3}{|c|}{$\begin{array}{l}\text { Mode asynchrone (ou en différé) } \\
\text { + Temps de faire une réponse construite } \\
\text { - Décalage et délai entre la question } \\
\text { et la réponse }\end{array}$} & \multicolumn{3}{|c|}{$\begin{array}{l}\text { Mode synchrone (ou en temps réel) } \\
\text { + Interactivité } \\
\text { - Requiert forces humaines, bonne } \\
\text { organisation et formation spécifique }\end{array}$} \\
\hline outil & Avantages & $\begin{array}{l}\text { Inconvénients/ } \\
\text { point de vigilance }\end{array}$ & outil & Avantages & Inconvénients \\
\hline Courriel & $\begin{array}{l}1^{\text {er }} \text { degré des } \\
\text { services de } Q / R \text { : } \\
\text { extrême facilité } \\
\text { d'installation }\end{array}$ & $\begin{array}{l}\text { Manque } \\
\text { d'interactivité ; } \\
\text { temps différés } \\
\text { Veiller à la } \\
\text { traçabilité et à } \\
\text { l'archivage des Q/R }\end{array}$ & $\begin{array}{l}\text { Messag } \\
\text { erie } \\
\text { instan- } \\
\text { tanée }\end{array}$ & $\begin{array}{l}\text { Gratuité } \\
\text { Environnement } \\
\text { des jeunes } \\
\text { usagers }\end{array}$ & $\begin{array}{l}\text { Pas de } \\
\text { possibilité de } \\
\text { comptes } \\
\text { partagés entre } \\
\text { bibliothécaires }\end{array}$ \\
\hline Formulaire & $\begin{array}{l}\text { Facilité } \\
\text { d'installation } \\
\text { Possibilité de } \\
\text { cerner la ques- } \\
\text { tion et le profil } \\
\text { de l'usager }\end{array}$ & $\begin{array}{l}\text { Manque } \\
\text { d’interactivité ; } \\
\text { temps décalés }\end{array}$ & \multirow[t]{5}{*}{$\begin{array}{l}\text { Chat, } \\
\text { intégré } \\
\text { dans } \\
\text { une } \\
\text { plate- } \\
\text { forme } \\
\text { Web }\end{array}$} & \multirow{5}{*}{$\begin{array}{l}\text { Programmes } \\
\text { évolués et, } \\
\text { plus complexes, } \\
\text { combinant } \\
\text { différents outils } \\
\text { (courriel, chat, } \\
\text { formulaire) } \\
\text { Fonctionnalités } \\
\text { de gestion, de } \\
\text { navigation et } \\
\text { de conversation } \\
\text { avancées }\end{array}$} & \multirow{5}{*}{$\begin{array}{l}\text { Souvent } \\
\text { propriétaires, } \\
\text { ces outils } \\
\text { représentent } \\
\text { un certain } \\
\text { coût } \\
\text { Maintenance } \\
\text { et relations } \\
\text { suivies avec le } \\
\text { prestataire }\end{array}$} \\
\hline Forum & $\begin{array}{l}\text { Proche des habi- } \\
\text { tudes inter- } \\
\text { nautes } \\
\text { Publication, } \\
\text { valorisation des } \\
\text { réponses des } \\
\text { bibliothécaires }\end{array}$ & $\begin{array}{l}\text { Pas de } \\
\text { confidentialité }\end{array}$ & & & \\
\hline Blog & $\begin{array}{l}\text { Toutes les } \\
\text { fonctionnalités } \\
\text { d'un outil Web } \\
\text { 2.0. proche des } \\
\text { habitudes des } \\
\text { internautes }\end{array}$ & $\begin{array}{l}\text { Pas de recherche } \\
\text { avancée ni de tris } \\
\text { des recherches } \\
\text { Pas de publication } \\
\text { de question en } \\
\text { direct }\end{array}$ & & & \\
\hline $\begin{array}{l}\text { Réseaux } \\
\text { sociaux, } \\
\text { monde } \\
\text { virtuel }\end{array}$ & $\begin{array}{l}\text { Insertion dans } \\
\text { l'environnement } \\
\text { de l'usager } \\
\text { Extrême mobilité }\end{array}$ & $\begin{array}{l}\text { Peu de fréquenta- } \\
\text { tion pour le } \\
\text { moment }\end{array}$ & & & \\
\hline Sms & Outil d'avenir ${ }^{9}$ & $\begin{array}{l}\text { Ne convient pas à } \\
\text { toutes les questions } \\
\text { (bien pour ques- } \\
\text { tions factuelles, } \\
\text { pratiques). }\end{array}$ & & & \\
\hline
\end{tabular}




\section{SERVICE SPÉCIALISÉ OU GÉNÉRALISTE}

Un service généraliste répondra à des questions de toute discipline dans tous les domaines du savoir. Ce sont plutôt les bibliothèques publiques qui offrent ce type de service même si elles peuvent aussi vouloir valoriser des thématiques particulières, régionales par exemple (BiblioSésame, SINDBAD). Les bibliothèques universitaires à large spectre disciplinaire proposent donc des services pluridisciplinaires.

Un service de Q/R spécialisé est le reflet de la spécificité, de la spécialité de la bibliothèque. C'est le cas de BIUMINFO de la BIUM, qui répond à des questions de santé, ou Questions-santé (Cité de la Santé). La spécialisation peut être aussi issue d'un choix de la politique de service : la BN Suisse, qui offre des collections dans tous les domaines du savoir, ne répond qu'aux questions sur la Suisse ${ }^{10}$. Ou il peut l'être par la nature du public-cible : le réseau britannico-australien, Chasing the Sun ne répond qu'aux questions de professionnels de santé.

\section{TYPES DE SERVICE ET ACCESSIBILITÉ}

Un service de $Q / R$ en ligne peut être défini aussi selon son accessibilité. Il peut être ouvert à tous ou réservé à une communauté définie (communauté universitaire, inscrits d'une bibliothèque publique). Le filtrage est susceptible de s'effectuer via un code postal, barrière facilement contournable, un numéro de lecteur, un accès authentifié à l'ENT.

Il peut être gratuit ou payant. Certains services proposent leurs services aux entreprises contre rétribution (Toulouse 1) ; les recherches deviennent payantes chez SwissInfoDesk à partir de 30 minutes (seuil au-delà duquel la recherche est considérée comme complexe).

9. En France, il n'est utilisé que pour les alertes (réservations, amendes..). Il est promis à un bel avenir cependant pour la référence car selon la dernière enquête de la SOFRES de décembre 2009, 79 \% des Français en sont équipés (la tranche la plus équipée étant les 18/24 ans). Il est utilisé comme outil de renseignement en ligne par nos collègues américains (par exemple, l’Université de Yale).

10. SwissInfoDesk : < http://www.nb.admin.ch/dienstleistungen/swissinfodesk/index.html? > (consulté le 6 mai 2010). 
Les échanges peuvent être publiés ou confidentiels. Pour les bibliothèques académiques, on peut comprendre que des chercheurs préfèrent garder leur questionnement, et leurs sujets de recherche, confidentiels. En plus des forums (comme Le Guichet du Savoir), publics par nature, les services qui publient les questions et réponses demandent aux utilisateurs leur autorisation - qu'il s'agisse de toutes les questions (comme le service de l'enssib) ou d'une sélection (BiblioSésame, SINDBAD). La publication se fait après anonymisation des questions.

\section{LES RÉSEAUX COLLABORATIFS}

Les services locaux de Q/R sont créés, maintenus, développés, assurés par un établissement donné ; a contrario, les services collaboratifs, qu'on appelle les réseaux de (questions)-réponses regroupent des bibliothèques qui mettent leurs ressources en commun, selon des modalités très variables, pour répondre aux usagers ${ }^{11}$.

Le tableau de la page suivante propose une typologie " politique » des réseaux, qui utilisent en grande majorité des plateformes Web, développées pour la collaboration.

Un service peut être basé sur des critères identitaires spécifiques, sur des critères locaux, ou les deux ou mixtes.

Cette mixité pose également la question des périmètres : tout réseau est susceptible de s'agrandir, d'évoluer, de changer de modèle et d'échelle... ou de se mettre en relation avec d'autres réseaux dans un maillage assez serré pour répondre et satisfaire un maximum d'usagers-internautes. De plus, il devient intéressant d'appartenir à plusieurs réseaux, comme c'est le cas par exemple pour la Bibliothèque Sainte-Geneviève (Rue des Facs, Si@de).

11. Cf. le Mode d'emploi, p. 9 et l'article de C. Bruley, « Le renseignement en ligne au SCD Lyon 1: la démarche projet », p. 47.

12. Site de QuestionPoint : < http://www.questionpoint.org > (consulté le 6 mai 2010). 


\section{TABLEAU 2 LES SERVICES COLLABORATIFS}

\begin{tabular}{l|l|l}
\hline $\begin{array}{l}\text { Modèle } \\
\text { de réseau }\end{array}$ & critère / service & Exemples \\
\hline
\end{tabular}

Services basés sur leur identité ou sur la spécificité de leurs publics, de leurs missions

\begin{tabular}{|l|l|l|}
$\begin{array}{l}\text { Service } \\
\text { spécifique }\end{array}$ & $\begin{array}{l}\text { - disciplinaire ou thématique } \\
\text { - identifié par une thématique ou } \\
\text { une discipline }\end{array}$ & $\begin{array}{l}\text { Collaborative Virtual Reference } \\
\text { Service for Korean Studies ; } \\
\text { Library Lawline (droit) }\end{array}$ \\
\hline $\begin{array}{l}\text { - institutionnel } \\
\text { - relevant d’institutions similaires ou } \\
\text { ayant des missions similaires }\end{array}$ & $\begin{array}{l}\text { BiblioSésame (bibliothèques } \\
\text { publiques) ; } \\
\text { Rue des Facs ; Pregunte (biblio- } \\
\text { thèques publiques espagnoles) }\end{array}$ \\
\hline $\begin{array}{l}\text { - associatif } \\
\text { - faisant partie d'une association déjà } \\
\text { unstituée de bibliothèques ayant }\end{array}$ & Si@de \\
\hline - de public & défini par le public desservi & $\begin{array}{l}\text { Infoeyes (handicapés, USA) } \\
\text { Chasing the sun (professionnels } \\
\text { de santé, Australie et Grande- } \\
\text { Bretagne) }\end{array}$ \\
\hline
\end{tabular}

Services basés sur une proximité géographique, pouvant mêler des bibliothèques publiques, académiques, hospitalières, de musées, de centres de documentation, etc.

\begin{tabular}{|c|c|c|}
\hline \multirow[t]{4}{*}{$\begin{array}{l}\text { Service } \\
\text { géo- } \\
\text { localisé }\end{array}$} & $\begin{array}{l}\text { - régional } \\
\text { - à l'échelle locale : ville, région, } \\
\text { regroupement de régions }\end{array}$ & $\begin{array}{l}\text { - à l'échelle d'une ville : Rue des } \\
\text { Facs (à ses débuts); } \\
\text { - d'une région : AskUs (Maryland, } \\
\text { USA), OhioLink (Ohio, USA), } \\
\text { AskScotland (Ecosse), Ubib.fr }\end{array}$ \\
\hline & $\begin{array}{l}\text { - national } \\
\text { - desservant tout un pays }\end{array}$ & $\begin{array}{l}\text { Deutsche Internet Bibliothek } \\
\text { (Allemagne) ; Biblioteksvar } \\
\text { (Norvège) ; } \\
\text { Pregunte (Espagne) }\end{array}$ \\
\hline & $\begin{array}{l}\text { - transnational } \\
\text { - à cheval sur plusieurs pays. Parfois } \\
\text { basés sur une communauté de langue }\end{array}$ & Chasing the sun \\
\hline & $\begin{array}{l}\text { - mondial } \\
\text { - sans frontières. Une question peut être } \\
\text { (ré-)orientée vers toute bibliothèque, } \\
\text { tout type, tout public }\end{array}$ & Réseau de QuestionPoint ${ }^{12}$ \\
\hline
\end{tabular}


CONSTRUIRE UN SERVICE DE RÉFÉRENCE GÉNÉRALISTE : L'EXEMPLE DU GUICHET DU SAVOIR DE LA BIBLIOTHĖQUE MUNICIPALE DE LYON

\section{2}

par Christelle

Di Pietro

Le Guichet du Savoir < http://www.guichetdusavoir.org >

\section{POURQUOI LE GUICHET ? UN CONTEXTE PARTICULIER}

L'idée d'un service de questions-réponses en ligne germe en 2002 au sein d'un groupe de travail transversal à la bibliothèque sur les nouveaux services. Le principe de départ est simple : reprendre le concept de SVP, célèbre service de question réponse initié en 1935 (!) par téléphone, et de proposer un service qui aurait réponse à tout et à tous. Transposer le service sur Internet a semblé évident : la BmL est l'une des premières bibliothèques françaises à avoir investi le Web, dès 1996, un an après la départementalisation de la bibliothèque de la Part-Dieu qui a elle aussi été l'une des causes, plus lointaine, de la création du Guichet.

La départementalisation de la bibliothèque de la Part-Dieu a influencé très positivement la fréquentation et l'usage des collections : elle s'est traduite en cinq ans par un doublement des prêts, mais n'a pas suffi à enrayer un mouvement de fond qui s'accélère depuis le milieu des années 2000. Alors que la fréquentation était plutôt en augmentation jusqu'en 2003, elle commence à chuter, puis à stagner : en 2004, on comptait 1078138 entrées (bibliothèque de la Part-Dieu seulement), mais 972751 en 2008.

Cette stagnation de la fréquentation s'est accompagnée de celle de l'usage documentaire (notamment du prêt), avec un net déplacement vers les supports " non imprimés ", cd-rom d'abord, vite balayés par les cd et dvd et bien sûr l'offre en ligne, comme le montrait Bertrand Calenge en 2003. Ces publics qui désertent et manquent cruellement dans les rangs de la bibliothèque (à Lyon ou ailleurs) correspondent à peu près à la population des actifs qui ne dépasse guère les $20 \%$ d'inscrits (alors qu'ils représentent $60 \%$ de la population lyonnaise). 
On peut cependant supposer que ce public a des besoins, sinon documentaires, du moins d'information : vie quotidienne, travail, curiosité, nombreuses sont les occasions qui exigent d'obtenir des renseignements précis et fiables. Or, l'offre traditionnelle de bibliothèque, sous forme de stock offert à qui peut se déplacer, est inadaptée aux contraintes du quotidien de la majorité de la population, et pas seulement pour des raisons liées aux horaires ou aux déplacements.

À qui s'adresser lorsqu'on souhaite non pas emprunter un livre dont on connaît l'existence, mais une simple information factuelle, et que l'on ne peut pas mobiliser de compétences propres pour la trouver?

Un autre impact décisif de la départementalisation a été l'acquisition de compétences de contenus par les bibliothécaires. La spécialisation thématique a obligé les acquéreurs des départements à s'approprier les connaissances propres à leurs domaines. Se sont ainsi constituées au fil du temps des équipes spécialisées, d'autant plus concentrées sur l'activité liée au contenu qu'elles ont été soulagées des opérations de transaction grâce à la création d'un service de prêt centralisé.

L'inscription dans un projet en adéquation avec la politique de la Ville a emporté la concrétisation du service. La Ville de Lyon s'est fortement inscrite dans le développement du numérique et des nouveaux services, elle s'est dotée d'une "pépinière numérique », le Plan lyonnais pour la société de l'information. Ce service a financé et promu le projet (3 postes à temps complet ont été créés). Desservir des usagers non-lyonnais sur des subsides locaux n'a pas posé question dans ce contexte : Lyon a fait du numérique l'un de ses axes majeurs de développement.

Une idée, des compétences, des besoins, un support politique et financier : Le Guichet du Savoir est acté à l'automne 2003, le chantier du projet démarre en janvier suivant, le service s'ouvre le 29 avril 2004.

\section{LA DÉFINITION DES MISSIONS}

Dès la prise de décision de création du service, un comité de pilotage interne a été constitué avec le directeur des bibliothèques de Lyon, une chef de projet, le responsable de la communication interne et de l'évaluation, le responsable informatique et le coordinateur du Pôle Part-Dieu. Ce comité 
a eu en charge la validation de toutes les modalités de fonctionnement du service avant sa mise en œuvre, à commencer par la définition des missions et l'objectif du Guichet du Savoir (GdS).

Les missions du service sont a priori très largement ouvertes : tout type de questions sur tous les sujets sont acceptés. Recherches bibliographiques et documentaires, informations factuelles, locales, pratiques, méthodologie de recherche, découvertes d'outils : un seul mot d'ordre : répondre à la demande. Ce point n'a pas réellement fait l'objet d'un débat avant l'ouverture : la BmL est une bibliothèque encyclopédique, il semble logique de tout traiter. A l'épreuve de la réalité des questions reçues, il a fallu à certaines occasions recadrer, non pas le champ des questions mais le cadre de la réponse, et définir des limites, notamment en regard de la compétence du questionneur (étudiants par exemple). L'exercice le plus délicat du répondant n'est quelquefois pas de trouver une réponse mais d'en déterminer la profondeur : quel niveau de spécialisation pour quel questionneur?

L'un des partis pris de départ les plus importants a été l'anonymat des usagers du service. Si nous avons envisagé au moment de l'élaboration des modalités de fonctionnement de restreindre le service (aux seuls inscrits de la $\mathrm{BmL}$ ou par localisation géographique), ou bien encore de demander des éléments d'identification via un formulaire, nous y avons renoncé assez vite. En effet, dans la mesure où nous souhaitions nous adresser au public des absents, public par définition non ou peu familier de l'usage bibliothèque, il fallait ne pas mettre de barrière: pas de pré-requis culturel (demander par exemple le niveau de diplôme de la personne peut être négativement ressenti), pas de formalité, totale gratuité. Limiter le service à une zone géographique présente une incongruité pour un service Web et des développements techniques complexes, avec un risque de se heurter à un réel problème de droit (au moins communautaire) quant à l'égalité de l'accès aux services publics ${ }^{1}$.

1. Une jurisprudence européenne de 1999 a rappelé que la différenciation de l'accès aux services publics accordée par des communes sur la base de la résidence et de la non-résidence n'est pas conforme au droit communautaire (Arrêt du 29 avril 1999 - Ciola). Lire l'article « Les tarifs préférentiels accordés aux résidents par les services publics locaux sont-ils conformes au droit communautaire ? " $1^{\text {er }}$ février 2007. [En ligne] < http://carrefourlocal.senat.fr/vie_locale/cas_pratiques/tarifs_preferentiels_accordes/ index.html > (consulté le 6 mai 2010). 


\section{DEUX PROBLÉMATIQUES LANCINANTES : LÉGITIMITÉ ET AUTONOMIE}

Deux questions importantes ont dû être réglées en préambule à la réflexion sur le fonctionnement du service : celle de la légitimité des questions (et des questionneurs), qui dérive immanquablement vers celle de "l'autonomie de l'usager » et celle de la transversalité de service dans la bibliothèque qui a finalement abouti au choix d'une équipe centralisée s'appuyant sur des équipes spécialisées.

Sans s'appesantir sur la question de l'autonomie de l'usager qui a déjà été largement évoquée - la plus complète des synthèses sur ce sujet étant celle de Bertrand Calenge sur son blog ${ }^{2}$-, voici quelques éléments de débat. La prise en charge de la formation des usagers en bibliothèque municipale concerne initialement l'apprentissage de l'utilisation du lieu « bibliothèque » : se repérer dans les rayonnages, utiliser l'OPAC, comprendre les bases du classement (quoique cette conception soit largement dépassée par les pratiques induites par l'Internet), mais n'a jamais concerné l'apprentissage de techniques de recherche documentaire complexes. Par un glissement sémantique étrange, certains bibliothécaires en sont arrivés à considérer l'autonomisation des usagers comme une formation à la recherche documentaire de façon à ce qu'ils se débrouillent seuls. Cette posture est non seulement absurde, mais dangereuse pour le devenir des professionnels des sciences de l'information : elle méconnaît d'une part la réelle envie des usagers à vouloir être formés, et elle réduit d'autre part la compétence du chercheur d'information à de simples équations de recherche que l'on saisirait dans une base de données, alors qu'il s'agit d'une réelle compétence professionnelle à forte valeur ajoutée, nécessitant une formation initiale solide et une longue expérience. Quant au désir supposé des usagers d'être formés, on peut certainement en mesurer l'inexistence à l'aune de l'abandon des bibliothèques par ceux qui veulent seulement trouver et pas chercher, abandon inversement proportionnel à l'ampleur du geste du bibliothécaire qui, derrière sa banque d'accueil, désigne vaguement le coin

2. Bertrand Calenge. À propos de l'autonomie de l'usager, in Carnet de notes, 22 janvier 2009. [En ligne] $<$ http://bccn.wordpress.com/2009/01/22/a-propos-de-lautonomie-de-lusager/ > 
de rayonnage dans lequel la personne qui souhaite un renseignement trouvera son bonheur.

Il est impératif de régler cette fausse question de l'autonomie avant même que de pouvoir esquisser les contours d'un service de référence : si l'on estime que le rôle du bibliothécaire est de former son cher public à la complexité des outils et techniques documentaires comment accepter des questions appelant des réponses précises sans remettre en cause leur légitimité ? Le choix du Guichet a été celui d'une ouverture maximale : pas de question illégitime, mais pas non plus de leçon documentaire. Néanmoins, quiconque examinerait attentivement les réponses produites pourrait très bien y apprendre à pêcher...

\section{QUI RÉPOND ? QUELLE ORGANISATION DE SERVICE ?}

La question de la transversalité du service dans la bibliothèque relève à la fois de la stratégie de l'établissement en matière de gestion de ressources humaines et de l'organisation du travail. Derrière une question simple (le service est-il géré par une équipe unique dédiée ou bien repose-t-il sur l'ensemble des équipes de la bibliothèque ?), s'est jouée l'implication de l'ensemble des agents de la bibliothèque, non seulement dans le Guichet, mais dans le processus mis en œuvre par la BmL d'inscrire résolument les services de la bibliothèque dans la médiation de contenus documentaires ${ }^{3}$. C'est ainsi que le comité de pilotage du GdS a opté pour un fonctionnement mixte : une équipe centralisée et généraliste composée de 3 personnes -1 bibliothécaire et 2 assistantes qualifiées de conservation du patrimoine et des bibliothèques - qui s'appuie sur les départements spécialisés de la bibliothèque de la Part-Dieu. À ces départements centraux ont été joints les départements spécialisés des deux médiathèques d'arrondissements : Arts Vivants à la médiathèque de Vaise et Cap'Culture Santé à la médiathèque du Bachut.

Le service du GdS a pour mission de lire la totalité des questions, de les répartir sur les différents départements thématiques, de traiter une partie

3. En 2006, la BmL ouvre un nouveau service basé sur l'exploitation des compétences documentaires des bibliothécaires : Point d'Actu, d'autres services en ligne thématiques suivront : Cap'Culture Santé, le portail Arts Vivants, etc. Cf. l'article de B. Calenge, «Valoriser le service de questions-réponses et l'intégrer aux autres services de la bibliothèque », p. 68 . 
des questions (entre 50 et $60 \%$ ) et de relire la totalité des réponses. Ce rôle de coordination est à la fois indispensable pour assurer la cohérence de la production et la régulation des flux, et délicat en terme de négociation lorsqu'il est amené à invalider une réponse. Cette dernière fonction est intrinsèquement liée au caractère public du service. Tant que l'échange entre le bibliothécaire et l'usager demeure privé, le risque que les réponses faites soient sujettes à caution engage peu la bibliothèque et sa tutelle (quoiqu'en ces temps de judiciarisation il vaille mieux être prudent), mais une publication aux yeux de tous démultiplie la possibilité d'être pris en faute sur une réponse fausse. Il est apparu indispensable que chaque réponse, du moins dans les premiers temps d'ouverture du service, fasse l'objet d'une vérification systématique sur au moins deux points : la réponse répond-elle à la question posée (pour autant que la réponse existe) ? Les sources citées sont-elles fiables ? À l'affirmative, la réponse est validée. Cela ne signifie pas pour autant, et de façon un peu paradoxale, que la réponse est vraie. Le rôle du bibliothécaire est limité à la " simple » validation des sources : il ne peut pas réellement s'assurer que l'information délivrée par cette source est totalement véridique, mais cette validation suffit à se défendre contre d'éventuels désaccords.

\section{PUBLIER OU NE PAS PUBLIER ?}

Le dernier point essentiel, très novateur, des modalités de mise en œuvre du service a été la décision de publier : une publication totale des questions et des réponses en accès inconditionnel, libre et gratuit, 24 heures sur 24. Lors de sa création, Le GdS est le seul service de Q/R généraliste à publier l'intégralité des échanges : le service espagnol équivalent, Pregunte, ne publie qu'une sélection, et le SVP de la Cité de la musique est spécialisé ${ }^{5}$. La publication a indiscutablement assuré le succès du GdS : alors que d'autres services, même en coopération, plafonnent à 150 questions par mois, Le GdS, lors des trois premières années de fonctionnement, dépasse les 500 questions mensuelles (voir le graphique ci-après).

4. Pregunte, las bibliotecas responden : < http://www.pregunte.es > (consulté le 6 mai 2010).

5. SVP musique, Cité de la musique : < http://www.cite-musique.fr/francais/questions/questions.aspx > (consulté le 6 mai 2010). 
Il existe au moins deux avantages immédiats à la publication : cela permet à l'usager-internaute de situer immédiatement le genre de service rendu et le type de question qu'il peut poser, et oblige le bibliothécaire-répondant à une grande vigilance dans l'élaboration de sa réponse. Dans la mesure où le public ciblé est un public non-usager des bibliothèques, il convient d'effacer à la fois les contraintes bibliothéconomiques (se repérer dans l'espace, interroger le catalogue par exemple) en proposant une interface simple, mais aussi le cadre culturel préétabli comme le proposent souvent les bibliothèques (ou tel qu'il peut être fantasmé).

\section{ÉVOLUTION DU NOMBRE DE QUESTIONS POSÉES AU GRAPHIQUE GUICHET DU SAVOIR D'AVRIL 2004 À DÉCEMBRE 2008}

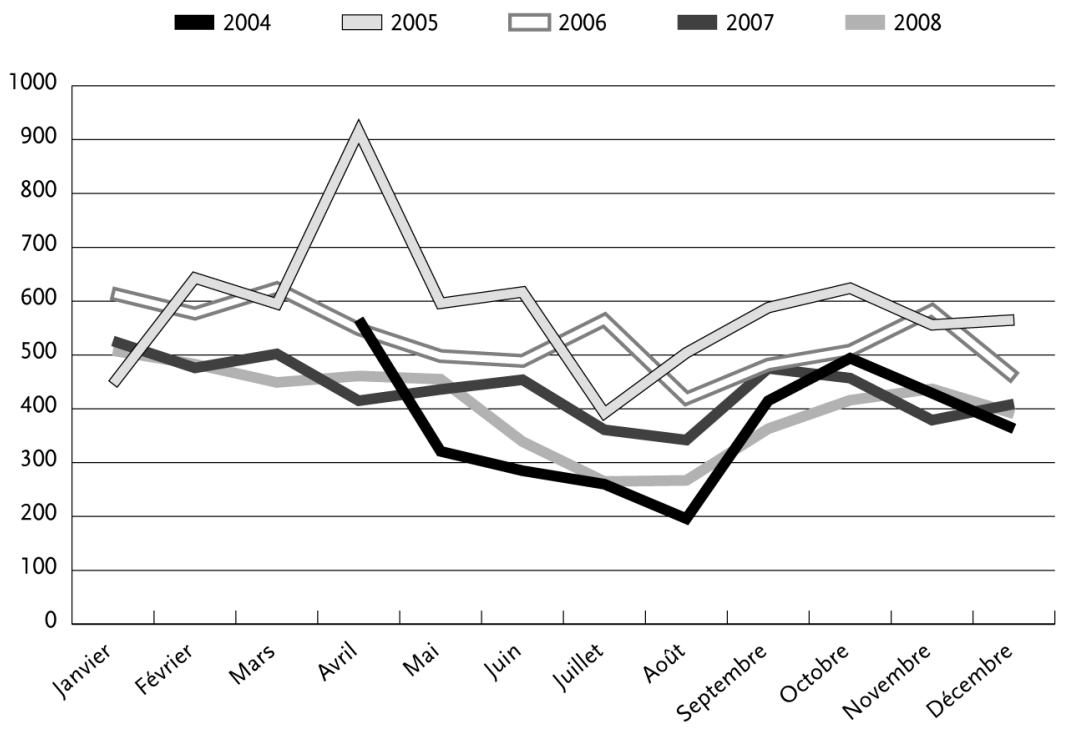

L'usager n'est pas tenu de se conformer à un protocole, ni même d'écrire sans fautes, tant que sa question est compréhensible. Et l'étendue des sujets abordés, visible dès l'entrée sur le site, permet de sortir du cadre stricte- 
ment culturel pour entrer dans celui de l'information au sens large : l'usager peut solliciter Le GdS sur n'importe quel thème (la vie domestique entre autres, ou les pratiques sexuelles par exemple).

Une autre conséquence est l'application stricte du respect du droit d'auteur qui n'est exercé que dans le cadre de l'exception de citation.

L'effet à moyen terme de la publication en ligne dans un format ouvert, (le forum du Guichet est en langage PHP), est une visibilité maximale sur le Web : des milliers de pages indexées ${ }^{6}$ et un public très vite internatio$\mathrm{nal}^{7}$. Le service assure ainsi sa propre promotion, avec peut-être à terme une interrogation sur la limite de cette publication. On peut constater depuis deux ans une baisse de l'activité, baisse inversement proportionnelle à l'augmentation du trafic Web $^{8}$ : peut-on supposer que compte tenu du nombre de demandes déjà traitées (presque 30000 ) et des consultations massives, les internautes trouvent leurs réponses sans avoir besoin de poser leur question?

\section{DES PRINCIPES CLAIRS ET INCHANGÉS, UNE ORGANISATION DU TRAVAIL OPÉRATIONNELLE}

\section{POSER DES PRINCIPES...}

Une fois les missions et le public définis, il était possible de formaliser des principes du service :

- toute question est légitime : le questionneur n'a pas à justifier d'un cadre particulier de recherche pour utiliser le service, sauf s'il le souhaite ou que cela est nécessaire pour éclairer sa question ;

- les formalités d'accès sont minimalisées : un pseudo, un mot de passe, une adresse e-mail valide et l'usager peut poser sa question ;

- les questions-réponses sont publiées ;

6. À l'heure de la rédaction de cet article, 17 mars 2010, 59800 pages du GdS sont indexées dans Google : < http://www.google.fr/search?q=site\%3Aguichetdusavoir.org > (consulté le 6 mai 2010).

7. Le Guichet a été consulté dans les cinq continents seulement trois semaines après son ouverture.

8. Le Guichet a franchi la barre des 200000 visites mensuelles en novembre 2008. 
- 72 heures pour répondre : il ne s'agit pas tant de fixer une durée qu'un délai, c'est-à-dire donner l'assurance que le questionneur aura une réponse ;

- une réponse précise, pas une orientation;

- les sources sont citées, elles sont vérifiables et fiables ;

- ce sont les bibliothécaires qui répondent, comme experts en validation de sources d'information.

Ces principes n'ont pas varié depuis la création du service, ils semblent être, sinon une recette, du moins les clés du succès du service.

\section{... ET DES INTERDITS !}

Des limites ont été posées, non pas à la nature des questions, mais à la profondeur des réponses pouvant être apportées à certaines questions ou certaines catégories d'usagers.

La première des limites est celle relative aux consultations juridiques et médicales car la loi nous interdit de délivrer des consultations. Si la notion de consultation médicale est assez facile à cerner, celle de consultation juridique l'est moins. La définition admise au sein du guichet est la suivante : est une consultation le fait d'adapter des textes à une situation personnelle. Le GdS doit donc se limiter au renseignement réglementaire. La deuxième limite porte sur les travaux scolaires : le public étudiant ou lycéen peut poser des questions relatives à son travail, mais les réponses fournies se limitent à la bibliographie et la méthodologie de recherche (sources, guides en ligne). Une troisième limite, qui n’a pas été posée au début mais est apparue après l'ouverture est celle opposée aux professionnels de l'infodoc : Le GdS s'adresse au grand public et non aux bibliothécaires. Les principes de fonctionnement du service font l'objet d'une charte d'utilisation (notamment pour signaler les interdictions légales : diffamation, injures racistes, etc.) et sont repris dans la politique du service sur la page d'accueil. 


\section{SCHÉMA UN CIRCUIT DE FONCTIONNEMENT EFFICACE}

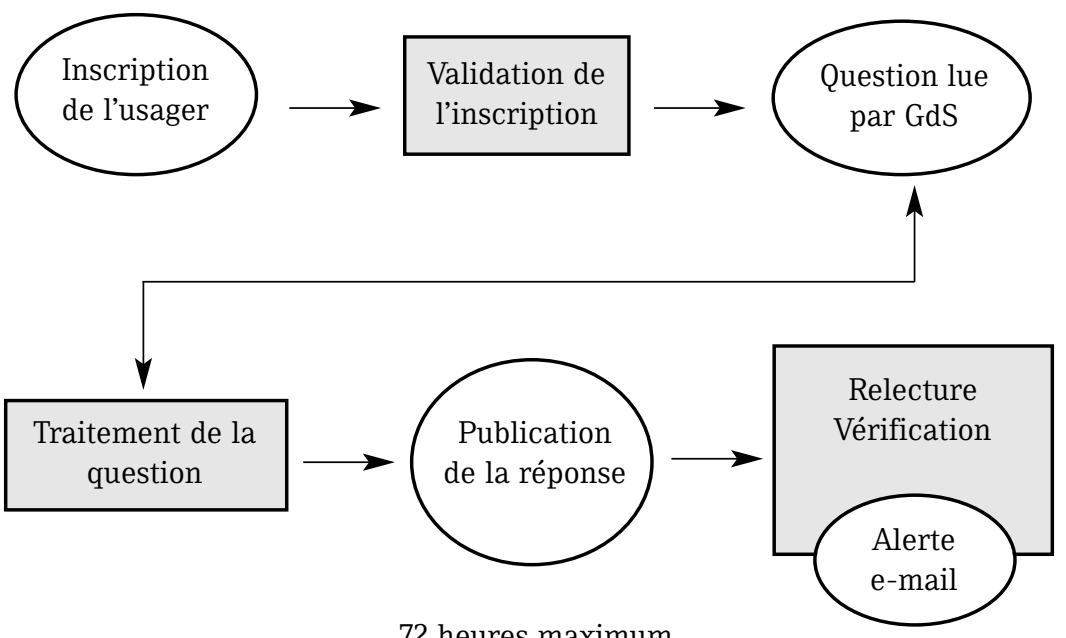

72 heures maximum

Dès qu'un usager s'inscrit et valide son inscription via un e-mail de confirmation, il peut publier sa question, sans modération. Cette très large ouverture n'a valu que très peu de problèmes, seule une dizaine de questions a dû faire l'objet d'un retrait pour des raisons « d'inconvenance » (insultes ou délire pathologique !). La question est lue dès son arrivée par l'équipe du GdS, ou dès l'arrivée de ladite équipe le matin, qui vérifie si elle correspond à la charte. Elle est ensuite adressée (via un code saisi directement dans le corps du message) au département ou service (Le GdS s'auto-adresse le travail de réponse qu'il effectue) qui devra faire une réponse. Le département traite la question comme bon lui semble, dans le cadre défini et publie sa réponse sous 72 heures. Elle est immédiatement publiée, et relue a posteriori par l'équipe du GdS. Le fonctionnement pendant la première année était légèrement différent : les réponses étaient modérées avant d'être publiées. Cette modération a priori a été abandonnée lorsque les principes de réponse ont tous été intégrés par l'ensemble des équipes. La publi- 
cation de la réponse déclenche l'envoi d'une alerte e-mail qui prévient le demandeur qu'il peut consulter sa réponse.

\section{GESTION DU SERVICE}

\section{Les obstacles}

La mise en place du service a nécessité vingt-cinq réunions en trois mois entre responsables de services, chefs de département et comité de pilotage, réunions qui se sont poursuivies la première année entre les répondeurs et l'équipe du Guichet. Les deux principaux obstacles à la mise en œuvre du service ont d'abord été la charge de travail (le service a été ouvert à personnel égal pour les départements), et la légitimité du service, perçu d'abord comme dispensateur d'effort pour les usagers. La première difficulté a trouvé solution d'une part dans la baisse " naturelle » d'activité de la Part-Dieu et dans la recentralisation totale du catalogage auprès du service de la base bibliographique. La seconde objection s'est effacée devant la prise de conscience de la difficulté à trouver des informations fiables à certaines questions, même lorsqu'on est un professionnel de l'information...

L'amplitude horaire n'est pas évidente à définir : le Web ne dort jamais et le service a été conçu pour recevoir des questions 24 heures sur 24. L'équipe $\mathrm{du} G \mathrm{GS}$ est une petite équipe qui assure une amplitude de permanence hebdomadaire de 54 heures. Elle est présente du lundi au samedi, de $9 \mathrm{~h} 00$ à 18 h 00 , par roulement. Le service ne ferme qu'à une seule occasion : la semaine d'inventaire de la Part-Dieu au mois d'août.

Aucune durée limite de recherche n'a été d'emblée déterminée, sinon celle du délai maximal de réponse. Une estimation dans les premiers temps d'ouverture pouvait cependant être faite : une réponse prend en moyenne 40 minutes, encore que la notion de moyenne ne soit pas très pertinente : une réponse peut réellement prendre entre 5 minutes, et 3 jours ! Même si un guide interne de la réponse a été mis en œuvre, il se limite à fixer des principes de contenus (citation des sources, nom du département...) et non de formalisation, ce qui permet de ne pas perdre de temps sur la mise en forme en se concentrant sur le contenu. Il permet aussi de définir un standard commun de réponse, une base qui unifie le service. 
L'équipe du GdS ne dispose que de peu de ressources documentaires propres. À l'exception des recueils de questions-réponses qui sont systématiquement achetés, peu de questions exigent le recours impératif à des documents de référence imprimés dont l'essentiel peut être trouvé en ligne, soit sur le Web, soit dans les bases de données gratuites ou payantes. À quelques exceptions près (toponymie et onomastique, dictionnaires spécialisés), l'essentiel de la référence pour un service généraliste existe aujourd'hui sous forme électronique.

\section{CHOISIR UN OUTIL}

À ce stade de définition du service, la question du choix de l'outil peut-être abordée : le cahier des charges était suffisamment précis pour aiguiller des choix techniques, avec des implications fonctionnelles en terme d'ergonomie, coût, gestion : une interface simplifiée, peu de contraintes liées aux comptes d'usagers, une publication inconditionnelle, des alertes e-mails pour les questionneurs, des niveaux d'usagers différenciés (les questionneurs, les répondants et les modérateurs)... Le choix d'un outil de forum s'est imposé très vite, sur proposition du service informatique, mais nous avons cependant testé d'autres options, avant et pendant l'ouverture du service. Une solution dédiée proposée par un fournisseur de logiciel KM a été étudiée, puis abandonnée, le chat a été essayé en usage public au tout début du Guichet, pendant les premiers mois, mais nous y avons renoncé. QuestionPoint, le logiciel de l'OCLC, a lui aussi fait l'objet d'une expérimentation pendant une année, au sein d'un autre service destiné à des chercheurs sur les pôles d'excellence de la BmL : histoire du livre', documentation régionale et fonds ancien, fonds chinois. L'expérience a été abandonnée en regard du faible nombre de questions reçues ramené au prix de l'outil.

L'outil de forum qui a été choisi, Invision Power Board (IPB) ${ }^{10}$, présente de nombreux avantages : il est presque gratuit (la licence standard coûte maintenant 114 euros à l'année), il est en open source, et il présente un aspect très familier pour des usagers pas nécessairement habitués à des outils 
techniques, avec une ergonomie d'utilisation aisée. Le code en open source a permis à l'équipe informatique de la bibliothèque d'adapter des fonctionnalités nécessaires, l'affichage automatique de la date limite de la réponse dans la barre d'en-tête de la question. On peut inclure dans le corps des réponses des documents, ou des pièces jointes. Enfin, il permet l'extraction de toutes les données sous forme de fichier Excel ce qui simplifie grandement l'évaluation du service. Nombres de données sont récupérables : nombre de questions et de réponses par période choisie, nombre de réponses par service répondant, temps de réponse (non exploité), nombre d'inscrits. Il présente cependant quelques limites : il se prête mal à une éventuelle collaboration inter-établissements, et n'est pas adapté à une utilisation de type « base de connaissances ${ }^{11}$ ". Il correspond par contre tout à fait aux échanges conçus dans le cadre du Guichet.

\section{Des questions et des réponses, quelques exemples en prise avec la réalité}

Le Guichet pour... défendre ses droits en matière d'emploi :

Question : Bonjour!

Je cherche une information et je n'arrive pas à la trouver.

Je travaille dans la métallurgie et dépend donc de cette convention collective en Alsace-Lorraine. Je viens de me pacser et je voudrais savoir si j'ai droit à un ou des jours de congés grâce à ce PACS. Merci d'avance. Lire la réponse : < http://www.guichetdusavoir.org/ipb/index.php?showtopic $=34553>$

Le Guichet pour... trouver de la documentation professionnelle experte :

Question : Bonsoir,

Merci de m'aider à rédiger une information simple et compréhensible par tous salariés sur le risque ATEX pour satisfaire à la nouvelle réglementation.

Lire la réponse : < http://www.guichetdusavoir.org/ipb/index.php?showtopic $=2697>$

11. Il existe bien une base consolidée des archives du Guichet, l'accès thématique, qui est utile et utilisé, mais limité dans sa structuration : < http://www.guichetdusavoir.org/ipb/index.php?c=3 > (consulté le 6 mai 2010). 
Et les « best-sellers » : la mitoyenneté (une cinquantaine de questions environ) ou le contentieux des élections municipales (environ 70 questions), sans oublier la couleur du ciel...

Il ne s'agit que d'un petit aperçu de l'immense richesse des questions, et des réponses postées sur le Guichet. Certes, un service tel que Le GdS ne peut pas être mis en place dans toutes les bibliothèques de par son ampleur. Mais on peut tout à fait imaginer que toute bibliothèque de lecture publique puisse, à son échelle, monter un service de questions-réponses sur au moins un sujet sur lequel elle est sinon experte, du moins spécialisée : son fonds local ou les informations pratiques de proximité. 


\section{3}

\section{SINDBAD, LES PUBLICS ET LES COLLECTIONS DE LA BIBLIOTHĖQUE NATIONALE DE FRANCE

SINDBAD < http://www.bnf.fr/fr/collections_et_services/aides_recherche_ documentaire.html >

\section{LA BNF : BIBLIOTHĖQUE DE RECHERCHE ET TOUS PUBLICS}

Les salles de lecture de la Bibliothèque nationale de France sont fréquentées par des publics différents : tous publics à partir de 16 ans en bibliothèque du Haut-de-jardin, chercheurs en bibliothèque de Recherche. Lors de la mis en place de son service de questions-réponses, la $\mathrm{BnF}$ a choisi de refléter cette double vocation : SINDBAD s'adresse au grand public et aux chercheurs, et met en jeu dans ses réponses à la fois les collections patrimoniales et celles en accès libre. Le service répond à des questions factuelles ou bibliographiques, sur tous les sujets. Afin de ne pas être débordé par le travail de réponse aux questions, il a été décidé de limiter le temps passé à la réponse, en l'annonçant aux usagers.

\section{DES RÉPONSES DE TOUS LES DÉPARTEMENTS}

Entre une équipe dédiée ou la participation de tous les départements de collections, c'est la seconde option qui a été choisie : potentiellement, toutes les personnes qui font du renseignement bibliographique en salle de lecture peuvent répondre à une question SINDBAD.

Cette mobilisation présente deux avantages : sur le plan interne, elle donne à SINDBAD une large assise, en faisant de ce service l'affaire de tous les personnels. Du côté du public, les usagers voient leurs questions traitées par des spécialistes. Les questions sont orientées automatiquement dans les départements en fonction de la thématique cochée par l'usager sur le formulaire de soumissions de la question en ligne ; au sein des départements, un coordinateur répartit les questions selon les compétences de 
chacun : le chargé de collections en philosophie traitera une question sur Aristote, tandis que les spécialistes du monde de l'entreprise se chargeront d'une question sur le marché du bonbon en France.

Un bémol cependant, lié au nombre des répondeurs : la diffusion d'une culture commune de la réponse se fait plus lentement qu'avec une équipe réduite.

\section{UN LIEN FORT ENTRE QUESTIONS ET COLLECTIONS}

SINDBAD reçoit quelques questions qui pourraient être posées à n'importe quelle bibliothèque : "Comment appelle-t-on la figure de style consistant à répéter au début d'une phrase les mots terminant la précédente ? ", "Quelle est la date exacte de la $2^{\mathrm{e}}$ abdication de Napoléon I ${ }^{\mathrm{er}}$ ? »... Cependant, la plupart des questions sont en lien avec les collections de la BnF. Les questions portant explicitement sur les collections représentent environ la moitié des demandes reçues. Ce sont des questions bibliographiques, mais aussi factuelles : "Où puis-je consulter la collection de tarots anciens de la BnF ? », «Avez-vous le numéro de L'Indépendance du 15 juin 1911 ? », "Quelle est la marque du cahier d'écolier utilisé par Beckett pour écrire En attendant Godot ? "... Si on y ajoute les questions bibliographiques qui ne font pas explicitement référence aux collections de la Bibliothèque ( je cherche des articles ou des livres en français sur les piles à bactéries »), mais pour lesquelles les réponses font appel aux collections, on obtient près de deux tiers des questions.

Le nombre de questions reçues en 2009, tous modes de contact confondus (formulaire sur le site < www.bnf.fr >, téléphone, courrier postal) a été de 9 141. La répartition par modes de contact : formulaire site Internet $54 \%$ (4 917), téléphone $45 \%$ (4 152), courrier postal $1 \%$ (72). Sur les 4 ans d'existence de SINDBAD (2006-2009), le nombre moyen de questions reçues par mois est de 835 questions.

Bien que SINDBAD reçoive des questions émanant du " grand public », la plupart sont posées par des chercheurs ou des étudiants. Ce sont des ques- 
tions de spécialistes, dont une partie non négligeable nécessite des vérifications dans les documents: " je voudrais savoir quelles sont les pages consacrées aux éoliennes dans la Revue technique de l'Exposition universelle de 1889 ? ", " le manuscrit syriaque de Rabboula actuellement à la Biblioteca Medicea Laurenziana a-t-il été à un moment de son histoire possédé par la BnF ? »...

Ainsi, bien que le champ d'activité de SINDBAD soit ouvert, on constate que ce service reflète finalement le plus souvent la nature des recherches effectuées sur place par les lecteurs. 


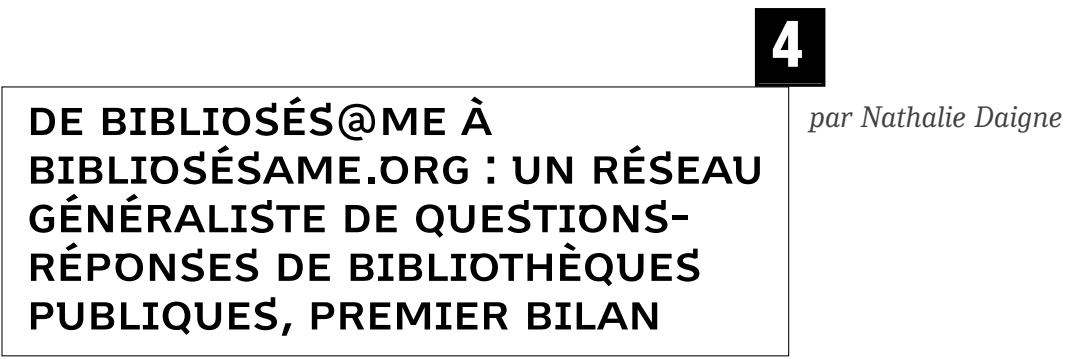

$\Leftrightarrow$ BiblioSésame < http://www.bibliosesame.org >

\section{LA DÉMARCHE PROJET}

En 2003, inspirée par des exemples à l'étranger, la Bpi commence à réfléchir à un réseau de réponses à distance. Elle s'interroge sur ses outils et cherche à introduire le chat dans son service de réponses à distance (RADis). La création du Guichet du Savoir en 2004 accélère cette réflexion : plusieurs bibliothèques municipales s'interrogent également. Autour de la Bpi se constitue alors un groupe de 6 bibliothèques intéressées.

Après beaucoup d'hésitations, c'est le nom «BiblioSés@me »qui est retenu. La décision est prise de s'appuyer sur le prestataire OCLC, et sa plateforme QuestionPoint, et de construire la stratégie de réponses à distance autour du courriel, le chat n'étant testé qu'à la Bpi (surtout pour des raisons financières). Plusieurs scénarios sont envisagés pour la répartition des questions. Finalement, on décide que la porte d'entrée se fera par les sites de chaque bibliothèque, qui renverront au réseau les questions qu'elles ne peuvent traiter. Ces transferts doivent être facilités par l'existence d'une liste des points forts de chaque bibliothèque participante et l'utilisation d'une plateforme commune.

Enfin, la démarche qualité : une charte, qui énonce les grands principes, l'engagement de réponse en 3 jours ouvrés, et ce à quoi le réseau ne répondra pas. Un partage d'expériences est organisé, pour comparer les pratiques et mettre en place une certaine homogénéisation des réponses, dans leur forme et dans leur contenu. Chaque bibliothèque est responsable du contrôle de la qualité de ses réponses et choisit son organisation. Ainsi, à la Bpi, 
c'est le service Accueil et plus particulièrement l'équipe Bibliothèque à Distance qui répond. Un réseau d'experts volontaires est constitué au sein de la bibliothèque, mais il n'est que très peu sollicité, ceux-ci ayant généralement indiqué des spécialités trop précises. Ailleurs, on retrouve le plus souvent une petite équipe gérant les questions, mais avec l'appui de correspondants dans toute leur bibliothèque.

Le réseau commence à fonctionner en 2006 avec 7 bibliothèques : Bpi, Marseille, Troyes, Montpellier, Lille, Valenciennes, Limoges. Arrivent ensuite Reims et Brest en 2007 ; la Bibliothèque Sainte-Geneviève, Amiens, la BDP de Saône et Loire, Toulouse, Strasbourg, la BEI de Cergy en 2008 ; Metz en 2009 ; l'Institut du Monde Arabe et Martigues en 2010. L'arrivée de Caen est également prévue en mai 2010.

\section{FONCTIONNEMENT DU RÉSEAU DE 2006 À 2009}

Comme souvent, le fonctionnement du réseau n'a pas été tout à fait celui qui avait été imaginé au départ. Si le nombre de questions a augmenté régulièrement, cette hausse est restée limitée, ce qui a pu décevoir les bibliothèques. Les questions ont été presque toutes traitées directement par les bibliothèques qui les ont reçues : la possibilité de se transmettre les questions entre partenaires BiblioSés@me est très peu utilisée ; en revanche des questions sont régulièrement réorientées vers l'enssib, à la $\mathrm{BnF}$ et à d'autres partenaires Si@de.

L'existence du réseau s'est donc moins manifestée par la circulation des questions que par la mise en commun d'expériences et d'outils, et par le rôle d'animation de la Bpi.

Être dans le réseau apporte aux bibliothèques une visibilité auprès du public et des professionnels qu'elles n'auraient pas obtenue aussi facilement, sans BiblioSés@me.

La participation à un réseau, et ainsi l'association à sa notoriété et à son image de qualité est souvent un argument efficace pour convaincre les décideurs de se lancer dans un tel projet.

L'appartenance au réseau est assez confortable pour les bibliothèques : c'est la Bpi qui prend en charge les négociations tarifaires, la formation et la plupart des présentations à l'extérieur, c'est elle également qui accompagne 
la réflexion des bibliothèques intéressées. Enfin, elle élabore les statistiques et coordonne l'alimentation de la base de connaissances.

Pour discuter du fonctionnement quotidien et des évolutions à envisager, pour se former et partager les expériences, deux réunions annuelles sont organisées, l'une à Paris et la seconde dans un établissement du réseau. Chaque année, les participants peuvent ainsi visiter un nouvel établissement, découvrir les points forts de son service de réponses à distance et le mode de fonctionnement adopté par son équipe BiblioSés@me.

Un wiki a été créé en 2007 pour rationaliser et faciliter la répartition et le traitement des questions. On y trouve les comptes rendus de réunion, les actualités, les statistiques du réseau ; pour chaque bibliothèque sont indiqués les contacts, les points forts, les abonnements à des bases de données ; enfin une sélection de sites et ressources utiles par thèmes. L'ensemble du contenu est protégé et interrogeable en texte intégral. Nous cherchons maintenant à améliorer l'interface pour la rendre plus conviviale.

\section{9 - 2010 : LA DEUXIÈME VIE DE BIBLIOSÉSAME}

2009 est donc l'occasion d'un premier bilan d'étape. Plusieurs points posent problème : le prix croissant de l'abonnement à QuestionPoint, rapporté à une croissance moindre du nombre de questions, et les difficultés à communiquer efficacement et à valoriser la base de connaissances.

Pour le reste, les bibliothèques restent convaincues par le principe du fonctionnement en réseau et par l'intérêt de ce service qui valorise les ressources des bibliothèques, le savoir-faire des bibliothécaires, et qui semble répondre à un vrai besoin, en touchant un public plus large que celui de leurs lecteurs.

Le réseau décide donc de se doter d'un site fédérateur, d'une voie d'accès simple et facile à mémoriser donnant plus de visibilité et permettant également de toucher plus facilement des internautes qui ne fréquentent pas les bibliothèques.

Un logo est d'abord créé pour donner une identité visuelle commune. C'est l'occasion pour BiblioSésame d'abandonner l'arobase qui compliquait le référencement. Le site < http://www.bibliosesame.org > est ensuite mis en ligne. Il a été voulu le plus clair possible, mettant à l'honneur les biblio- 
thèques et la base de connaissances, en introduisant la recherche en texte intégral ${ }^{1}$. Notre volonté est de rendre cette base accessible aux moteurs de recherche, et d'augmenter ainsi nos chances d'être découverts par hasard. Une campagne de communication est lancée dans la foulée, d'abord en région de novembre à décembre 2009, puis à l'échelle nationale à partir de janvier 2010.

L'organisation initiale est affinée avec la mise en place de ce nouveau site : d'un côté le circuit des questions arrivées sur < bibliosesame.org >, qui sont centralisées et réparties équitablement par la Bpi, en tenant compte si besoin des points forts de chacun, et d'un autre côté les questions qui continuent à arriver directement dans chaque bibliothèque, que celles-ci peuvent toujours renvoyer au réseau si besoin.

Après quelques mois de ce nouveau fonctionnement, plusieurs défis se posent :

- le premier est de renouveler nos modes de communication : pour notre campagne, la presse nationale et régionale a été sollicitée, des marquepages et affiches ont été imprimés, mais c'est véritablement le reportage diffusé en mars à la télévision dans l'émission Télématin qui a permis à notre site d'être découvert plus largement ${ }^{2}$. Comment donc continuer à nous faire connaître ? Comment travailler notre identité numérique, notre présence sur le Web?

- le deuxième défi est d'améliorer nos outils pour gérer l'augmentation $\mathrm{du}$ volume de questions. Il faut en particulier développer ceux qui aident la répartition, en précisant davantage le type de thématiques traitables par les bibliothèques. Le wiki doit également être rénové.

- le troisième pourrait interroger notre fonctionnement interne, en particulier l'animation du réseau d'experts, et les heures de permanence du chat, pour l'instant ouvert deux heures le matin, lorsque la Bpi est fermée : ne pourrait-on pas par exemple envisager de l'ouvrir aux heures d'ouverture de la bibliothèque, pour répondre à tous ceux qui n'ont plus l'habitude de se déplacer jusqu'aux bureaux d'information ou qui ont peur de laisser leurs affaires sans surveillance? 
Nous cherchons également un moyen pour faciliter la communication quotidienne entre tous les répondants. D'autre part, après 5 ans de fonctionnement et des changements dans les équipes, il est temps de formaliser davantage les procédures, le contrôle qualité, l'évaluation statistique, afin de faciliter au maximum la bonne marche de notre réseau. 
5

VOUS AVEZ DES QUESTIONS ?

par Annie Prunet

LES BIBLIOTHÉCAIRES DU RÉSEAU

BIBLIOSÉSAME VOUS RÉPONDENT !

L'EXEMPLE DE LA BMVR DE

MARSEILLE

$\approx$ BiblioSésame < http://www.bibliosesame.org >

UNE PRÉSENTATION SOUS FORME DE QUESTIONS-RÉPONSES

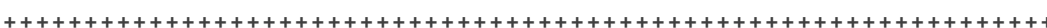

Pourquoi avez-vous intégré le réseau de renseignement à distance

BiblioSésame?

La Direction des bibliothèques de Marseille a toujours reconnu la nécessité d'un service de renseignement à distance. L'ouverture de la bibliothèque de l'Alcazar en 2004 et la mise en place de nouveaux services ont permis de concrétiser ce désir. C'est d'ailleurs un phénomène courant : la création d'un portail, l'ouverture d'un équipement ou l'adoption d'un nouveau fonctionnement sont souvent à l'origine d'un service de questions-réponses. La motivation des bibliothécaires s'appuie sur deux volontés: voir sa bibliothèque exister sur Internet et prolonger la mission de renseignement, rendue de manière quotidienne à la banque d'accueil. Notre rôle peut être délocalisé et démultiplié : nous pouvons exister « hors les murs ».
Que signifie la notion de « collaboratif »? Quel avantage y-a-til à travailler en réseau?

Un réseau collaboratif rassemble plusieurs bibliothèques qui souhaitent travailler ensemble sur l'élaboration d'un projet car il est stimulant d'échanger avec les collègues sur les difficultés, mais aussi sur les petites joies de nos questions / réponses ! L'expérience du « savoir-répondre » de chacun, qui se construit au fil de l'eau, devient profitable à tous ; bénéficier des avantages de la force d'un groupe, car il est rassurant de se sentir accompagné lors de l'ouverture de ce type de service, qui suscite toujours de nombreuses interrogations et aussi parce qu'il existe une saine curiosité : voir comment les autres travaillent et, parfois, s'inspirer de leurs pratiques! Partager ses collections, ses ressources en ligne, ses abonnements à des périodiques régionaux et utiliser le logiciel QuestionPoint d'OCLC. 


\section{Comment s'est constitué le réseau ?}

Les bibliothèques, qui ont tout de suite répondu à l'appel de la Bpi, « attendaient » BiblioSésame : n'ayant pas assez d'effectif pour mener un tel projet seules, elles ont vu en BiblioSésame la solution à leur hésitation, la réponse à leur question... Le réseau s'est constitué de manière progressive à partir de 2006.

\section{Comment s'est mis en place ce} service? Comment vous êtes-vous préparé à cette nouvelle activité ?

À la BMVR de Marseille, après avoir été formés par la Bpi et OCLC, nous avons testé nos compétences : notre service informatique nous a créé une plateforme test et nous nous sommes entraînés à répondre. Par ailleurs, notre expérience enrichie grâce à nos activités au sein de notre service de référence en présentiel, a été très bénéfique : il existe, au final, assez peu de différence entre les questions que nous pose le public en salle et celles que nous recevons via BiblioSésame. Cependant, quand on fait du renseignement à distance, on est lu par le public et par nos pairs : il est donc important de trouver un ton (ni trop familier, ni trop distant), un style (ni trop universitaire, ni trop littéraire), un niveau de réponse et de moduler tout ça en fonction du profil du questionneur, renseigné dans les différents champs du formulaire... La plupart des bibliothèques ont fait une communication régionale mais nous devons surtout rendre ce service visible sur le portail. Macaron sur la page d'accueil, liens de page en page, etc. Tous les moyens seront bons pour faire connaître cette activité. Si l'adhésion à BiblioSésame provoque toujours le jour $\mathrm{J}$ un petit stress, cet évènement demeure dans le souvenir de chaque bibliothécaire un moment assez exaltant.

\section{Quel logiciel utilisez-vous ?}

QuestionPoint. Cette application est reconnue malgré son coût et le manque de convivialité de sa base de connaissances. Elle permet de travailler en réseau : de s'échanger des questions notamment, au sein du même réseau.

\section{Quels moyens humains ont été} affectés ? Quel fonctionnement avezvous adopté?

Les bibliothèques du réseau ont toutes adopté des fonctionnements différents. À Marseille : une équipe de 5 personnes, au sein d'un service de référence, est responsable de l'administration et de la gestion, aidée par des correspondants thématiques ; à Brest, un administrateur assigne les questions à des personnes volontaires ; à Cergy Pontoise, 4 ETP administrent et répartissent aux référents thématiques ; à Lille, un bibliothécaire et un agent de catégorie $C$ répondent aux questions ; à Limoges, une équipe transversale de 7 référents travaille avec 20 correspondants volontaires; à Metz et à Montpellier, 3 personnes répondent ; à Reims, des volontaires sont coordonnés par un responsable. Participer à BiblioSésame s'est rajouté aux tâches des 
bibliothécaires, aucune embauche n'a été faite, les bibliothèques ont réajusté leur fonctionnement et réparti leurs effectifs, souvent en fonction du volontariat du personnel.

\section{Quelle est la nature de vos questions et votre délai de réponse?}

Les bibliothèques reçoivent des questions pratiques, des questions de recherche documentaire (de plus en plus pointues !) et des questions relatives à nos spécialités ; chaque bibliothèque est référente sur des thématiques précises, souvent en rapport avec ses particularités régionales ou avec les points forts de sa collection. Le flux des questions est irrégulier. Il est indéniable qu'un article dans la presse ou une émission à la télévision peut faire monter le nombre des questions de manière fulgurante ${ }^{1}$ ! Les bibliothèques devront peut-être revoir leur organisation au cas où le flux des questions augmente? Le délai de réponse de 3 jours est souvent trop court pour les questions difficiles. Le principe de « tenir les délais » est mineur aux yeux de certains collègues pour qui privilégier la qualité est primordial. Un résultat de recherche est toujours envoyé au bout de trois jours, auquel on peut apporter un complément dans une seconde réponse.

\section{Quel bilan faites-vous de votre partici-} pation à BiblioSésame ?

Les bibliothécaires ont généralement une perception enthousiaste de leur service : un " outil en devenir », " un bilan très positif tant au niveau de la relation avec l'usager [...] qu'au niveau de l'implication professionnelle ». ${ }^{2}$ Il redynamise la fonction « renseignement » et crée une émulation au sein de la bibliothèque ; il nous est parfois arrivé, à Marseille, de solliciter deux ou trois collègues autour d'une même question: se crée ainsi un véritable «bouillon» dans la bibliothèque! Le réseau ne doit cependant plus être partagé entre le désir de recevoir plus de questions et l'appréhension de ne pouvoir assurer la qualité des réponses : la hausse récente atteste de la nécessité de l'existence des services de Q/R. Les bibliothèques doivent s'investir pour satisfaire ce nouveau besoin.

1. Cf. l'article de N. Daigne, «De BiblioSés@me à BiblioSésame.org : un réseau généraliste de questionsréponses de bibliothèques publiques, premier bilan », p. 169.

2. Merci aux collègues du réseau de m'avoir permis d’offrir, grâce à leurs réponses à mes questions, cette illustration régionale de BiblioSésame ! 


\section{6}

UN SERVICE DE QUESTIONSRÉPONSES EN LIGNE DE BIBLIOTHĖQUE SPÉCIALISÉE : BIUMINFO, LE SERVICE DE LA BIBLIOTHĖQUE INTERUNIVERSITAIRE DE MÉDECINE ET D'ODONTOLOGIE (PARIS)

BIUMINFO < http://www.bium.univ-paris5.fr/biuminfo/ >

\section{BIUMINFO EN BREF}

BIUMINFO est un service qui répond toute l'année et gratuitement aux questions touchant tous les domaines de la santé et de son histoire. Il existe depuis 2003 et reçoit entre 50 et 60 questions par mois.

Depuis 2009, BIUMINFO participe aux réseaux Rue des Facs et Si@de. Le trafic en provenance de ces réseaux est actuellement faible. En parallèle, avec l'accès par QuestionPoint installé depuis mars 2010, un accès direct au SRV de la BIUM, bien visible au centre de la page d'accueil du site, est conservé. Un clic donne accès à la charte du service et au formulaire très simple où rédiger sa question. L'anonymat est accepté ; ne sont exigées que l'adresse électronique de l'usager, la mention de son statut (praticiens, étudiants en tel cycle, etc.) et sa situation géographique.

Par souci de conserver une gestion légère et crainte de dévoiler des axes de recherche confidentiels, les questions et leurs réponses ne sont pas rendues publiques.

Les questions sont en majorité bibliographiques (environ $40 \%$ ). Puis viennent les demandes sur la localisation, le prêt, la reproduction d'un document, les questions pratiques (modalités d'inscription, accès distant aux revues, wifi...), les questions factuelles, demandant directement une réponse et non des références, qui se multiplient depuis 2006. Quelques questions de patients nous parviennent occasionnellement. Les questions touchant 
l'histoire de la médecine et les droits sur notre fonds d'images médicales anciennes forment une catégorie à part. Aux plus complexes d'entre elles répondent les personnels de notre département d'histoire de la médecine. Mis à part de rares canulars, aucune question n'est écartée a priori. De même, aucun public n'est écarté $a$ priori. Si les étudiants de $3^{\mathrm{e}}$ cycle médical ou dentaire sont majoritaires, les praticiens en exercice, hospitaliers surtout, sont nombreux à utiliser le service ainsi que les étudiants des filières paramédicales. Nos collègues bibliothécaires/documentalistes représentent une forte minorité des usagers de BIUMINFO. D’autres étudiants, professionnels et particuliers l'utilisent à l'occasion

70 à $80 \%$ des usagers, selon les années, contactent BIUMINFO depuis la France. Ils résident pour les 2/3 hors d'Île-de-France. Peu habitent Paris, où se trouve la BIUM...

Avec chaque réponse est envoyé automatiquement un formulaire de 4 lignes proposant d'attribuer d'un clic un indice de satisfaction de 1 à 4 . Environ $45 \%$ des réponses sont évaluées effectivement, que ce soit via ce formulaire ou par un message de remerciement. Le taux d'évaluation et l'indice de satisfaction élevé sont stables depuis plusieurs années.

L'équipe est composée de 8 agents non dédiés qui signent ou co-signent leurs réponses.

La base est constituée d'un module adapté en interne du logiciel propriétaire FileMakerPro@ utilisé pour d'autres applications de l'établissement, donc peu coûteux et de gestion légère.

À la BIUM du Quartier latin, et sur la BIUM virtuelle, donc aussi sur BIUMINFO, se côtoient des publics relevant de différents champs du savoir, avec différents niveaux de connaissance et d'expérience. À eux tous, sur tous leurs sujets, nous avons pour mission de répondre. Comment?

Dans le présent ouvrage et dans la littérature sont développées des recommandations sur les techniques de réponse, qu'il n'est pas utile de répéter. Cependant, certaines rebondissent d'un article à l'autre, d'une charte en ligne à une autre, mais sur le chemin et au fil du temps, leur justification semble s'être perdue. Il en est ainsi de deux " principes » que nous espérons questionner utilement pour l'avenir des SRV : 
- "répondons mais avec des limites, sans envoyer trop de références ni donner de prime à la paresse, apprenons aux usagers à être autonomes »;

- «valorisons nos collections à travers le SRV ».

Ces énoncés résistent-ils à la pratique d'un service de Q/R spécialisé comme BIUMINFO ?

Nous tenterons de donner une réponse possible à l'aide d'exemples tirés de questions et réponses commentés, et suivis chacun d'une conclusion transposable, nous l'espérons, à d'autres cas et situations.

\section{RÉPONDRE, JUSQU'OÙ ?}

Voilà un exemple représentatif du travail quotidien de l'équipe de BIUMINFO (nous ne reprenons pas les formules de salutations).

Question :

« Je suis interne de spécialité en gynécologie obstétrique et je dois préparer une communication sur la neuropathie pudendale, est-ce que vous pouvez m'aider?»

Réponse :

La base de données EMBASE propose 60 réf. parues depuis 2000 en français ou anglais qui vous sont envoyées par e-mail séparé. La combinaison de termes utilisée est la suivante :

pudendal nerve'/exp/mj AND 'neuropathy'/exp AND ([english]/lim OR [french]/lim).

Si vous souhaitez faire une recherche dans PubMed, faute d'abonnement à EMBASE là où vous êtes interne, de bons résultats sont obtenus avec les termes :

pudendal [tiab] AND (neuropath* OR neuralgia),

en conservant les limites de date (2000-2009) et de langues (français et anglais). Elles sont accessibles en mode " advanced search ».

103 réponses dont 23 revues de la littérature sont proposées, qui vous sont adressées par e-mail séparé.

Si vous avez à votre disposition au SCD ou à l'hôpital la base SCOPUS, vous pourrez comparer. Un essai a été fait avec les mêmes termes et limites de dates et langues : 185 références dont beaucoup se recoupent avec celles d'EMBASE. Je vous envoie les 50 références les plus récentes pour que vous les parcouriez rapidement. 


\begin{abstract}
Vous connaissez sans doute le site français le plus connu consacré à cette névralgie :

< http://www.pudendalsite.com/le-nerf-pudendal.php >

Puisque vous faites une communication, sachez que les sites de Slideshare proposent des présentations sur votre sujet repérées sur le site du Groupe européen de périnéologie :

$<$ http://www.pudendal.com >

Partie : The pudendal nerve: "powerpoint presentation or videos". Plusieurs rubriques intéressantes sur ce site y compris des liens vers des abstracts de congrès sur le sujet et une comparaison en tableau des 3 procédures en usage : trans-périneale, trans-glutéale, trans-ischiorectale, ainsi que quelques images.

Schémas et images sont disponibles sur Google Images et des vidéos sur le bloc du nerf sur Google Vidéos.

Puisqu'il y a plusieurs procédures chirurgicales en usage, une vérification a été faite sur les bases d'essais cliniques de la Cochrane Library. Pas de méta-analyses mais quelques essais ; rien en 2009 ceci dit, ni protocoles en cours. Voici les trois les dernières références : [suivent 3 réf.]

Pas de référentiels HAS. Rien de plus sur le Cismef, ni sur la banque de données de santé publique. Je pensais trouver davantage de références d'evidence-based medicine (EBM ou médecine fondée sur les preuves) sur le sujet. Rien sur le site de la revue «EBM » ni sur le site du groupe BMJ en général.

N'hésitez pas à revenir vers nous si besoin est.
\end{abstract}

\title{
Commentaire :
}

La réponse à cette question très classique se construit en deux temps : - Premier temps : la consultation de plusieurs ressources fiables pour obtenir des données bibliographiques récentes et recoupées (bases de données sur abonnement ou gratuites), le recoupement des données des douze derniers mois étant l'objet d'un soin particulier. La bibliographie est accompagnée des termes de recherche utilisés, que l'usager peut reprendre ou modifier en fonction de sa problématique et de l'avancement de ses travaux. Nous envoyons tous les résultats issus de sources gratuites et une sélection pertinente de celles disponibles sur abonnement payant, au cas où l'usager n'en disposerait pas sur place, ou ne les connaîtrait pas ou mal. 
- Deuxième temps : des sources complémentaires sont utilisées :

- pour indiquer les présentations déjà faites qui pourraient faire gagner du temps à l'usager et lui donner des idées ;

- vérifier l'existence d’images et de vidéos sur le sujet ;

- vérifier les éventuelles connexions du sujet avec des innovations cliniques en cours.

Notons que l'usager n'a pas mentionné ces développements dans sa question. C'est son statut d'interne au sein d'une équipe de recherche et le type de travail qu'il effectue (une communication) qui déterminent le choix et le traitement des sources ainsi que les directions que prennent nos recherches.

Plus généralement : il n'y a pas de limite dans nos envois de références, seulement celles qu'impose le respect des licences d'éditeurs. L'usager reçoit tout ce qui est trouvé, toutes les références, les sources et les liens, avec les mots clés, pour commencer à travailler. Il est informé des ressources consultées sans succès. Le temps consacré à lui répondre n'est pas limité a priori. Pourquoi cet investissement ? Parce que ce service est la seule interface " humaine » de la BIUM virtuelle, avec ses usagers. Il est tout aussi stratégique que les accueils sur place, voire davantage, car il n'est pas substituable. L'usager distant ne peut pas aller d'un agent à l'autre s'il n'est pas satisfait. C'est aussi pourquoi nous proposons en fin de réponse un nouveau contact si le premier n'a pas répondu à l'attente.

Un service de référence est d'abord un service.

Pas une leçon de méthode, pas un outil de formation. Si nos réponses ont des vertus pédagogiques ou méthodologiques, réjouissons-nous ; mais l'objectif premier d'un service, c'est de rendre ce service, c'est (idéalement) d'apporter toute la réponse à la question.

Gardons à l'esprit que nos services de Q/R, dans le monde virtuel, sont en concurrence avec un nombre infini d'autres services ou d'autres sources d'information qui ne se préoccupent pas de pédagogie ; ils répondent. Essayons de faire de même avec notre valeur ajoutée. 
Récemment l'équipe a vécu exactement cette situation. Une traductrice japonais-français nous a soumis cet extrait d'un ouvrage d'un chirurgien pédiatrique des années 1950, Aubaniac : «L'intraveineuse sous-claviculaire supérieure comme voie de secours aux voies fémorale, sinusienne et transosseuse, est indiquée chaque fois que l'intraveineuse au pli du coude est impossible ou insuffisante. » Elle ajoutait dans un français approximatif : « sinusienne est quelle sinusienne ? Est-ce qu'on peut dire « supérieure du sinus sagittal »? En cherchant une réponse adéquate, nous avons remarqué qu'elle avait posé la question sur deux forums de traduction dont un sur lequel les réponses étaient évaluées en vue de récompenses financières. La réponse fournie n'était pas sans fondement mais s'appuyait sur une référence de 2006, peu en phase avec la terminologie chirurgicale des années 50. Nous avons emporté la conviction de la traductrice en lui répondant comme suit :

« Il semble préférable de comparer avec des ouvrages de l'époque d'Aubaniac pour saisir le sens de l'expression. Google Livres propose en ligne un manuel de chirurgie infantile d'urgence écrit en 1958 par Marcel Fèvre qui dit clairement : « chez les petits dont la fontanelle n'est pas fermée, l'injection par le sinus longitudinal supérieur est possible ». Je pense que c'est bien à cette voie sinusienne que Fèvre fait allusion. Aubaniac est chirurgien pédiatrique, ce qui est cohérent avec une technique d'injection via la fontanelle sous laquelle se trouve le sinus longitudinal supérieur ».

\section{LE SRV EST-IL UN OUTIL DE VALORISATION DES COLLECTIONS ?}

Question d'un dentiste senior parisien :

« Je cherche des études statistiques thématiques concernant le contenu des périodiques spécialisés en orthodontie (Revue d'orthopédie dento-faciale, American journal of orthodontics...) ».

Nous lui recommandons l'emploi de GoPubMed, parfaitement adapté grâce à sa fonctionnalité statistics, avec quelques conseils d'usage et des exemples chiffrés d'occurrence (année, pays). 


\section{Commentaire :}

À cette question factuelle d'évaluation statistique des items liés au champ disciplinaire de l'orthodontie, dans les revues spécialisées et sur la durée, répond un outil de comptage libre, aussi simple qu'efficace. L'usager est un professionnel chevronné ; nos quelques indications d'emploi lui ont suffi, nous a-t-il expliqué.

Notons que des éléments factuels chiffrés sont donnés d'emblée dans la réponse, pour capter son attention et le convaincre d'utiliser le produit recommandé. C'est une méthode souvent utilisée dans nos réponses. Donner quelques références récentes très pertinentes tirées d'une liste envoyée par ailleurs par e-mail, que l'usager ne peut pas évaluer tout de suite, lui montre que nous avons bien inclus ses critères et qu'il vaut la peine de travailler sur la liste qui l'attend dans sa boîte à lettres électronique.

Autre question d'un interne au sein d'une équipe de recherche hospitalière :

« J'aimerais savoir s'il existe des sites Web proposant la réalisation de tests statistiques en ligne. Je cherche à calculer le risque relatif d'un facteur pronostique et son intervalle de confiance dans une étude de cohorte ». La réponse proposait trois calculateurs en ligne, trouvés sur un site associatif de partage, un site d'éditeur d'information scientifique, un site privé. L'usager a testé avec succès ces outils et retenu deux d'entre eux.

Notons que dans un cas de ce genre, il convient de proposer si faire se peut plusieurs outils puisque l'enjeu élevé rend appréciable l'opportunité de vérifier un calcul. Notons enfin qu'aucun embargo ne frappe a priori les sites privés : l'essentiel est de donner satisfaction.

Plus généralement, ces deux réponses ne mentionnent à aucun moment une ressource propre à la BIUM. Pourtant, les deux réponses citées ont pleinement satisfait les usagers. N'est-il pas temps de ne plus réduire le service de Q/R à un outil de valorisation des collections ? D'innombrables usagers distants ne viendront jamais dans nos établissements et ne portent que peu ou pas d'attention à l'origine des sources que nous leur indiquons. Dès lors, pourquoi l'expertise des personnels et la veille disciplinaire et documentaire conduite dans l'établissement, compteraient-elles pour rien? Pourquoi 
le service ne valoriserait-il jamais ces éléments constitutifs, parmi d'autres, du statut de la BIUM comme bibliothèque " de référence »? Aujourd'hui, des réponses très bien évaluées par les usagers comportent des liens vers des ressources libres en ligne non labellisées BIUM, ou un mélange des ressources BIUM et non-BIUM, ou, notamment, dans le cas des questions d'histoire de la médecine, uniquement des ressources propres à la BIUM. Toutes, à nos yeux, valorisent l'établissement.

\section{QU'ATTEND L'USAGER ?}

Une réponse complète à sa question, bibliographique ou factuelle, celle que nous voudrions pour nous-mêmes à sa place. Elle se construit en prenant en compte divers critères :

- situation de l'usager (niveau des connaissances, expérience, objectif académique ou professionnel, éloignement géographique des centres de ressources) ;

- pluralité, recoupement, complémentarité des sources de toutes origines, gratuites, sur abonnement disponible à l'université, et sur tous supports (images, vidéos notamment) ;

- investigation transparente et poussée menée avec le recul critique qui vient avec l'expérience des sources déjà connues et une veille continue sur les nouvelles.

Face à l'infini des choix documentaires sur Internet, le service de Q/R est là pour trier, mettre en cohérence, adapter au cas particulier, et présenter un travail complet en toute transparence. 
7

QUESTIONS-SANTÉ,

LE SERVICE DE RÉPONSES EN LIGNE

DE LA CITÉ DE LA SANTÉ

par Sandrine

Lebastard

et Tù-Tâm Nguyên

Questions-santé < http://www.universcience.fr/fr/cite-de-la-sante/ >

\section{UN SERVICE SPÉCIALISÉ EN SANTÉ}

$\mathrm{Au}$ sein de la Bibliothèque des sciences et de l'industrie (BSI), la Cité de la santé est un centre de ressources documentaires et un lieu d'accueil et de service co-animé par une équipe de la BSI et des professionnels de l'information sur la santé mis à disposition par les organismes partenaires.

Depuis octobre 2008, la Cité de la santé propose Questions-santé. Le service fournit gratuitement des réponses personnalisées à toute demande d'information dans le domaine de la santé sans inscription préalable.

Comment Questions-santé se présente sur la page d'accueil du site ? La mise en valeur est différente selon que l'on est sur la page d'accueil de la Bibliothèque ou de la Cité de la santé :

- depuis la page d'accueil de la Bibliothèque $:<$ http:// www.universcience.fr/fr/bibliotheque-bsi > un simple lien vers le service ;

- depuis la page d'accueil de la Cité de la santé : $<$ http://www.universcience.fr/fr/cite-de-la-sante > des liens vers le service, vers le formulaire de question, vers toutes les questions posées et la liste des dernières questions-réponses.

\section{QUI PEUT POSER UNE QUESTION ?}

Toute personne qui cherche de l'information sur la santé, qui est préoccupée par une question de santé, qu'elle soit malade, proche de malade, professionnel de santé ou simple citoyen. 


\section{COMMENT POSER UNE QUESTION ?}

Cliquer sur « Posez votre question » et remplir le formulaire.

\section{QUEL TYPE DE QUESTION PEUT-ON POSER ?}

- les questions de santé et de prévention;

- les enjeux de santé publique ;

- les évolutions des connaissances médicales ;

- les pathologies et leurs thérapeutiques ;

- les handicaps physiques et mentaux : de leur prévention à leur prise en charge ;

- les droits aux soins, la prise en charge et les procédures ;

\section{QUELLE RÉPONSE PEUT-ON OBTENIR ?}

Une réponse personnalisée proposant selon la question :

- des informations spécifiques ;

- des renseignements pratiques ;

- des références documentaires ;

- une orientation vers des associations ou des structures d'accueil, d'écoute, d'information ou de soins.

\section{QUESTIONS-SANTÉ NE FOURNIT PAS :}

- de conseils thérapeutiques ou prescriptions médicamenteuses. Il ne s'agit pas d'une consultation médicale.

- de corrigés, devoirs-types ou bibliographies exhaustives.

\section{QUI RÉPOND ?}

L'équipe de la Cité de la santé : les bibliothécaires et documentalistes, avec le concours de conseillers partenaires : médecins, associations ou professionnels de santé. 


\section{QUELLE BASE DE CONNAISSANCES ?}

Pour l'instant, les questions sont présentées dans l'ordre chronologique inverse et classées par mois. Il est possible de faire une requête à l'aide de la fenêtre « recherche » en langage naturel. À terme, les questions seront indexées par les entrées du référentiel de $<\mathrm{http}$ ://www.universcience.fr $>$.

\section{Questions-santé en bref :}

service asynchrone avec formulaire en ligne ;

4 personnes répondent (soit 2 équivalents temps plein);

50 questions sont traitées par mois ;

délais de réponse : 72 heures (ouvert du mardi au samedi) ;

$80 \%$ des questions/réponses sont publiées sur le site ;

le service utilise Content server (le CMS ou système de gestion de contenu qui gère l'ensemble des ressources de < http://www.universcience.fr >).

\section{QUelQues TYPeS DE QUeSTIONS POSÉES}

- Recherche d'information pour une prise de décision personnelle : «Bonjour, Je dois vacciner mon bébé contre l'hépatite B. Elle a déjà reçu une première injection mais hier, j'ai vu un documentaire sur le sujet et je suis inquiète. Mon médecin me dit que lorsqu'on vit en IDF, il est préférable de vacciner. Le documentaire expliquait que le nombre de personnes touchées par des effets secondaires est en augmentation. Quel est réellement le pourcentage de personnes touchées par l'hépatite et quel est celui des personnes qui subissent les effets secondaires du vaccin?»

- Recherche d'information pour mieux dialoguer avec son médecin :

"Pouvez-vous me donner les dernières techniques pour une intervention de cardioversion externe arythmie et fibrillation ventriculaire. Pas encore vu mon cardiologue, J'aimerais avoir quelques éléments afin de pouvoir discuter utilement avec lui. »

- Demande d'explication sur un phénomène physiologique :

« D'où viennent les cheveux blancs?» 
- Informations sur l'état de la recherche sur une pathologie ou sur un traitement :

« Où en est-on concernant le traitement de l'arthrose ? »

- Témoignage polémique :

"Que pensez-vous de 35 ans d'acharnement psychiatrique sur mon épouse à base de toute sorte de "produits chimiques" et particulièrement de "ZYPREXA" qui ont détruit notre vie familiale, et surtout la santé de ma femme...??? Suite à une TS en 2003, j'ai essayé de sensibiliser une trentaine de médias, aucune réponse... ! J'ajoute que tous ces traitements, imposés, malgré ma réprobation, et contre ma volonté, n'ont jamais résolu ses problèmes et n'ont fait qu'aggraver son état et qui se retrouve comme une loque de $38 \mathrm{~kg}$. !!! »

- Recherche d'adresse de spécialistes, de lieux où l'on pratique une technique particulière :

«Une parente va se faire opérer d'un méningiome olfactif et souhaiterait savoir quels sont les neurochirurgiens spécialisés, s'ils existent, qui savent le faire ?»

- Recherche d'information dans le cadre d'un travail scolaire :

«Comment les substances dopantes se propagent-elles et agissent-elles sur l'organisme? »

- Recherche d'un consensus des scientifiques sur une problématique :

" J'ai lu des informations inquiétantes sur le fait de chauffer de la nourriture au four à micro-ondes ; notamment une étude scientifique suisse (cf. Hertel et Blanc) très alarmante. Quelle est la position actuelle des scientifiques : sont-ils divisés sur le sujet ? La consommation d'aliments chauffés au micro-ondes est-elle nuisible à la santé ?»

Un exemple (les formules de politesse ont été supprimées) :

Question

Est-ce qu'il y a du blanc d'œuf dans la composition du vaccin ? Et si oui comment dois-je faire pour me vacciner? 


\section{Réponse}

Vous souhaitez savoir si les vaccins contre la grippe A (H1N1) contiennent de l'œuf et comment se faire vacciner lorsque l'on est allergique à l'œuf.

Selon le réseau InfoVac (un réseau d'experts indépendants des laboratoires pharmaceutiques et mis à disposition pour faciliter l'accès des médecins aux questions liées aux vaccinations), « Le virus A H1N1/09 est produit soit sur des œufs (Pandemrix de GlaxoSmithKline, Focetria de Novartis vaccines, Humenza de Sanofipasteur, etc.) soit sur des cellules en culture (Celtura de Novartis Vaccines, Celvapan de Baxter, etc.). Il est ensuite inactivé (tué) et fragmenté pour récupérer les protéines de la surface du virus - celles contre lesquelles les défenses immunitaires doivent être dirigées ».

< http://www.infovac.ch/index.php?option=com_content\&view=article\& $\mathrm{id}=682 \&$ Itemid $=528$ >

\section{Culture sur des œufs :}

On retrouve effectivement dans la composition des vaccins Pandemrix (laboratoire GlaxoSmithKline Biologicals) et Focetria (Laboratoire Novartis Vaccines and Diagnostics) que la culture de l'antigène a été faite sur des œufs :

- notice Pandemrix : < http://www.emea.europa.eu/humandocs/PDFs/ EPAR/pandemrix/emea-combined-h832fr.pdf >

- notice Focetria : < http://www.emea.europa.eu/humandocs/PDFs/EPAR/ focetria/emea-combined-h710fr.pdf >

- le vaccin Panenza (laboratoires Sanofi Pasteur) qui vient de recevoir son autorisation de mise sur le marché est un « vaccin sans adjuvant, produit sur œufs embryonnés. Il contient 15 microgrammes d'antigène par dose de $0,5 \mathrm{ml}$ de vaccin. Le vaccin sera disponible en présentation unidose à $0,5 \mathrm{ml}$ et multidoses avec présence d'un conservateur (thiomersal)».

< http://www.afssaps.fr/Infos-de-securite/Communiques-de-presse/ Autorisation-de-mise-sur-le-marche-du-vaccin-pandemique-Panenza/ (language)/fre-FR >

\section{Production sur des cellules en culture :}

En ce qui concerne le vaccin Celvapan (des laboratoires Baxter) la culture de l'antigène a été faite sur des cellules Vero (lignée cellulaire continue d'origine mammifère) :

- notice Celvapan : < http://www.emea.europa.eu/humandocs/PDFs/EPAR/ celvapan/emea-combined-h982fr.pdf > 
190 | Mettre en œuvre un service de questions-réponses en ligne

Il paraît de toute façon plus prudent de demander l'avis d'un médecin avant toute décision de vaccination. C'est ce que rappelle le site Infovac : «Les personnes avec des réactions allergiques graves aux protéines de l'œuf ou aux composants des vaccins ne devront pas être vaccinées avant d'en avoir discuté avec leur médecin traitant. » < http://www.infovac.ch/index.php?option=com_content\&view=article\& id $=682 \&$ Itemid $=528>$

Nous espérons que ces informations vous seront utiles et restons à votre disposition pour tout complément.

L'équipe de Questions-santé,

Le service de réponses en ligne de la Cité de la santé.

Service Questions-santé

< http://www.universcience.fr/fr/cite-de-la-sante/ > 


\section{8 \\ LE SERVICE QUESTIONS ? par Pierre Moison RÉPONSES! DE L'ENSSIB}

$\Leftrightarrow$ Service Questions ? Réponses ! < http://www.enssib.fr/questionsreponses/ >

Ouvert en octobre 2007, le service Question ? Réponses ! est un service de questions-réponses en ligne spécialisé dans les sciences de l'information et des bibliothèques. Il répond à une forte demande, puisque la Bpi et Le $\mathrm{GdS}$ recevaient déjà un grand nombre de questions relevant de ce domaine. Ce sont les bibliothécaires de l'école qui répondent, dans un délai de 3 jours ouvrés. Les réponses alimentent une base de connaissances et donnent matière à des produits documentaires.

Le service est ouvert à tous, à l'exclusion des élèves de l'enssib. Les questions proviennent principalement de professionnels de la documentation (40\%) et d'étudiants (35\%). $15 \%$ des questions viennent de l'étranger. Les utilisateurs recherchent soit l'avis d'experts (une information validée), soit l'avis de pairs (des conseils, des retours d'expérience). Le service répond en moyenne à 60 questions par mois. En mars 2010, les répondeurs ont fêté la $1000^{\mathrm{e}}$ question traitée depuis l'ouverture du service.

L'équipe est constituée d'un coordinateur et de 10 bibliothécaires de catégorie $A$ et $B$ du département des services aux bibliothèques, pour lesquels la participation au service questions-réponses n'est pas la mission principale. Néanmoins, la participation au service est considérée comme prioritaire et inscrite dans les fiches de postes des répondeurs. La taille modeste de l'équipe facilite la coordination et le partage des connaissances. Les répondeurs sont répartis en 6 groupes thématiques, selon les compétences et l'intérêt de chacun. Chacun participe à plusieurs groupes, ce qui permet d'avoir sur chaque thématique un nombre suffisant de répondeurs potentiels. Par ailleurs, des réunions hebdomadaires réunissent toute l'équipe 
pour permettre à chacun de se familiariser avec les champs qu'il maîtrise le moins et d'être polyvalent pour répondre à tout type de question le cas échéant.

La modération est assurée en alternance par le coordinateur et deux chefs de service. Le modérateur est prévenu de l'arrivée d'une nouvelle question via sa messagerie et l'assigne au chef du groupe concerné, qui sollicite un répondeur et un relecteur. Une fois la question rédigée et relue, elle est envoyée à l'usager par le modérateur. Ce circuit est relativement long, mais il permet une concertation à différentes étapes et garantit la qualité des réponses, le dialogue et les éclairages multiples autour d'une même question permettant d'identifier des problématiques qui auraient pu être négligées par un répondeur isolé. En cas de besoin, le modérateur peut solliciter l'avis de correspondants au sein de l'école (un par département : informatique, scolarité, personnel, finances, éditions, recherche). Parallèlement, le modérateur alimente un tableau qui lui permet de suivre les questions en cours et de collecter des statistiques (temps de réponse, nombre de questions traitées par mois, typologie des questions). Chaque mois, une dizaine de questions sont transmises par d'autres services de référence (principalement BiblioSésame et SINDBAD) ; nous réorientons également régulièrement certains des utilisateurs vers d'autres services mieux adaptés - le plus souvent via le label Si@de.

\section{DES RÉPONSES POUR DES SPÉCIALISTES}

Dans la mesure où le public est constitué à la fois de professionnels de l'information et d'étudiants, les réponses proposées oscillent entre le souci du factuel propre aux services de référence de lecture publique (fournir des éléments de réponse en épargnant la recherche à l'utilisateur) et la référence de type universitaire (renseignement bibliographique et pédagogie documentaire). Nous veillons à adapter la réponse en fonction des besoins et des compétences propres à chaque utilisateur. Pour ce faire, une attention particulière est portée au contexte de la demande et aux enjeux implicites. Si nécessaire, nous reformulons la question en début de réponse, de façon à clarifier ou à expliciter la manière dont nous l'avons comprise. Cette 
reformulation permet d'indiquer la manière dont nous allons répondre : si la question est vaste, nous précisons quels points seront abordés en priorité ; à l'inverse, nous pouvons proposer d'élargir le champ du questionnement pour resituer la question au sein d'une problématique plus large. Nous veillons à proposer d'emblée l'information la plus pertinente au regard de la demande et à présenter les références de façon normalisée. Nous privilégions les sources primaires. Enfin et surtout, nous nous efforçons de citer les passages qui répondent précisément à la question. Si nos réponses ne visent pas d'emblée à former les usagers à la recherche documentaire (bien que la méthodologie de la recherche soit évoquée), leur valeur pédagogique transparaît dans la manière dont nous nous efforçons de les structurer. Produire de telles réponses demande du temps : les répondeurs passent en moyenne 1 heure et demie par réponse.

La participation au service constitue une expérience professionnelle particulièrement riche pour les répondeurs, qui développent des compétences en matière de référence tout en se formant sur les thématiques abordées. Une attention particulière est accordée à la formalisation des méthodes de réponse. Des réunions qualité hebdomadaires permettent d'évoquer les réponses envoyées ou en cours (problématique retenue, stratégie de recherche, ressources consultées, structuration de la réponse...) et d'harmoniser ainsi nos pratiques. La formation de l'équipe est également assurée par la présentation d'outils documentaires et de sources d'information, ainsi que par une veille sur les problématiques émergentes. Un espace partagé permet de rassembler les règles de réponses et de répertorier les ressources utiles pour chaque thématique. Un bureau est également affecté à l'équipe, dans lequel sont rassemblés des ouvrages de référence ainsi que des dossiers documentaires. Enfin, des réunions de suivi mensuelles permettent de présenter des ressources utiles pour les répondeurs et de faire évoluer le fonctionnement du service.

\section{LA BASE DE CONNAISSANCES}

Les réponses sont archivées et rendues publiques au sein d'une base de connaissances qui propose près de 600 réponses. Cette base peut être explo- 
rée via une recherche sur le texte intégral ou via un index thématique, les réponses étant indexées à l'aide de la classification mise en place pour la bibliothèque numérique de l'école. Des fils RSS sur chaque thématique permettent à l'usager d'être tenu informé dès qu'une nouvelle réponse est publiée. Les réponses sont datées mais ne sont pas mises à jour ni désherbées, ce corpus constituant un témoignage précieux des questionnements de la profession et de ses besoins en matière de formation. Enfin, les répondeurs valorisent l'expertise acquise sur certaines questions en élaborant des Fiches pratiques pour la bibliothèque numérique ${ }^{1}$.

Mais ce qui fait la spécificité de ce service, c'est que ses usagers disposent de compétences proches de celles des répondeurs. Si cette situation permet une certaine connivence, elle implique surtout pour les répondeurs de faire preuve d'un discernement particulier et d'être au fait des évolutions les plus récentes du contexte professionnel. 


\section{MÉMENTO}

par Claire Nguyen

\section{MONTER SON PROJET DE SERVICE}

\section{RÉFLÉCHIR AUX PRÉALABLES}

- Étudier la demande et les besoins. L'analyse des besoins doit se faire selon la nature et la taille de la bibliothèque, le profil de ses usagers. Choisir sa méthodologie : observation directe, entretien, questionnaire d'enquête.

- Étudier les services existants : aller à des journées d'étude, parler aux professionnels ; faire une revue de la littérature professionnelle. Étudier les autres services de questions-réponses existants (benchmarking).

- Avoir une vision et une stratégie pour son service.

- Intégrer le projet aux autres projets de l'établissement : il peut faire partie d'un projet plus global (refonte du site Web) ou exigé par des circonstances particulières (fermeture de l'établissement, etc.) ; identifier les services qu'on valorisera également via le nouveau service (collections spécialisées, fourniture de document, formations, etc.)

\section{METTRE EN PLACE UN GROUPE ET UN PLAN DE PROJET}

- Obtenir une lettre de mission de la direction ou des instances de l'établissement.

- Mettre en place un groupe projet (désigner un chef de projet).

- Rédiger un plan projet et définir un projet-pilote : proposer les outils ; proposer des scénarios ; évaluer la charge de travail, déterminer le personnel impliqué et les responsabilités attribuées, la gestion des risques. Choisir ou non une assistance à maîtrise d'ouvrage.

- Évaluer les coûts : sur quel budget est financé le projet (récurrent, exceptionnel) ? Planifier les coûts sur plusieurs années (frais de développement, amortissement) ; évaluer les coûts de personnel, les coûts de l'espace, des fournitures, de l'achat de documentation; le coût de la solution technique.

- Établir le calendrier et la planification.

- Se référer aux normes. 
- Faire une étude de faisabilité : état des lieux, articulation avec le service en présentiel ; recensement du personnel et leurs compétences, inventaire des ressources documentaires existantes, l'infrastructure.

- Présenter et négocier le projet.

\section{ORIENTER ET ORGANISER SON SERVICE}

- Définir la politique de service : missions, engagements et périmètre du service (public, nature des questions, niveau de réponse, délais). Choisir ou non de publier les questions/réponses ; déterminer l'ouverture du service (réservé, ouvert à tous, gratuit ; payant, etc.)

- Rédiger et valider par tous la charte interne et externe du service en termes clairs et compréhensibles.

- Choisir un modèle d'organisation adapté, en réfléchissant bien au mode de recrutement, au nombre de répondants, aux plannings, au lieu d'où l'on répond, aux périodes pendant lesquelles on répond.

- Ne pas déséquilibrer le fonctionnement global de la bibliothèque (réguler les flux) si les autres services sont sollicités.

- Organiser le service en interne : acquérir les compétences des répondants et du coordinateur (savoir-répondre; chercher et sélectionner l'information, maîtriser l'interface, autres compétences techniques) ; organiser les formations pertinentes ; organiser des réunions régulières de débriefing ; choisir les supports internes (documentation papier, répertoire de signets, wiki...) ; établir un circuit de réponse schématisable.

\section{ChOISIR SES OUTILS}

- Se poser les questions sur ses besoins, ses moyens, ses souhaits.

- Les modes de réponse : asynchrone (courriel, formulaire Web, SMS, blog) / synchrone (plateforme web, messagerie instantanée (avec des fonctionnalités audio-vidéo). Faire de la veille sur les nouveaux outils. Peser les avantages et les inconvénients de chaque outil.

- Établir une grille comparative des différentes solutions existantes.

- Si on choisit un logiciel libre, penser aux développements nécessaires auprès des prestataires. 


\section{MONTER UN RÉSEAU DE QUESTIONS-RÉPONSES COLLABORATIF}

- Développer des partenariats (bibliothécaires, professionnels, experts...) formalisés ou non dans un réseau.

- Penser à l'articulation entre service local et collaboratif.

- Se servir ou non d'une infrastructure déjà existante (ex : RUOA pour Ubib.fr) ou monter un service ex nihilo. Bien définir le périmètre de la collaboration (thématique, géographique, etc.).

- Monter un comité de pilotage qui définira la stratégie et les orientations politiques ; un ou plusieurs groupes de travail (techniques). Choisir un logiciel collaboratif, une politique de service (cf. supra).

- Élaborer l'architecture du réseau (ex : organisation en fonction de " pétales » thématiques).

- Recenser les répondants et évaluer leurs compétences; organiser les formations.

- Organiser la répartition du travail, qui peut être différente selon le mode de réponse (synchrone, asynchrone).

- Mettre en place des outils collaboratifs accessibles à distance (notamment annuaire des bibliothécaires, répertoires de ressources, signets, blog, mise à disposition des statistiques).

\section{PROMOUVOIR}

- Identifier le public ciblé par la promotion (étudiants, enseignants chercheurs, grand public), les modes de communication (e-mails, affiches.), les lieux de la promotion (formation, bureau de renseignement). Si on lance une campagne de communication, choisir son moment et les médias (institutionnels, locaux, nationaux; presse écrite ou télévision...).

- Soigner son image de marque (choix du nom du service, logo à protéger, goodies) ; penser à l'aspect visuel du service.

- Estimer les coûts de la campagne de communication.

- Marquer sa présence en ligne (widget...) et disséminer le service (ENT, blogs, réseaux sociaux, portails).

- Se faire voir : intégrer le service dans l'environnement de l'usager.

- Penser aux formalités d'accès du service. 
- Réfléchir aux renseignements demandés à l'usager (éléments nécessaires pour le contact, autres éléments pour cibler son profil ; anonymat ou non).

\section{INTÉGRER LE SERVICE AUX AUTRES SERVICES DE LA BIBLIOTHĖQUE}

- Considérer le service comme nécessaire ainsi que la recherche ; la validation de l'information, la rédaction de la réponse comme participant à la formation des personnels.

- Produire des contenus documentaires à partir des questions-réponses.

- Intégrer les contenus dans l'offre documentaire (catalogue, signets...).

\section{CONSTRUIRE SA RÉPONSE}

- La méthodologie peut être écrite dans la charte interne du service et/ou dans un guide de réponse. Ce guide prendra en compte les différents modes de réponse.

- Il faut se mettre d'accord sur : la forme visuelle de la réponse (structure visible et formalisation...), la stratégie de la réponse (fournit-on la réponse ? Détaille-t-on la méthodologie de recherche ?), le style de la réponse, les invariants (formules d'introduction, de conclusion, signature standardisée...).

- Ne jamais laisser une question sans réponse même s'il s'agit d'une réorientation.

- Lecture attentive, compréhension, clarté, précision, concision sont les maîtres-mots.

- Adopter une méthodologie de recherche. Penser à chercher des documents de tout type (multimédia, documents gratuits ou sur abonnement, signets de la bibliothèque, etc.).

- Si on propose une base de connaissances aux usagers : travailler sur les questions-réponses (corrections orthographiques, inclusion ou non des formules de politesse...) ; choisir de sélectionner les questionsréponses ou de tout publier ; indexer les questions-réponses ; choisir le mode d'interrogation (feuilletage, arborescence, interrogation plein texte). 
- Respecter le cadre juridique : l'accord de la publication de la réponse, le droit d'auteur, le droit de citation, garantir l'anonymisation des données du lecteur, ne pas faire d'exercice illégal de la médecine et du droit.

\section{ÉVALUER}

- Choisir des indicateurs quantitatifs, selon chaque mode de réponse. Se référer aux normes ISO 2789 et ISO 116620 - pas toujours complètes pour bien évaluer les services de $Q / R$.

- Mesurer la satisfaction de l'usager (questionnaires...) ; on peut aussi se référer aux recommandations existantes.

- Évaluer la qualité des réponses (cf. guides de la RUSA) sur une période déterminée ; coder les transactions après avoir catégorisé les questions, les usagers et traitements de la réponse.

- Évaluer la notoriété du service (questionnaire administré ou entretiens collectifs).

- Évaluer la satisfaction des bibliothécaires.

- Choisir ses outils d'évaluations (tableurs, logiciels de sondage, etc.).

- Tirer des conclusions de l'évaluation pour améliorer le service.

\section{EN CONCLUSION}

Comme en témoignent toutes les contributions de cet ouvrage, les services de questions-réponses en ligne ont atteint une certaine maturité, tant dans les bibliothèques académiques que publiques. L'offre de services est devenue significative et beaucoup de modèles sont et ont été expérimentés. La volonté et la constance, représentées par l'impulsion politique de sa direction, l'investissement de l'établissement et l'implication des personnels, sont les éléments moteurs de ces projets. C'est pourquoi les freins, les difficultés ou les réticences ne constituent pas de réels obstacles pour mettre en place un service de questions-réponses, à petite ou grande échelle. 
200 


\section{SIGLES ET ACRONYMES}

ACRL : Association of College and Research Libraries

ALA : American Library Association

AENES : Administration de l'éducation nationale et de l'enseignement supérieur

BAL : Boîte aux lettres électronique (dans cet ouvrage)

BC : Base de connaissances

BDP : Bibliothèque départementale de prêt

BmL : Bibliothèque municipale de Lyon

BnF : Bibliothèque nationale de France

BR : Bureau physique de renseignement

BU : Bibliothèque universitaire

CADIST : Centre d'acquisition et de diffusion de l'information scientifique et technique

CHSCT : Comité d'hygiène, de sécurité et des conditions de travail

CMS : Content management system / système de gestion de contenu permettant de concevoir et développer des sites Internet.

CNIL : Commission nationale de l'informatique et des libertés

CNRS : Centre national de la recherche scientifique

CP : Comité de pilotage

CRI : Centre de ressources

informatiques

DADVSI : Loi relative au droit d'auteur et aux droits voisins dans la société de l'information

ENT : Environnement numérique de travail

ESGBU : Enquête statistique générale auprès des bibliothèques universitaires de l'enseignement supérieur

FAQ : Foire aux questions

GdS : Guichet du Savoir

GT : Groupe de travail

IFLA : International Federation of Library Associations and Institutions 
IM/MI : Instant messaging /messagerie instantanée

ISO : Organisation internationale de normalisation

ITRF : Ingénieur et personnel technique de recherche et de formation

KM : Knowledge management / gestion des connaissances)

MIT : Massachusetts Institute of Technology

OCLC : Online Computer Library Center

Service de Q/R (en ligne) : Service de questions-réponses (en ligne)

OPAC : Online Public Access Catalog

PEB : Prêt entre bibliothèques

PRCE : Professeur certifié affecté dans l'enseignement supérieur, dans une université, un IUT ou un IUFM

PRES : Pôle de recherche et d'enseignement supérieur QP : QuestionPoint (logiciel d'OCLC) REL : Service de renseignement en ligne
RENATER : Réseau national de télécommunications pour la technologie, l'enseignement et la recherche

RISS : Reference and Information Services Section

RU0A : Réseau des universités de l'ouest Atlantique

RUSA : Reference and Users Services Association

SCD : Service commun de la documentation

SICD : Service interétablissements de coopération documentaire

SMS (ou texto) : Short message service

SPAM : Message électronique non sollicité

SRV : Service de référence virtuel

UFR : Unité de formation et de recherche

UNR : Université numérique régionale 


\section{GLOSSAIRE}

\section{Agrégateur (de contenu)}

Application agrégeant et synthétisant des informations venant de plusieurs sites internet.

\section{API-XML}

(Application Program Interface - XML) Interface de programmation d'application permettant aux développeurs la création, la manipulation et le traitement de fichiers XML pour construire des requêtes qui seront lancées au niveau d'une interface Web (site Web par exemple) (d'après J.-C. Houpier).

\section{Benchmarking}

Démarche d'analyse et d'observation des meilleures pratiques employées par la concurrence et les autres secteurs sur le sujet étudié (d'après J.-P. Accart).

\section{Blog ou blogue}

Site Web constitué d'une succession de billets constitués au fil du temps, classés du plus récent au plus ancien.

\section{Chat}

Mode de conversation instantanée en ligne sous forme de texte.

\section{Firewall (pare-feu)}

Logiciel permettant de sécuriser le réseau; contrôle les communications entrantes et sortantes.

\section{Flux RSS}

Flux de contenus gratuits en provenance de sites Internet.

\section{Goodies}

Objets de la vie courante (pot à crayon, stylo) frappés du logo d'une marque (d'après N. Alarcon), produits dérivés.

\section{HTML (HyperText Mark-Up Language)} Langage de création de pages Web utilisant une structure faite de balises pour mettre en forme le texte.

\section{Plug-in (plugiciel ou greffon)}

Logiciel complétant un autre logiciel, pour lui apporter de nouvelles fonctionnalités.

\section{Portail}

Espace de communication sur Internet à destination d'une communauté définie.

\section{Proxy}

Serveur intermédiaire entre un client HTTP et un serveur, permet la sécurisation de transmission de données dans un réseau local.

\section{Résolveur de lien}

Outil proposant des services contextuels au niveau d'une référence bibliographique tels que l'accès direct au texte intégral, l'accès à la référence bibliographique du document ou à sa version imprimée, l'accès à des ressources et services complémentaires (d'après J.-C. Houpier). 


\section{Voix sur réseau IP (voIP)}

Technique permettant de téléphoner via Internet.

\section{Widget}

Contraction de windows (fenêtre) et de gadget, c'est une fenêtre facilement importable dans un site Web (d'après N. Alarcon).

\section{Workflow (gestion de flux)}

Flux d'informations au sein d'une organisation.

\section{XML (eXtensible Markup Language)}

Langage à balises étendu ou langage à balises extensible. Langage de description de documents (sorte d'HTML amélioré), utilisant des balises personnalisées et l'échange de données. 


\section{BIBLIOGRAPHIE}

Accart Jean-Philippe. Les services de référence : du présentiel au virtuel. Paris, Éditions du Cercle de la librairie, 2008 (Collection Bibliothèques), 283 p.

Alarcon Nicolas. « 2009, année des services de renseignements en ligne ? ». BIBLIOthèques - revue de l'ABF, n 46 , oct. 2009, p. 76-77.

American Library Association. Cooperative Reference Service Committee Reference and User Services Association. Guidelines for Cooperative Reference Services. Nov. 2005. Guidelines for Behavioral Performance of Reference \& Information Services Professionals. Updated June 2004. Guidelines for Implementing and Maintaining Virtual Reference Services. Updated 2009. In American Library Association. [En ligne]

< http://www.ala.org/ala/mgrps/divs/ru sa/resources/guidelines/index.cfm > (consulté le 12 avril 2010).

Arnold Julie, Kaske Neal. "Evaluating the Quality of a Chat Service". Portal: Libraries and the Academy, 2005, vol. 5, $\mathrm{n}^{\circ} 2$, p. 177-193.

Association française de normalisation. Indicateur de performance des bibliothèques, information et documentation : norme ISO 11620. Paris, Afnor, 2008.
Association française de normalisation. "Management de la qualité, enquête de satisfaction des clients ", in Norme FDX 50-172. Paris, Afnor, 1999.

Association française de normalisation. Statistiques internationales de bibliothèques : information et documentation : ISO 2789:2006 =Information and documentation: International library statistics. [Saint-Denis-La-Plaine] : AFNOR, 2006.

Benoist David. « Référence virtuelle. Quel rôle face aux moteurs de recherche? ". Bulletin des bibliothèques de France, 2007, t. 52, n 6, p. 25-27. [En ligne] < http://bbf.enssib.fr >

Breitbach Will. "Using Jing to Turn your IM or Chat Reference into Multimedia Educational Experience”. $9^{e}$ Brick \& Click Libraries Symposium. Maryville, 2009.

Calenge Bertrand. " Les Bm à la recherche de leurs usagers ». Bulletin des bibliothèques de France, 2003, t. 48, $\mathrm{n}^{\circ}$ 1, p. 88-90. [En ligne] < http://bbf.enssib.fr >

Calenge Bertrand, Di Pietro Christelle. « Le Guichet du Savoir ${ }^{\circledR}$ ». Bulletin des bibliothèques de France, 2005, t. 50, $\mathrm{n}^{\circ} 4$, p. 38-42. [En ligne] < http://bbf.enssib.fr > 
Connaway Lynn Silipigni, Radford Marie L., Dickey Timothy J. "On the Trail of the Elusive Non-user: What Research in the Virtual Reference Environments Reveals". Bulletin of the American Society for Information Science \& Technology, 2008, vol. 34, $\mathrm{n}^{\circ} 2$, p. 25-28. [En ligne]

< http://www3.interscience.wiley.com/ journal/121410876/abstract?CRETRY=1\& SRETRY=0 > (consulté le 12 avril 2010).

\section{Evans Christophe, Peyrelong Marie-} France. "Quand les professionnels de l'information redeviennent utilisateurs ". Bulletin des bibliothèques de France, 2010, t. 55, n 4, p. 67-75. [En ligne] < http://bbf.enssib.fr >

Fournis Yves. Les études de marché : techniques d'enquêtes, sondages, interprétation des résultats. Paris, Dunod, 1995.

Grodzins Lipow Anne. The virtual reference librarian's handbook. New York, Neal-Schuman, 2003.

\section{International Federation of libraries associations and institutions. Section du travail de référence. " Lignes direc- trices de l'IFLA en matière de référence numérique ». [En ligne]. In IFLANET [Site]. Section du travail de référence, 2002-2003. \\ < http://www.ifla.org/VII/s36/pubs/ drg03-f.htm > (consulté le 12 avril 2010).}

Izenstark Amanda K. "Choosing the Right Free IM Providers and Clients for
Your Library". Public Services Quarterly, 2009, vol. 5, n 1, p. 41-48.

Jacobson Terra B. Facebook as a Library Tool: Perceived v. Actual Use. [Preprint]. Accepté le 2 février 2010. Association of College and Research Libraries. [En ligne]

< http://www.ala.org/ala/mgrps/divs/ acrl/publications/crljournal/preprints/ crl-088.pdf > (consulté le 12 avril 2010).

Jackson Catherine. « Le service de réponses à distance de l'enssib : tout sur les bibliothèques ». Bulletin des bibliothèques de France, 2009, t. 54, $\mathrm{n}^{\circ}$ 4, p. 65-68. [En ligne]

< http://bbf.enssib.fr >

Jackson Catherine, Peyrelong MarieFrance. Le service « Questions ? Réponses ! » de l'enssib : un véritable processus d'échange. DocumentalisteSciences de l'information, vol. $46, \mathrm{n}^{\circ} 3$, septembre 2009, p. 66-67.

Kern Kathleen M. Virtual reference best practices tailoring services to your library. Chicago, American Library Association, 2009.

Koenig Marie-Hélène (dir.). Connaître les publics : savoir pour agir. Villeurbanne, Institut de formation des bibliothécaires, 1998. (Collection La Boîte à outils ; 8). 
Lankes David et al. The Virtual Reference Experience: Integrating Theory into Practice. New York, NealSchuman publishers, 2004.

Lankes David, Nicholson Scott, Radford Marie L. Virtual_reference service: from competencies to assessment. New York, Neal-Schuman Publishers, 2008.

Liu Jia. The evaluation of worldwide digital reference services in libraries. Oxford, Chandos, 2007. (Chandos information professional series).

Lupien Pascal, Rourke Lorna Evelyn. "Out of the Question!... How We Are Using Our Students” Virtual Reference Questions to Add a Personal Touch to a Virtual World. [En ligne] [s.l.] [s.n.], 2007

< http://ejournals.library.ualberta.ca/ index.php/EBLIP/article/viewArticle/ 236 > (consulté le 30 mars 2010).

Mc Lure Charles R. et al. Statistics, Measures, and Quality Standards for Assessing Digital Reference Library Services: Guidelines and Procedures. Syracuse: Information Institute of Syracuse (Syracuse University), School of Information Studies (Florida State University), 2002.

Miribel Marielle (de). " Les outils du bibliothécaire de référence » in Accueillir les publics, comprendre pour agir. Paris, Éditions du Cercle de la librairie, 2009, p. 407-442.
Nilsen Kirsti. «Services de référence en ligne contre service de référence sur place : une comparaison des pratiques de service de référence dans les bibliothèques publiques et académiques ». $71^{e}$ congrès de l'IFLA, 14 août 2005, Oslo. [En ligne] < http://www.ifla.org/IV/ifla71/papers/ 027e-Nilsen.pdf > (consulté le 12 avril 2010).

Nguyen Claire. "Les services de référence virtuels en bibliothèque universitaire ». Bulletin des bibliothèques de France, 2006, t. 51, n 3, p. 54-57. [En ligne] < http://bbf.enssib.fr >

Nguyen Claire. Mettre en place et développer un service de référence virtuelle dans une perspective d'intégration à un réseau collaboratif. Analyses comparées et propositions à partir de l'exemple de l'Université de Montréal. Mémoire d'étude. Diplôme de conservateur de bibliothèque. Villeurbanne, enssib, 2006, 2 volumes.

Primary Research Group. Creating the Virtual Reference Service. New York, Primary research Group, 2003.

Profit Steven K. A "History of the Developments in Virtual Reference Services at the College of Charleston Library”. Internet Reference Services Quarterly, 2009, vol. 14, n 1, p. 3-12. 
Ranjard Sophie. "Évaluer la demande et les besoins en informations ». Documentaliste-Sciences de l'information, volume $38, \mathrm{n}^{\circ} 1$, mars 2001, p. 1423.

\section{Ressources humaines et développe- ment Canada ; bureau des technolo- gies d'apprentissage (BTA). « Initiation aux principes fondamentaux de la gestion de projet » in Ressources humaines et développement des compé- tences Canada. 2003. [En ligne] < http://www.hrsdc.gc.ca/fra/pip/daa/ bta/ressources/boite_outils/gestion_de_ projet/intro_gestion_de_projet.shtml > (consulté le 12 avril 2010).}

Reginelli Marie. Archivage et publication en ligne des questions/réponses d'un service de référence à distance : étude d'opportunité d'un blogue pour SwissInfoDesk. Neufchâtel, CESID Diplôme universitaire de formation continue en information documentaire. Université de Genève, Faculté des Lettres. 30 p. Mémoire. 2009 (non publié).

Rowley Jennifer. "Managing branding and corporate image for library and information services". Library Review, 1997, vol. 46, n 4, p. 244-250.

Shachaf Pnina. "The paradox of expertise: is the Wikipedia Reference Desk as good as your Library?". Journal of Documentation, 2009, vol. 65, $\mathrm{n}^{\circ} 6$, p. 977-996.
Soret David. « Les services de référence dans un environnement concurrentiel ». Bulletin des bibliothèques de France, 2007, t. 52, n 6, p. 20-24. [En ligne] < http://bbf.enssib.fr >

Stahr Beth. "SMS library reference service options”. Library Hi Tech News, 2009, vol. 26, n³/4, p. 13-15.

Theiss-White Danielle. et al. "IM'ing overload: Libraryh3lp to the rescue”. Library Hi Tech News, 2009, vol. 26, $\mathrm{n}^{\circ} 1 / 2$, p. 12-17.

Vernette Éric. Techniques d'études de marché : savoir conduire toutes les étapes d'une étude de marché. Paris, Vuibert, 2000.

Verry-Jolivet Corinne (dir.). Créer et gérer un service de référence. Villeurbanne, Institut de formation des bibliothécaires, 1996 (Collection La Boîte à outils ; 3). 


\section{LISTE DES AUTEURS}

\author{
Jean-Philippe Accart \\ Directeur \\ Bibliothèque de la faculté des sciences \\ (Genève, Suisse)
}

\section{Nicolas Alarcon}

Responsable de la documentation

électronique et des enquêtes

Service commun de la documentation

de l'université d'Angers

\section{Caroline Bruley}

Responsable du SID et de

la bibliothèque de sciences

Service commun de la documentation de l'université Jean-Monnet (SaintÉtienne)

\section{Bertrand Calenge}

Responsable de l'Évaluation prospective

- communication interne

Relations avec les professionnels

Bibliothèque municipale de Lyon

\section{Isabelle Copin}

Coordinatrice des services à distance à

la direction des collections,

Bibliothèque nationale de France (Paris)

\section{Nathalie Daigne}

Service accueil des publics

Bibliothèque publique d'information

(Paris)

\author{
Marie-Françoise Defosse \\ Maître de conférences associée, \\ consultante en gestion de l'information, \\ enssib
}

\section{Christelle Di Pietro}

Chargée des projets de veille

Département des services

aux bibliothèques, enssib

\section{Hélène Gautier-Gentès}

Responsable du service de la rétroconversion

Bibliothèque interuniversitaire de médecine et d'odontologie (BIUM, Paris)

\section{Jean-Charles Houpier}

Responsable du secteur Santé Service commun de la documentation de l'université Henri-Poincaré (UHP) Nancy

\section{Nadine Kiker}

Directrice adjointe - responsable des formations et de la valorisation Service commun de la documentation de l'université d'Angers

\section{Sandrine Lebastard}

Coordinatrice de Questions-santé

Cité de la santé

Bibliothèque des sciences et

de l'industrie (BSI)

Universcience, Cité des sciences et de l'industrie (Paris) 


\section{François Michaud}

Directeur de la bibliothèque interuniversitaire Sainte-Barbe (Paris)

Coordinateur de Rue des Facs

\section{Pierre Moison}

Coordinateur de Questions ? Réponses ! Département des services aux bibliothèques, enssib

\section{Marie Montano}

Responsable du service aux publics Service commun de la documentation de l'université Paris Ouest La Défense

\section{Claire Nguyen}

Responsable de BIUMINFO et du service des périodiques et des bases de données Bibliothèque interuniversitaire de médecine et d'odontologie (BIUM, Paris)

\section{Tù-Tâm Nguyên}

Responsable de la Cité de la santé

Bibliothèque des sciences et de l'industrie (BSI)

Universcience, Cité des sciences et de l'industrie (Paris)

\section{Annie Prunet}

Responsable du service de références de la BMVR de Marseille

\section{Anna Svenbro}

Chargée de collections langues et littératures scandinaves Direction des collections Département littérature et arts Bibliothèque nationale de France (Paris)

\section{Hélène Tardif}

Bibliothécaire de référence

Bibliothèque ÉPC-Biologie

Direction des bibliothèques

Université de Montréal (Canada)
Secrétariat d'édition :

Silvia Ceccani

Mise en page :

Alexandre Bocquier

Conception graphique :

atelier Perluette, 69001 Lyon

www.perluette-atelier.com
Achevé d'imprimer

en juin 2010

par l'imprimerie Chirat

Dépôt légal :

$1^{\text {er }}$ semestre 2010 


\section{LA BOÎTE À OUTILS}

\section{METTRE EN GEUVRE UN SERVICE DE QUESTIONS-RÉPONSES EN LIGNE}

En quelques années, les bibliothèques françaises ont développé des services de questions-réponses en ligne. Beaucoup d'établissements en sont encore à la phase de réflexion, d'autres démarrent, des réseaux s'élaborent. Dans le contexte actuel du repérage, de la sélection et de la validation de l'information pléthorique disponible sur Internet, les services de questions-réponses en ligne ont un rôle primordial à jouer.

En effet, ils valorisent l'offre des bibliothèques dans le contexte du développement des services aux publics et des services à distance. Leur mise en place interroge le professionnel sous de multiples facettes : organisation du travail, politique de services, choix des outils... Ces différents aspects sont abordés, s'appuyant sur des expériences françaises et étrangères, qu'elles soient locales, coopératives et/ou collaboratives. Un éventail d'offres existe aujourd'hui, selon les objectifs, les besoins, les publics, les moyens mis à disposition et les technologies choisies.

Le plan s'articule autour de quatre parties pragmatiques et opératoires : construire le projet, développer un savoir-répondre, organiser et travailler au sein d'une équipe et, enfin, adapter le service à l'environnement et à la nature de ses publics.

Cet ouvrage collectif, coordonné par Claire Nguyen, se veut un guide utile à tous les professionnels qui veulent créer, maintenir ou faire évoluer un service de questions-réponses à distance. 